\title{
Growth Morphology and Properties of Metals on Graphene
}

Xiaojie Liua, Yong Han ${ }^{\mathrm{b}, \mathrm{c}}$, James W. Evans ${ }^{\mathrm{b}, \mathrm{c}}$, Albert K.Engstfeld ${ }^{\mathrm{d}}$, R. Juergen Behm ${ }^{\mathrm{d}}$, Michael C. Tringides ${ }^{\mathrm{b}, c}$, Myron Hupalo ${ }^{\mathrm{c}}$, Hai-Qing Lin ${ }^{\mathrm{e}}$, Li Huang ${ }^{\mathrm{f}}$, Kai-Ming Ho ${ }^{\mathrm{b}, \mathrm{c}}$, David Appy $^{\mathrm{c}, \mathrm{g}}$, Patricia A. Thiel ${ }^{\mathrm{c}, \mathrm{gh},}$, and Cai-Zhuang Wang ${ }^{\mathrm{c}}$

${ }^{a}$ Center for Quantum Science and School of Physics, Northeast Normal University, Changchun, 130117, China

${ }^{b}$ Department of Physics and Astronomy, Iowa State University, Ames, IA, 50011, U. S. A.

'Ames Laboratory - U. S. Department of Energy, Iowa State University, Ames, IA, 50011, U. S. A.

${ }^{\mathrm{d} I n s t i t u t e}$ of Surface Chemistry and Catalysis, Ulm University, D-89069, Ulm Germany

${ }^{\mathrm{e} B e i j i n g ~ C o m p u t a t i o n a l ~ S c i e n c e ~ R e s e a r c h ~ C e n t e r, ~ B e i j i n g, ~ 100084, ~ C h i n a ~}$

'Department of Physics, South University of Science and Technology of China, Shenzhen 518055, P. R. China

${ }^{8}$ Department of Chemistry, Iowa State University, Ames, IA, 50011, U. S. A.

${ }^{\mathrm{h}}$ Department of Materials Science and Engineering, Iowa State University, Ames, IA, 50011, U. S. A.

\begin{abstract}
Graphene, a single atomic layer of graphite, has been the focus of recent intensive studies due to its novel electronic and structural properties. Metals grown on graphene also have been of interest because of their potential use as metal contacts in graphene devices, for spintronics applications, and for catalysis. All of these applications require good understanding and control of the metal growth morphology, which in part reflects the strength of the metal-graphene bond. Also of importance is whether the interaction between graphene and metal is sufficiently strong to modify the electronic structure of graphene. In this review, we will discuss recent experimental and computational studies related to deposition of metals on graphene supported on various substrates $\left(\mathrm{SiC}, \mathrm{SiO}_{2}\right.$, and hexagonal close-packed metal surfaces). Of specific interest are the metal-graphene interactions (adsorption energies and diffusion barriers of metal adatoms), and the crystal structures and thermal stability of the metal nanoclusters.
\end{abstract}

\section{Key words:}

Graphene, metal adsorption on graphene, growth morphology, metal-graphene interaction, metal nanoclusters, thermal stability 


\section{Contents}

1. Introduction

2. Growth morphology for metals on SiC-supported graphene

2.1 Alkali metal (AM) growth on graphene

$2.23 d$ Transition metal (TM) growth on graphene

2.3 Rare-earth metal (REM) growth on graphene

2.4 Other metal growth on graphene

3. Metals on few-layer-graphene (FLG) on $\mathrm{SiO}_{2} / \mathrm{Si}$

3.1 Au on FLG/SiO

3.2 Ag on FLG/SiO

3.3 Other metals on FLG/SiO

4. Metal nanostructures on metal-supported monolayer graphene: Overview

4.1 Moiré structure of monolayer graphene supported on metal substrates

4.2 Characterization of metal nanocluster distributions for directed assembly on metal-supported graphene

5. Directed assembly of metal nanoclusters on metal-supported graphene: Experiment

5.1 Metals on graphene on $\operatorname{Ir}(111)$

5.2 Metals on graphene on $\operatorname{Ru}(0001)$

6. Directed assembly of metal nanoclusters on metal-supported graphene: Theory and Modeling

6.1 Atomistic modeling and KMC simulation of directed-assembly

6.2. Application of modeling to specific systems

6.3. DFT analysis of energetics for metals on metal-supported graphene

7. General trends in growth morphology and stability

7.1 Growth morphology

7.2. Thermal stability of metal NC

8. Electronic and magnetic properties

9. Metals on graphite: Comparison to graphene

9.1 General comments

9.2 Case study: $\mathrm{Cu}$ deposited on graphite

10. Intercalation for supported graphene systems and for graphite

10.1 Effect of intercalation on surface metal growth

10.2 Driving force and mechanism for intercalation and the intercalant distribution

11. Summary 


\section{Introduction}

Graphene and its novel properties have been studied extensively over the last decade [1-11]. Its unusual electronic structure holds the promise of potential applications in many areas, especially for the new generation of ultrafast microelectronics. Its unique properties are related to the linear energy dispersion, with the two characteristic Dirac cones touching at a single point, and also to high electron mobility. Although these properties of clean graphene have been confirmed by many experimental measurements and theoretical calculations, open questions remain regarding the interactions of foreign atoms with graphene, especially metal atoms.

There are numerous potential applications of graphene that require a good understanding of graphene-metal interactions. For example, a strong metal-graphene interaction is necessary to ensure high quality metal contacts for device applications, but the interaction should not be so strong as to destroy the essential features of the graphene electronic structure. Metals have been used as dopants to control the Fermi level $\mathrm{E}_{\mathrm{F}}$ via charge transfer [5-6], but again the transfer should not be so extensive as to disturb the electron density distribution and degrade the electronic properties of graphene. So far, most of the doping experiments have been carried out without systematic control of the arrangement or morphology of dopant atoms. These metal atoms could exist within the layers of a smooth film (Frank van der Merwe growth), within three-dimensional nanoclusters (NCs) (Volmer-Weber growth), or as isolated adatoms on graphene. Different metal/graphene interface morphologies can modify the graphene electronic structure in adsorbate specific manner. If magnetic metals are deposited on graphene, the scope of applications is even larger: the grown structures can be used as spin filters [7-8], as high magnetic anisotropy materials [8], or as systems exhibiting novel Kondo effects [9]. They can also be used to magnetize graphene itself [10], to grow high densities of magnetic nanoclusters for computer storage applications [8-10], or even to induce superconductivity in graphene [11-12].

The purpose of this review is to survey a variety of metal/graphene systems and draw some general conclusions about the interactions of metal with graphene. We primarily focus on studies in which the graphene synthesis and metal deposition both occur in ultrahigh vacuum (UHV). These systems are ideal models for understanding the fundamental aspects of the metal-graphene interaction. We first consider graphene layers that are formed by removal of near-surface Si from SiC. These are attractive substrates for growth because these graphene layers can exhibit extended, defect-free regions and they have weak interaction with the underlying material. Second, we consider another class of substrates where graphene is transferred to, or grown on, a $\mathrm{SiO}_{2}$ film which is in turn supported on Si. Finally, we consider another intriguing route to in situ formation of graphene which involves chemical vapor deposition on extended metal surfaces, and we will survey this area as well.

Thermal annealing of Si-terminated SiC produces single layer (G1) or bilayer (G2) graphene of very high quality with domains that extend over several $\mu \mathrm{m}$ by the slow and controlled desorption of $\mathrm{Si}$ from steps on the $\mathrm{SiC}(0001)$ surface. The fraction of graphene in the form of $\mathrm{G} 1$ and $\mathrm{G} 2$ can be controlled mainly by adjusting the annealing rate to reach the graphitization temperature of $\sim 1470 \mathrm{~K}$ with a faster rate favoring G1 over G2 [13-15]. The graphene lattice, showing corrugation attributable to a $6 \sqrt{ } 3$ reconstruction of a carbon-rich buffer layer between graphene and $\mathrm{SiC}$ substrate, is presented in a high-resolution STM image in Fig.1. A number of experimental studies of the growth morphology of metals on graphene on Si-terminated SiC(0001) substrates have been 
reported recently, and these will be reviewed below. It has been shown that deposition of different metals on graphene can exhibit different growth morphologies and stability due to the different nature and strength of interaction between the metals and graphene. Motivated by the experimental studies, theoretical calculations based on first-principles density functional theory (DFT) for freestanding or unsupported graphene (noting that the graphene-SiC interaction is weak) have also been performed to investigate the metal-graphene interaction and changes in the graphene properties emerging after metal adsorption. These experimental and theoretical studies will be reviewed in this paper.

Another class of systems receiving recent attention involves deposition of metals on few-layer graphene (FLG) supported on $\mathrm{SiO}_{2}$ thin films. These studies often emphasize the influence of electrostatic interactions in controlling the distribution and morphology of metal films and nanoclusters [16-34]. We will briefly review observed behavior in these systems.

We also note that the rumpled structure of graphene supported on many single-crystal transition metal surfaces, which is due to lattice mismatch, provides a natural, periodically-templated substrate [35] with the potential to direct self-assembly of metal NCs. (In contrast, the stochastic nature of homogeneous nucleation on uniform flat SiC-supported graphene sheets limits the control of NC locations.) The resulting periodically ordered arrays of metal NCs provide model systems for fundamental studies of catalysis and plasmonics. For example, a well-defined periodic moiré pattern is formed between graphene and the metal substrate on $\mathrm{Cu}(111)$ [36-37], but not on $\mathrm{Ni}(111)$ [38-40]. A prominent moiré pattern also occurs for $\mathrm{Au}(111)$ [41], $\mathrm{Ru}(0001)$ [42-48], $\operatorname{Ir}(111)$ [49-51], and various other substrates. The reason for directed assembly lies in the feature that the graphene-substrate interaction is strong, and as a result the energetics for adsorbed metal adatoms exhibit significant periodic variation across the moiré supercell. This feature can induce specific preferred nucleation sites within the supercell, so that deposited atoms may aggregate at these locations, forming a regular periodic NC array. In addition, a high barrier for diffusion between moire cells enables the formation of a periodic array of small stable NCs. There have been many studies with STM of metal NC formation for graphene supported on the above metal surfaces, and these will be described later in some detail in this review. We also describe related DFT analysis and atomistic-level modeling of directed-assembly.

The rest of this article is organized as follows. Experimental observations of the growth morphology of metals on SiC-supported graphene will be discussed in Section 2. The distinctive behavior of metals on $\mathrm{SiO}_{2}$-supported graphene, where electrostatic interactions often dominate, is reported in Section 3. In Section 4, we provide an overview of directed-assembly of metal nanoclusters on metal-supported G1.We discuss both the rumpled structure of graphene sheets guiding directed-assembly, and the relevant quantities for characterization of NC distributions. A detailed description of experimental observations for metal NCs on metal-supported graphene is provided in Section 5, focusing on $\operatorname{Ir}(111)$ and $\operatorname{Ru}(0001)$ substrates. Then, atomistic-level modeling of directed-assembly in these systems and related DFT analysis is described in Section 6. This is followed by a general discussion of factors impacting the growth morphology and thermal stability in Section 7, and a discussion of the electronic and magnetic properties of metal NCs in Sections 8. Comparisons between adsorption of metals on graphene and on graphite are drawn in Section 9. Intercalation for supported graphene systems and for graphite is in Section 10. Finally, a summary will be given in Section 11. 


\section{Growth Morphology for metals on SiC-supported graphene}

Single layer (G1) or bilayer (G2) graphene produced by thermal annealing of Si-terminated SiCis almost defect free and has very weak interaction with the substrate. Therefore, metal deposition and growth on SiC-supported graphene provides an excellent model system for studying and understanding the interaction between metals and graphene. In this section, the experimental deposition studies were performed at room temperature (RT) unless otherwise noted.

\subsection{Alkali metal (AM) growth on graphene}

Alkali metals on graphene are of great interest and importance for potential device applications because the carrier density in graphene can be controlled by alkali metal doping while keeping the characteristic band structure of graphene intact. The growth morphology of several alkali metals on graphene, including Li [52], Na [53], as well as Cs [54] has been studied experimentally. It was shown [52] that Li deposition on graphene supported by $\mathrm{SiC}(0001)$ at room temperature can form islands or droplets, as shown in Fig. 2(a). The lateral size of the islands shown in Fig. 2 (a) is about $70 \mathrm{~nm}$. In comparison, a linear chain structure [53] is found for Na on graphene at a low coverage as shown in Fig. 2 (b). When the coverage increases, the $\mathrm{Na}$ chains become dense and coalesce into islands which are characterized by $6 \times 6$ corrugation structures, followed by intercalation of $\mathrm{Na}$ between the buffer layer and the graphene layer. We are not aware of any experimental studies of $\mathrm{K}$ on graphene, but deposition of $\mathrm{K}$ on the basal graphite surface at very low temperature of $11 \mathrm{~K}$ [55] has been reported. Fig. 2(c) shows that K adatoms on the graphite surface tend to form atomic superlattices. The formation of intriguing adatom superlattices is more clearly seen for Cs on graphene [54]. By using scanning tunneling microscopy (STM), it was shown that the formation of such Cs superlattices is dependent on the number of graphene layers and can be classified into three types: 1) short-range ordered hexagonal structures with an inter-adatom separation of $1.85 \mathrm{~nm}$ on monolayer graphene (i.e., Fig. 3 (a)); 2) long-range well-ordered superlattices on multilayer (less than six layers) graphene, with a periodicity of 1.85 and $3.20 \mathrm{~nm}$, corresponding to $1 \times 1$ and $\sqrt{3} \times \sqrt{3}$ Cs superlattices, respectively (i.e., Fig. 3 (b) and (c)); 3) disordered structures due to the collapse of the Cs superlattices (i.e., Fig. 3 (d)) when the number of graphene layers increases to six. It is also found that the density of Cs adatoms on bilayer graphene is substantially larger than that on monolayer graphene.

There have been extensive theoretical calculations devoted to the studies of adsorption energy [55-66] and diffusion barriers [56-60], as well as of properties such as charge transfer [56-62] and electric dipole moments [56-59] for alkali metals on graphene. From the first-principles calculations and bonding analysis [67], it has been shown that all alkali metal adatoms have their outer shell $s$ electron almost completely transferred to graphene, creating large electric dipole moments as shown in Table 1. The interaction between the alkali metal adatoms and graphene is strongly ionic as indicated by the small bond order parameters and relatively large adsorption energies shown in Table 1. It is interesting to note that although $\mathrm{K}$ and $\mathrm{Cs}$ adatoms on graphene have larger absorption energies, their diffusion barriers are very small ( $0.06 \mathrm{eV}$ for $\mathrm{K}$ and $0.03 \mathrm{eV}$ for $\mathrm{Cs})$. In comparison, $\mathrm{Li}$ and $\mathrm{Na}$ on graphene have higher diffusion barriers $(\sim 0.29 \mathrm{eV}$ for $\mathrm{Li}$ and $\sim 0.16 \mathrm{eV}$ for $\mathrm{Na})$. The diffusion barrier is anti-correlated with the size of the alkali adatom. The experimental observations of different growth morphologies for different alkali metals on graphene discussed above can be partially attributed to the different diffusion barriers. The larger 
diffusion barriers for $\mathrm{Li}$ and $\mathrm{Na}$ adatoms produce islands with smaller size while almost no barrier and large adsorption energy for $\mathrm{Cs}$ and $\mathrm{K}$ leads to the continuous growth of single atomic layers on graphene.

In addition to the interaction between an alkali adatom and graphene, the interaction between the adatoms should also play an important role in the growth morphology. The induced charge transfer and electric dipole moment will cause strong repulsions between the alkali adatoms due the dipole-dipole interaction and Coulomb interaction of the charged particles. Ref. 55 suggests that electric dipole-dipole interaction is the dominant interaction between $\mathrm{K}$ adatoms on a graphite surface. However, using first-principles calculations, Liu et al. [68] showed that the screened Coulomb interaction between $\mathrm{K}^{+}$ions is much stronger than the dipole-dipole interaction as shown in Fig. 4. Experiments by Song et al. [54] also suggest strong screened Coulomb repulsion between Cs adatoms on graphene. It seems that the strong Coulomb repulsion and the very small diffusion barrier are responsible for the formation of atomic superlattices for $\mathrm{Cs}$ on graphene and $\mathrm{K}$ on graphite. However, the dependence of the superlattice structure on the number of graphene layers observed for Cs on graphene is still not well understood. Whether the growth morphology of $\mathrm{K}$ on graphene will be different from that on graphite also is an open question. Moreover, $\mathrm{Li}$ and $\mathrm{Na}$ adatoms should also exhibit Coulomb repulsions due to charge transfer, but the strength of these interactions and how they influence the growth morphology of Li and $\mathrm{Na}$ on graphene still needs further investigation.

\subsection{3d Transition metals (TM) growth on graphene}

Due to their large local magnetic moment, there has been much interest in growing $3 d$ transition metal films, especially $\mathrm{Fe}$ and $\mathrm{Co}$, on graphene for potential spintronics device and magnetic storage applications. STM experiments [69-74] have shown that the growth of $3 d$ transition metals on graphene mainly exhibits a Volmer-Weber mode, i.e., formation of three-dimensional (3D) nanoclusters presumably close to equilibrium. The growth morphology of $\mathrm{Mn}, \mathrm{Fe}, \mathrm{Co}$, and $\mathrm{Ni}$ on graphene is shown in Fig. 5. The mean island size for $\mathrm{Fe}$ and $\mathrm{Co}$ on graphene is similar, while the island size for $\mathrm{Ni}$ is smaller and that of $\mathrm{Mn}$ is bigger (at the same coverage). The distribution of island sizes for $\mathrm{Ni}, \mathrm{Co}$, and $\mathrm{Fe}$ is quite narrow, while a substantial population of significantly larger-than-average islands is observed for Mn on graphene. First-principles calculations have been performed to study the adsorption energy and diffusion barriers of $3 d$ transition metals on graphene [9,75-77]. The adsorption energy $\mathrm{E}_{\mathrm{a}}$ increases from $\mathrm{Fe}$ to $\mathrm{Co}$ and then to $\mathrm{Ni}$ as one can see from Table 1. However, the diffusion barrier decreases from $\mathrm{Fe}$ to $\mathrm{Co}$ and then to $\mathrm{Ni}$. The adsorption energies and the bond order parameters of these three elements are large, indicating strong covalent bonding with graphene. By contrast, Mn exhibits much weaker interaction with graphene and has a very small diffusion barrier $(0.068 \mathrm{eV})$. There is a correlation between the experimentally observed growth morphology, and the calculated adsorption energy and diffusion barrier, which will be discussed in more detail later in section 7 .

The most interesting experimental observation for deposition of $\mathrm{Fe}$ and $\mathrm{Co}$ on graphene is the continuous increase of island density as the coverage $\theta$ increases [70-71]. For practically all metals grown on graphene, the coverage dependence of the nanocluster (NC) density $\mathrm{N}$ follows standard surface nucleation behavior, effectively saturating at low amounts of deposited material when the surface area covered is approximately 5\% [78]. However, for $\mathrm{Fe}$ on graphene, a steady nucleation of new islands can be seen in the 
persistent presence of small islands in Figs. 6(a) - (e). This behavior is observed as the coverage is varied over 3 orders of magnitude from 0.002 to $2.3 \mathrm{ML}$. We note that for very low coverage, indeed a very low $\mathrm{N}$ is observed, where these initial Fe islands are still 3D and contain several hundred atoms. This shows that initially the diffusion length is at least $50 \mathrm{~nm}$ (the average island separation in Fig. 6(a)), since the large number of deposited atoms incorporated in the islands must traverse such large distances. A continuous increase of the island density with deposition coverage is also observed for Co on graphene [71] as shown in Fig. 7.

The anomalous behavior of the island density for $\mathrm{Fe}$ and $\mathrm{Co}$ on graphene observed from experiments seems to indicate the presence of long-range repulsive interactions between the $\mathrm{Fe}$ and $\mathrm{Co}$ adatoms that suppress the aggregation of the $\mathrm{Fe}$ and Co adatoms to preexisting NCs. To gain insight into this interesting phenomenon observed by experiments, first-principles calculations have been carried out to study the interaction between a pair of adatoms on graphene [79]. The interaction between Fe-Fe adatoms is found to consist of a short-range attraction and longer-range repulsion as shown in Fig. 8. It has been suggested that the repulsive interactions for Fe adatoms at large distances can account for the unusual nucleation of Fe NCs on graphene discussed above [70,79]. This hypothesis might be verified by the comparison of the experimental images with kinetic Monte Carlo simulations modeling growth with long range interactions obtained from first-principles DFT calculations discussed above. Future simulations along this line are desirable. In Section 6, we will contrast behavior for Co and Fe deposition, as well as deposition of other transition metals, on $\mathrm{SiC}$-supported graphene with that for $\operatorname{Ir}(111)$-supported graphene [80-86].

\subsection{Rare-earth metal (REM) growth on graphene}

Rare-earth metals (REMs) have very unusual magnetic properties and occupy a unique position in light emission technologies. They are becoming increasingly important in developing cutting-edge high-tech products. Therefore, REMs on graphene is an interesting topic. Fig. 9 shows the growth morphology of several REMs on epitaxial graphene supported by $\mathrm{SiC}(0001)$. The STM images show a very high nucleated NC density for Gd [87-88], Dy [15,89], and almost smooth growth for Eu [90-91]. The most interesting feature are the fractal-like Gd three-dimensional (3D) NCs on graphene. Fig. 10 shows the results for Gd deposition at room temperature (RT) as a function of coverage. The fractal-like 3D islands are seen over a wide range of coverage, while the NC size increases with coverage, and the NC density $N$ is constant at approximately $1.2 \times 10^{-3}$ $\mathrm{NCs} / \mathrm{nm}^{2}$. The fractal shape of the NCs persists even after annealing to high temperature of $800 \mathrm{~K}$.

Room temperature (RT) deposition of Dy on graphene shows a high density of islands as seen in Fig. 11 (a). When Dy growth is carried out at higher temperature and larger deposited amounts, crystalline multi-height NCs formed. As seen in Fig. 11(b) the shapes of the NCs are mostly triangular for growth at $660 \mathrm{~K}$ after continuous deposition up to a total amount of $1.36 \mathrm{ML}$. There are approximately an equal number of 3D NCs pointing in opposite directions. These are multi height NCs with perfectly completed, flat tops extending all the way to steep side walls.

Hexagonal shapes are expected for the hcp Dy crystal since all the six growth planes constituting the sides of a 3D NC are equivalent. The triangular shapes observed for Dy suggest that the crystal is fcc(111) since for fcc crystals the six side growth planes are 
nonequivalent and have either (111) or (100) orientation. When Dy is deposited sequentially in small increments, the Dy NCs grow bigger with incomplete top layers. The stacked layers point in the same direction as expected for fcc ABCABC stacking while for hcp ABAB stacking the direction would alternate. Changes in the crystallography of the NCs can modify the magnetic properties of the Dy nanostructures on graphene, and therefore widens their potential applications.

Only Dy NCs show these triangular shapes among the magnetic metals [15,87-91] (i.e., $\mathrm{Fe}, \mathrm{Eu}$, and $\mathrm{Gd}$ ) on graphene studied to date. It is interesting to note that these Dy NCs not only have ideal triangular shapes but they are multi-height $\sim 10$ layers even when the total amount deposited is much lower at $\sim 1.36 \mathrm{ML}$. In most other fcc metal systems, where analogous deviation from equilibrium Wulff shapes have been observed and attributed to anisotropic corner rounding, the NCs were single layer NCs [92]. These basic observations confirm again not only the 3D growth mode for metals on graphene, but also the strong preference of Dy to be exclusively in the fcc crystal structure when grown on graphene. It would be interesting to clarify the role of graphene since bulk-like hcp(0001) Dy NCs grow on W(110) [93-94]. In another comparison, an earlier study using in situ resistivity and electron diffraction showed that Dy deposited on a glass substrate grows initially as an fcc crystal up to a thickness of $20 \mathrm{~nm}$, followed by a gradual structure change from fcc to hcp, at higher thicknesses [95].

In comparison, Eu deposited at RT forms large crystalline clusters, mainly of height $0.75 \mathrm{~nm}$ (about 2 atomic $\mathrm{Eu}(110)$ layers) as one can see from Fig. 9 (c). The film closes smoothly if the deposition of Eu continues. We will review other metals on graphene supported by metal substrates in later sections, but we would like to point out here that layer-by-layer growth at RT is also observed for Eu on graphene supported on $\operatorname{Ir}(111)$ as shown in Fig. 12(h)-(i) [91]. However, the growth morphology of Eu at low temperature is different from that at RT. Fig. 12(a)-(f) shows a sequence of STM images resolving growth morphologies of Eu on graphene with an Ir substrate at low temperature (35K) for increasing amount of deposited $\mathrm{Eu}$ [91]. Eu clusters arrays with short range order can be seen from the STM images, especially Eu dimer. The island density increases initially with the deposited amount (Fig. 12(a)-(c)) and then saturates (Fig. 12(d) and (e)). The island density slightly decreases and the island size grows bigger as the coverage increases further (Fig. 12(f)) due to cluster coalescence.

The growth morphology differences for the three REMs on graphene are consistent with the calculated diffusion barriers listed in Table 1. Gd shows a relatively high diffusion barrier of $0.31 \mathrm{eV}$, followed by that of Dy $(\sim 0.18 \mathrm{eV})$ and then by that of Eu $(\sim 0.13 \mathrm{eV})$. The high density fractal islands of Gd would be attributed to its high diffusion barrier. On the other hand, the low diffusion barrier for Eu would explain why the Eu crystalline islands grow quickly in an almost layer-by-layer fashion.

\subsection{Other metal growth on graphene}

In addition to the alkali, $3 d$ transition, and rare earth metals discussed above, growth of other metals including $\mathrm{Pb}$ and $\mathrm{Cu}$ on graphene on $\mathrm{SiC}$ has also been investigated [96-104] due to their potential applications in electronic devices and nanotechnology.

Fig. 13(a) shows that in a $100 \times 100 \mathrm{~nm}^{2}$ area only three large $\mathrm{Pb}$ islands are observed with growth conditions $T=78 \mathrm{~K}$ and $\theta=0.5 \mathrm{ML}$. The NCs have heights of eight, seven and six layers and regular face-centered cubic (fcc) (111) shapes. The growth of large crystalline $\mathrm{Pb}$ islands on graphene at low temperature is consistent with first principles 
calculations showing that there is almost no barrier for $\mathrm{Pb}$ adatom diffusion on graphene, as one can see from Table 1. Fig. 13(b) shows an STM image of the zero layer graphene (ZLG) after deposition of a $\mathrm{Cu}$ film of thickness $12 \AA$. The surface is completely covered by many large clusters, which are considered to consist of deposited $\mathrm{Cu}$ atoms. Each large cluster has a diameter and a height of $10-20 \mathrm{~nm}$ and $0.5-1.0 \mathrm{~nm}$, respectively. First-principles calculations show that a $\mathrm{Cu}$ adatom on graphene has a similar adsorption energy and diffusion barrier as $\mathrm{Pb}$, yet the growth morphology of the two metals are not quite the same. The origin of such difference is still not well understood.

In Section 5.1, we describe the behavior for deposition of various metals ( $\mathrm{Ir}, \mathrm{Pt}, \mathrm{W}, \mathrm{Re}$, $\mathrm{Fe}, \mathrm{Au}$ ) on $\operatorname{Ir}(111)$-supported graphene, where Ir, Pt and $\mathrm{W}$ form well-ordered NC arrays, but not $\mathrm{Fe}$ and $\mathrm{Au}[105-108]$.

\section{Metals on few-layer-graphene (FLG) on $\mathrm{SiO}_{2} / \mathrm{Si}$}

Monocrystalline graphitic thin films supported on $\mathrm{SiO}_{2}$ were discovered in 2004 [1]. These films are only a few atomic layers thick, and are therefore referred to as few-layer graphene (FLG). Here, we use $n$-layer to denote the thickness of a graphene film. They are stable under ambient conditions [1]. Since their original discovery, various different methods have been reported for transfer-free synthesis (including etch precipitation, conventional or plasma enhanced CVD, dual flame methods, ion implantation) of FLG films supported on $\mathrm{SiO}_{2}$ substrates [109-117], as well as for transfer onto a $\mathrm{SiO}_{2}$ substrate [1,118-121].In these experimental studies, the $\mathrm{SiO}_{2}$ substrate is often of hundreds of $\mathrm{nm}$ thick and is itself supported on a thick $\mathrm{Si}$ wafer. In the last five years, several experimental groups have deposited various metals on $\mathrm{FLG} / \mathrm{SiO}_{2} / \mathrm{Si}$ to form metal clusters or films, and then observed dewetting and coarsening behavior after annealing at different temperatures [16-34]. A significant feature observed in these studies is that the morphology of metal films deposited on the $\mathrm{FLG} / \mathrm{SiO}_{2}$ has a strong dependence on the layer number $n$. We caution, however, that these studies are often not performed under UHV conditions.

\subsection{Au on FLG/SiO}

Zhou et al. [17] used scanning electron microscopy (SEM) to observe the morphologies of $\mathrm{Au}$ films and nanoclusters (NCs) on FLG on $\mathrm{SiO}_{2} / \mathrm{Si}$. Vacuum deposition of $\mathrm{Au}$ on the substrate was performed under ambient conditions $(\sim 300 \mathrm{~K})$. SEM studies revealed distinct morphologies at a high coverage of $\mathrm{Au}$ for different thicknesses of FLG, but morphologies are similar for $n \geq 4$. After annealing at $\sim 1535 \mathrm{~K}$ in vacuum for $30 \mathrm{~s}$, Au NCs form on all $n$-layer graphene films. Morphologies, sizes, and densities $(N)$ of $\mathrm{Au}$ NCs significantly depend on $n$-see Fig.14. Assuming that NC density is controlled by irreversible nucleation and growth for $2 \mathrm{D}$ nanoclusters with a simple scaling relation $N \sim D^{-1 / 3}$ at fixed coverage, where $D \sim \exp \left[-E_{\mathrm{d}} /\left(k_{B} T\right)\right]$ is the surface diffusion coefficient, large differences, $\Delta E_{\mathrm{d}} \sim 0.2-0.6 \mathrm{eV}$, in $\mathrm{E}_{\mathrm{d}}$ were deduced between thicknesses $n$ and $n+1$ for $n=1-3$. However, this result is not consistent with the values $(\sim 0.005-0.025 \mathrm{eV})$ of diffusion barriers of Au on G1, G2, and G3 from DFT calculations [30]. This result suggests that it may be incorrect to apply nucleation theory to this experimental situation, especially given that the Au islands are not observed immediately after preparation, but rather after extreme thermal treatment which would normally result in extensive equilibration. However, further experimental studies of the deposition of $\mathrm{Au}$ 
films onto $n$-layer graphene without thermal annealing are also described in [22], also showing a clear $n$-dependence of film morphologies.

The difference between as-deposited and annealed morphologies of $\mathrm{Au} \mathrm{NCs}$ and films on $\mathrm{FLG} / \mathrm{SiO}_{2} / \mathrm{Si}$ has also been examined in other studies. SEM images of $\mathrm{Au}$ deposition on $n$-layer graphene at $\sim 300-400 \mathrm{~K}$ show different NC structures, which tend to be polygonal for lower coverage, but dendritic clusters, or dense films for higher coverage [27]-see Fig.15. Annealing at $\sim 975 \mathrm{~K}$ for $2 \mathrm{~h}$ leads to a predominance of 6 -sided polygonal Au NCs, while annealing at $\sim 1075 \mathrm{~K}$ for $2 \mathrm{~h}$ produces the coexistence of 6-sided and triangular Au NCs [26] See Fig.16. This behavior is similar to the observed epitaxial growth of micrometer-sized Au particles on graphite [32] where the interface is a $\{111\}$ Au facet supported on the $\{0001\}$ face of graphite.

The $n$-dependent morphologies, sizes, and densities of $\mathrm{Au} \mathrm{NCs}$ on $\mathrm{FLG} / \mathrm{SiO}_{2} / \mathrm{Si}$ were also observed by Luo et al. [16]. They deposited Au at $300 \mathrm{~K}$, and then annealed at $675 \mathrm{~K}$. As observed in Ref. 17, Au NCs form on the $n$-layer graphene films. Lou et al. fitted the average NC diameters to a power law $d_{\mathrm{av}}=A n^{v}$ with exponent $v \sim 0.33$ and prefactor $A \sim 6.46$ (in $\mathrm{nm}$ ), as illustrated in Fig.17. Previous experiments $[33,34]$ suggested a picture where Au NC morphology is impacted by $n$-dependent charge transfer between Au NCs and graphene. This motivated a model in which $n$-dependent electrostatic coulomb and dipole terms [55] were introduced into the total energy for the supported NC array. The model predicted a thermal-equilibrium average diameter $d_{\mathrm{av}}=$ $5.9 n^{1 / 3}$ (in $\mathrm{nm}$ ), in good agreement with the above experimental measurements. The feature of $n$-dependent charge transfer between metals and underlying graphene was also demonstrated in later experiments [19], where $\mathrm{Ag}$ deposition induced n-doping of graphene, while Au deposition induced p-doping.

From the above, it is clear that information about thermally-induced morphological evolution is important. From Fig.15, Au film morphologies are irregular or dense before annealing, and evolve by dewetting into separate Au NCs after heating. Lee et al. [29] discussed this dewetting behavior for $\mathrm{Au}$ on $\mathrm{FLG} / \mathrm{SiO}_{2}$ substrates compared with $\mathrm{SiO}_{2}$ and ST-cut quartz wafers. In their experiments, 1-nm-thick Au thin films were prepared by electron beam deposition. They found that for each type of substrate, the mean sizes of the Au NCs increase and the areal densities of the Au NCs decrease during annealing at $1175 \mathrm{~K}$, but that the rate of change depends on substrate. They attribute this dependence to different interfacial interactions between the $\mathrm{Au} \mathrm{NC}$ and the substrates.

Liu et al. compared nucleation kinetics of $\mathrm{Au}$ NCs on graphene for various underlying substrates, also exploring the dependence on layer number $n$ [25]. However, e-beam evaporation was performed at higher pressure of $5 \times 10^{-7}$ Torr rather than under UHV conditions. They employed mean-field nucleation theory to analyze Au NC densities on different substrates obtained from AFM images. From this analysis, the $\mathrm{Au}$ adatom diffusion constant for 1-layer graphene on $\mathrm{SiO}_{2}$ is $\sim 50$ times smaller than that for h-BN-supported graphene and on the order of 800 times smaller than that for graphite, while 2-layer graphene on $\mathrm{SiO}_{2}$ shows a Au adatom diffusion constant similar to 1-layer graphene on h-BN. They estimated the adatom diffusion barrier to be on the order of 100 $m \mathrm{eV}$. They propose two factors contributing to the observed lower diffusion constants on 1-layer graphene: local surface roughness, and homogeneous loss of dispersion/van der Waals electronic stability in multilayers. However, there are numerous assumptions in this analysis. NC density scaling relations for fixed areal coverage are used rather than 
for fixed total coverage. The effect of impurities on nucleation is ignored. It is noted that $\mathrm{Au} \mathrm{NCs}$ themselves can have significant mobility producing ramified aggregates via $\mathrm{NC}-\mathrm{NC}$ aggregation, and this feature is not included in the theory. One should also account for post-deposition evolution of the NC array. Finally, Raman spectroscopy in this study indicates little charge transfer between $\mathrm{Au}$ nanoparticles and graphene, in contrast to the previous experiments [33,34] and the model analysis [16] where a strong $n$-dependent charge transfer between metals and underlying graphene was assumed.

Line defects, e.g., grain boundaries (GBs), wrinkles, etc., are unavoidable in graphene, but identifying their structural details is difficult. Yu et al. [28] effectively visualized such defects by depositing $\mathrm{Au}$ on graphene supported $\mathrm{SiO}_{2} / \mathrm{Si}$. Their $\mathrm{SEM}$ images show single lines and double lines of $\mathrm{Au}$ NCs formed along GBs and wrinkles, respectively. Their theoretical calculations show that the distinct characteristic morphology of gold NCs arrays is due to distinct Au binding energies at different line defects, which are correlated to disrupting diffusion of NCs. This approach could be expected to further exploit the defect structures of other two-dimensional materials.

\subsection{Ag on FLG/SiO}

Similar to $\mathrm{Au} \mathrm{NCs}$ forming on a $\mathrm{FLG} / \mathrm{SiO}_{2}$ substrate as described above, the morphologies, sizes, and densities of $\mathrm{Ag}$ NCs formed by depositing $\mathrm{Ag}$ on $\mathrm{FLG} / \mathrm{SiO}_{2}$ substrate are also $n$-dependent. In Zhou et al.'s deposition experiments, the dependence of Ag morphologies on $n=1$ or 2 was studied at 300, 330, and $370 \mathrm{~K}$ [20]. Again, they attributed the $n$-dependence and temperature variation to the changes in surface properties and/or Ag [17] surface diffusion coefficient. Huang et al. also performed Ag deposition experiments, exploring dependence on $n=1-2$ by first depositing $\mathrm{Ag}$ at $300 \mathrm{~K}$ and maintaining a temperature of $370 \mathrm{~K}$ in a vacuum for 1 hour [24]. They proposed that only variations in the interactions between Ag NCs and graphene films are responsible for the $n$-dependence. They also further found that the size and density of $\mathrm{Ag} \mathrm{NCs}$ on suspended graphene is similar to those on graphene supported by $\mathrm{FLG} / \mathrm{SiO}_{2}$.

\subsection{Other metals on FLG/SiO}

Somers et al. [18] thermally evaporated thin layers of $\mathrm{Yb}(\sim 0.22 \mathrm{~nm}$ and $\sim 0.5 \mathrm{~nm})$ and $\mathrm{Au}(\sim 0.3 \mathrm{~nm})$ onto the $\mathrm{FLG} / \mathrm{SiO}_{2} / \mathrm{Si}$ surfaces. Then, they annealed the $\mathrm{Yb}$ - and Au-deposited systems at $\sim 870$ and $\sim 670 \mathrm{~K}$, respectively, for 3 hours in a reducing atmosphere, 1:1 $\mathrm{H}_{2}$ and Ar. From the SEM images after annealing, the morphologies of $\mathrm{Au}$ are consistent with those in their previous experiments [16], i.e., nearly isotropic $\mathrm{Au}$ $\mathrm{NCs}$ form, and the average radius of $\mathrm{Au} \mathrm{NCs}$ increases with increasing $n$. However, in the SEM images of the morphologies of $\mathrm{Yb}$ on $\mathrm{FLG} / \mathrm{SiO}_{2} / \mathrm{Si}$, they found filamentary branched structures of $\mathrm{Yb}$ (which they described as labyrinthine), see Fig.18. They attributed the formation of this NC Yb structure to a combination of a significantly larger work-function mismatch with the graphene and a lower intrinsic surface tension, relative to the corresponding Au system. They developed a theoretical model for the associated shape instability during NC growth in terms of the electrostatic energy of the aggregates (treated as 2D structures with fixed height) which included renormalization of the line tension aggregate by intra-aggregate repulsive electrostatic interactions. Model analysis revealed a dominant quadrupolar growth mode instability elongating a circular droplet 
and producing a characteristic length consistent with the observed filamentary $\mathrm{Yb}$ structures.

Using deposition conditions similar to those for $\mathrm{Au}[16,17]$, Zhou et al. deposited various coverages of $\mathrm{Pd}$ onto the $\mathrm{FLG} / \mathrm{SiO}_{2}$ substrate surface [23]. An $n$-dependence of the Pd morphologies was observed similar to that for $\mathrm{Au}$ deposition [16,22]: With increasing $n$, the Pd morphologies become much coarser, and the characteristic NC size becomes much larger. Differences in morphologies disappear when $n \geqslant 4$. They also noted that $\mathrm{Pd}$ atoms more easily nucleate and grow along the graphene edges. Using Raman spectroscopy, they reported $n$-dependent charge transfer between $\mathrm{Pd}$ and the graphene layers. For additional discussion about charge transfer for $\mathrm{Au} / \mathrm{FLG} / \mathrm{SiO}_{2}$, also see $[16,18,25,33,34]$.

In similar experiments to those for $\mathrm{Au}[16,20]$, Zhou et al. [21] thermally evaporated various metals ( $\mathrm{Fe}, \mathrm{Co}, \mathrm{Ni}, \mathrm{Mn}, \mathrm{Al}, \mathrm{Pd}, \mathrm{Au}$, and $\mathrm{Ag}$ ) onto $n$-layer graphene on $\mathrm{SiO}_{2} / \mathrm{Si}$ substrate and on graphite. They assessed differences in aggregation behavior between ferromagnetic metals ( $\mathrm{Fe}, \mathrm{Co}$, and $\mathrm{Ni}$ ), paramagnetic metals ( $\mathrm{Mn}, \mathrm{Al}$, and $\mathrm{Pd}$ ), and diamagnetic metals ( $\mathrm{Au}$ and $\mathrm{Ag}$ ) without and with an external magnetic field during the evaporation of metals. For ferromagnetic and paramagnetic metals, a metal nanowire formed along the edge of the graphene sheet regardless of whether the edge had a zigzag or armchair structure. An adjacent nanogap separated this nanowire from the continuous metal film on the interior of the graphene sheet. Such nanowires were not found for diamagnetic metals. With an external magnetic field applied during evaporation, the above features for ferromagnetic and paramagnetic metals disappear, and the morphologies of diamagnetic metals do not change.

\section{Metal nanostructures on metal-supported monolayer graphene: Overview}

Deposition of metals on well-defined substrates (e.g., low-index surfaces of crystalline metals, semiconductors, oxides, graphite, etc.) offers a route for self-assembly of metal NCs mediated by surface diffusion [76,122]. For homogeneous nucleation on unstructured substrates, this approach leads to a monomodal NC size distribution with large standard deviation proportional to the average NC size. Distinct size distributions arise for heterogeneous nucleation which is controlled in part by the defect distributions. A lack of precise control of the size distributions is related to a lack of control of the spatial location of the NCs, as is evident from examples shown in this article for deposition on $\mathrm{SiC}$-supported and $\mathrm{SiO}_{2}$-supported graphene discussed in Sections 2 and 3. Directed assembly (DA) on periodically templated substrates provides one strategy to achieve better control of both spatial and size distributions. Here, nucleation is favored at specific locations in the unit cell of the template (for reasons discussed below), leading to roughly periodic arrays of NCs for which all NCs have a common local environment. Potentially, this can lead to much narrower size distributions, especially for large mean island sizes. This level of control of size and local environment also facilitates analysis of the properties of functional metal NCs, e.g., for catalysis or plasmonics applications.

An observation of central importance here is that a single (mono-) layer of graphene (G1) supported on transition metal substrates generally displays a periodically modulated moiré structure due to lattice mismatch with the substrate [35,123]. Such substrates of most interest in this report are $\operatorname{Ir}(111)$ [35] and $\mathrm{Ru}(0001)$ [35], but other possibilities include $\operatorname{Rh}(111)$ [35,124], $\operatorname{Pt}(111)$ [35,125], $\operatorname{Pd}(111)$ [35,126], $\operatorname{Re}(0001)$ [35,127], and $\mathrm{Cu}(111)$ [35]. In contrast, there is no significant moiré structure for G1 on $\mathrm{Ni}(111)$ [35] 
or $\mathrm{Co}(0001)[35,128]$ due to a good lattice match. Modulation of supported G1 (see Fig. 19) offers the possibility for the type of DA of metal NCs described above. Demonstration of the DA strategy for metal NCs on metal-supported G1 was first provided for the deposition of $\operatorname{Ir}$ on G1/Ir(111) [49], and subsequently for other metals on G1/Ir(111) [106]. DA has also been achieved for deposition of Pt [129-132], Ru [133-135], and $\mathrm{Rh}$ [132] on $\mathrm{G} 1 / \mathrm{Ru}(0001)$. In addition, DA has been observed for deposition of $\mathrm{Ni}$ on $\mathrm{G} 1 / \mathrm{Rh}(111)$ [124].

In the following subsections, we first describe the structure of periodically-modulated G1 on metal supports, focusing on $\mathrm{Ru}(0001)$ and $\operatorname{Ir}(111)$. This characterization is an essential prerequisite for the detailed interpretation and modeling of the DA of metal NCs. Next, we briefly review various quantities which are assessed to provide a precise characterization of these complex systems. Then, we present experimental observations for DA of mainly single-component metal NCs in Sec.5, followed by a discussion of atomistic-level modeling of these processes in Sec.6.

\subsection{Moiré structure of monolayer graphene supported on metal substrates}

One of the most extensively studied systems is $\mathrm{G} 1 / \mathrm{Ru}(0001)$ where typically a single rotational domain of G1 is observed. Structural models, which are based on input from both experiment and DFT analysis, have provided a detailed characterization of the graphene moiré structure. The experimentally determined area of the rhombic moiré cell is $A_{M} \approx 7.794 \mathrm{~nm}^{2}$ [42]. A commonly employed structure model compatible with this $\mathrm{A}_{\mathrm{M}}$-value, and which we adopt for our atomistic simulations, corresponds to a $(12 \times 12) \mathrm{C} /(11 \times 11) \mathrm{Ru}$ moiré cell $[42,44,131-132,136]$. The area of the $\mathrm{Ru}(0001)$ surface

unit cell is given by $A_{R u}=0.0634 \mathrm{~nm}^{2}\left(a_{R u}=0.2706, b_{R u}=0.428 \mathrm{~nm}\right)$. Thus, the area of the moiré cell in units of $A_{R u}$ or of $R u$ surface atoms satisfies $A^{*}{ }_{M}=A_{M} / A_{R u}=122.9$ which is in reasonable agreement with the value for the above structure model of $A^{*}{ }_{M}=121$. The moiré cell for $\mathrm{G} 1 / \mathrm{Ru}(0001)$ is a rhombus composed of an adjacent pair of equal-sized upright and inverted equilateral triangles with a side length of $\mathrm{L}_{\mathrm{M}} \approx 2.98 \mathrm{~nm}$ [42,44,131-132,136]. Three distinct regions in this rhombic moiré cell are characterized as follows: atop regions (the vertices of triangles) where $\mathrm{C}$-atom rings surround $\mathrm{Ru}$ surface atoms; and 'hcp' and 'fcc' regions (the centers of inverted and upright triangles, respectively) where $\mathrm{C}$-atom rings surround the hcp and fcc hollow sites on $\mathrm{Ru}(0001)$. See Fig. 19. The atop locations are higher than the hcp and fcc regions by $\approx 0.15 \mathrm{~nm}$ [49]. Note that there are also other reasonable choices for a $\mathrm{G} 1 / \mathrm{Ru}(0001)$ structure model including $(11 \times 11) \mathrm{C} /(10 \times 10) \mathrm{Ru}$ and $(23 \times 23) \mathrm{C} /(25 \times 25) \mathrm{Ru}[43,46,48,136]$.

Next, we briefly discuss the behavior of G1/Ir(111) [49,106]. Again, there is a well-characterized graphene moiré structure with a moiré cell area corresponding to $\mathrm{A}^{*}{ }_{\mathrm{M}}$ $\approx 87 \pm 3$ Ir atoms. A commonly employed structure model reasonably compatible with this $\mathrm{A}_{\mathrm{M}}$-value corresponds to a $(10 \times 10) \mathrm{C} /(9 \times 9) \operatorname{Ir}(111)$ moiré cell. (Note that the Ir surface lattice constant is $\mathrm{a}_{\mathrm{Ir}}=0.272 \mathrm{~nm}$ and the carbon repeat distance is $0.245 \mathrm{~nm}$.) The observed periodicity actually corresponds to $(9.32 \times 9.32) \operatorname{Ir}(111)$. Again the rhombic moiré cell for $\mathrm{G} 1 / \mathrm{Ir}(111)$ is composed of an adjacent pair of equal-sized upright and inverted equilateral triangles, now with a side length of $\mathrm{L}_{\mathrm{M}} \approx 2.52 \mathrm{~nm}$; there are three distinct regions which can be described as for $\mathrm{G} 1 / \mathrm{Ru}(0001)$, and which differ strongly in their ability to bind metal adatoms. Finally, we mention that $(12 \times 12) \mathrm{C} /(11 \times 11) \mathrm{Rh}(111)$ and $(10 \times 10) \mathrm{C} /(9 \times 9) \operatorname{Re}(0001)$ structure models have also been proposed, and that in contrast 
to $\mathrm{G} 1$ on $\mathrm{Ru}(0001)$, multiple rotational domains are observed for $\mathrm{G} 1$ on $\operatorname{Ir}(111), \operatorname{Pt}(111)$, $\mathrm{Pd}(111)$, and $\mathrm{Cu}(111)$ [137].

\subsection{Characterization of metal nanocluster distributions for directed assembly on metal-supported graphene}

We will use the notation of our previous studies [134,135]. We specify the coverage ( ) of the evaporated metal in monolayers (ML) with respect to the supporting metal surface. Then, one has that $\theta=\mathrm{Ft}$, where $\mathrm{F}$ is the deposition flux and the deposition time. NCs are defined as metal clusters with 2 or more adatoms. We note that under experimental conditions for the systems of interest here, at most one $\mathrm{NC}$ occupies each graphene moiré cell. Thus, it is convenient to describe the NC density in terms of the fraction of occupied moiré cells, a so-called filling factor (FF) [35,48,50,134]. Sometimes $\mathrm{FF}=\mathrm{FF}(\theta)$ is quoted as a percentage of occupied cells (rather than as a fraction). Also of interest is the mean NC size, $<\mathrm{s}>=<\mathrm{s}>(\theta)$, measured in atoms, a quantity which is not independent of the NC density. Rather, one has that $<\mathrm{s}>=\mathrm{A}^{*}{ }_{\mathrm{M}} \cdot\left(\theta-\theta_{1}\right) / \mathrm{FF}(\theta)$, where $\mathrm{FF}$ is measured as a fraction, $\theta$ is the total coverage, and $\theta_{1}$ is the coverage of isolated diffusing metal adatoms which are not yet incorporated into NCs (both coverages being measured in ML, as described above). Since typically $\theta_{1}<\theta$ for high adatom diffusivity (except for very low $\theta$ ), one obtains the simple relation $<\mathrm{s}>\approx \mathrm{A}^{*}{ }_{\mathrm{M}} \cdot \theta / \mathrm{FF}(\theta)$.

Nanocluster size and height distributions. It is also instructive to consider the normalized probability distribution, $\mathrm{P}_{\mathrm{s}}$, for finding $\mathrm{s}$ atoms in a moiré cell, where $\mathrm{s}=0$ corresponds to empty cells. Multiple atoms in a single cell are generally aggregated into a $\mathrm{NC}$, so $\mathrm{P}_{\mathrm{s} \geq 2}$ corresponds to the NC size distribution. Then, it follows that $\sum_{\mathrm{s} \geq_{0}} \mathrm{P}_{\mathrm{s}}=1, \sum_{\mathrm{s} \geq 2}$ $\mathrm{P}_{\mathrm{s}}=\mathrm{FF}$. Also if $<\mathrm{s}>_{\mathrm{j}}=\left(\sum_{\mathrm{s}} z_{\mathrm{j}} \mathrm{s} \mathrm{P}_{\mathrm{s}}\right) /\left(\sum_{\mathrm{s}}>_{\mathrm{j}} \mathrm{P}_{\mathrm{s}}\right)$, then it follows that $\langle\mathrm{s}\rangle_{0}=\mathrm{A}^{*}{ }_{\mathrm{M}} \theta$ gives the mean $\mathrm{NC}$ size also counting isolated adatoms and $\langle\mathrm{s}\rangle=\langle\mathrm{s}\rangle_{2}$ corresponds to the above standard definition of mean size for NCs with $\mathrm{s} \geq 2$. Typically for NC formation, one looks for scaling of the NC size distribution of the form $P_{s} \propto f(s /<s>)$ [78]. However, a key benchmark case without this scaling corresponds to the situation where there is no transport between cells. Then, $\mathrm{P}_{\mathrm{s}}$ is given by a Poisson distribution,

$\mathrm{P}_{\text {pois }}(\mathrm{s})=(\mathrm{s} !)^{-1}\left(<\mathrm{s}>_{0}\right)^{\mathrm{s}} \exp \left(-<\mathrm{s}>_{0}\right)$,

where $<\mathrm{s}>=<\mathrm{s}>_{0}\left[1-\exp \left(-<\mathrm{s}>_{0}\right)\right]\left[1-\left(1+<\mathrm{s}>_{0}\right) \exp \left(-<\mathrm{s}>_{0}\right)\right]^{-1}$.

With regard to the NC structure, a rough assessment of the propensity for $2 \mathrm{D}$ versus 3D growth can be made by comparing isolated metal adatom-G1 binding with metal-metal binding [138] as will be described in Sec.7.1. In any case, 3D NCs are most often observed, and their height distributions are often measured experimentally. One convenient characterization of height distributions in the case where one can resolve a discrete number of atomic layers, $h=1,2,3, \ldots$ is to consider height-resolved filling factors, $\mathrm{FF}(\mathrm{h})$. Here, $\mathrm{FF}(\mathrm{h})$ denotes the fraction of moiré cells populated by NCs of height h. Thus, one has $\sum_{\mathrm{h}} \geq_{1} \mathrm{FF}(\mathrm{h})=\mathrm{FF}$.

Spatial correlations in nanocluster arrays. For systems where DA leads to a strongly spatial localization at a specific region within the moiré cells, one expects a complete roughly periodic array of NCs at sufficiently high coverage. However, for lower coverages with partially complete arrays of $\mathrm{NCs}$ with $\mathrm{FF}<1$, the $\mathrm{NC}$ spatial arrangement is random only if there is surface mass transport between surface moiré cells. In general, 
significant transport of adatoms between moiré cells leads to spatial correlations in the populated cells, e.g. inhibition of nearby NC pairs or anti-clustering might be expected based on traditional nucleation theories [91]. Interestingly, the approaches for assessment of these correlations are equivalent to those used for classic periodic lattice-gas models. Two types of quantities can be assessed to characterize these spatial correlations [134]. First, the populations of nearest-neighbor NC pairs (i.e., NC dimers), as well as linear, bent, and triangular NC trimers can be considered. These suitably defined populations are higher (lower) than values for a random distribution of $(\mathrm{FF})^{2}$ for dimers, and $(\mathrm{FF})^{3}$ for trimers, for clustering (anti-clustering) of NCs. One can also consider standard short-range order parameters for various separations which are positive (negative) for clustering (anti-clustering) [134].

\section{Directed assembly of metal nanoclusters on metal-supported graphene: Experiment}

\subsection{Metals on graphene on $\operatorname{Ir}(111)$}

Ir nanocluster arrays on single-layer graphene/Ir(111). Pioneering studies for the DA of Ir NC on G1/Ir(111) involved Ir deposition at $350 \mathrm{~K}$ [49]. NCs form exclusively in the hcp regions at 350K. See Fig. 20(a). The following distinct regimes of NC formation were observed:

(i) $\mathrm{NC}$ nucleation up to $\sim 0.05 \mathrm{ML}$, at which point FF reaches $\sim 100 \%$. See Fig. 21(a). It was proposed that there is negligible inter-cell transport during deposition. Then, the lack of monomers and dimers observed in STM images taken $\sim 15$ minutes after deposition must reflect significant inter-cell mobility of these adspecies during this post-deposition period. Consequently, it follows that the post-deposition FF satisfies

$$
\begin{aligned}
\mathrm{FF} & =1-\mathrm{P}_{\text {pois }}(0)-\mathrm{P}_{\text {pois }}(1)-\mathrm{P}_{\text {pois }}(2) \\
& =1-\left[1+<\mathrm{s}>_{0}+1 / 2\left(<\mathrm{s}>_{0}\right)^{2}\right] \exp \left(-<\mathrm{s}>_{0}\right) \text { with }<\mathrm{s}>_{0}=87 \theta,
\end{aligned}
$$

yielding a coverage dependence for the $\mathrm{NC}$ density distinct from conventional nucleation theory [78]. For $\theta=0.03 \mathrm{ML}$, this yields $\mathrm{FF}=0.48$, contrasting the experimental $\mathrm{FF} \approx$ 0.65 . However, there is some uncertainty in $\theta$. An alternative analysis uses the experimental $<\mathrm{s}>\approx 4.0$ for $\theta=0.03$ ML to determine $<\mathrm{s}>_{0} \approx 3.6$ yielding $\mathrm{FF} \approx 0.70$ which is just above the experimental value. (The latter overestimate could reflect the presence of some post-deposition inter-cell trimer mobility.) We also note that the experimental $<\mathrm{s}>$ actually decreases slightly as $\theta$ increases from 0.01 to $0.03 \mathrm{ML}$ due to a strong increase in $\mathrm{FF}$ with $\theta$.

(ii) $\mathrm{NC}$ growth for fixed $\mathrm{FF} \approx 1$ and perfect hexagonal arrays of NCs yields a linear increase in $\langle\mathrm{s}>\propto \theta$. See Fig. 21(a). The NC size distribution quickly approaches the Poisson form, $\mathrm{P}_{\text {pois }}(\mathrm{s})$, already by $<\mathrm{s}>\approx 9$. This behavior is consistent with the picture of no intercell transport during deposition. Note that $\mathrm{P}_{\text {pois }}(\mathrm{s})$ is narrow in the sense that its width scales like square root of $\langle\mathrm{s}\rangle$ (not like $<\mathrm{s}>$ itself), so the width of the scaled size distribution, $\mathrm{f}$, decreases like $<\mathrm{s}>^{-1 / 2}$. We also mention that growth of NCs was assumed to be irreversible, i.e., no adatom detachment. 
(iii) NC coalescence above $\sim 1.5 \mathrm{ML}$ producing NC extending over several cells, and where height-reduction is observed in concert with coalescence.

The above picture of no inter-cell transport during deposition implies a random final post-deposition NC distribution (where final NC locations match those of NCs with size 3 or more at the end of deposition). However, STM images for 0.03 ML suggest weak spatial anti-clustering: The probability $0.37 \pm 0.025$ of nearest neighbor NC dimer pairs at $\mathrm{FF}=0.66$ is slightly below the value of $(\mathrm{FF})^{2}=0.44$ for a random distribution. (The quoted $\pm 2 \sigma$ uncertainty comes from analysis of a finite sample of $230 \mathrm{NCs}$ assuming a binomial distribution for the number of dimers.) This suggests some limitations of the above Poisson-derived model.

Finally, we briefly describe other observations for this system: (a) NCs are only 1 layer high up to $<\mathrm{s}>=6$, and 1 and 2 layers high with roughly equal populations at $<\mathrm{s}>=$ 25 . The average height increases to 3.1 layers at $\langle\mathrm{s}\rangle=70$ reflecting a thermodynamic preference for 3D growth. (b) Significant population of fcc (as well as hcp) regions of the moiré cell is found at and below $160 \mathrm{~K}$.

Other metal nanocluster arrays and islands on single-layer graphene/Ir(111) [106]. Fig.22 provides a comparison of the experimentally observed morphologies for various metals (Ir, Pt, W, Re, Fe, and Au) deposited on G1/Ir(111). Pt and W NC arrays are almost as well ordered as those of Ir. See Fig.22(a)-(c). By an average height analysis, it is found that Pt clusters tend to grow flatter than Ir clusters. Also, W NC's adopt a crystal structure different from those of Pt. Re NC arrays and are significantly less ordered. See Fig.22(d). However, in all cases, the hcp region of the moiré cell is preferred. In contrast, for deposition of Fe (Fig.22(e)) Ni, or Au (Fig.22(f)) on G1/Ir(111), no NC superlattice is formed, but rather large unstructured clusters. This behavior is somewhat similar to that on SiC-supported graphene (see Sec.2.2). The variation in binding of these metals to graphene across the moiré cell is apparently too weak to trap adatoms in the moiré cells.

In Sec.2.2, we discussed in some detail the formation of 3D Co NCs on SiC-supported graphene. Large Co clusters with size as large as 2700 atoms have also been observed for Co on graphene supported by an $\operatorname{Ir}(111)$ substrate. Co growth on graphene supported by an $\operatorname{Ir}(111)$ substrate also yields nearly perfectly layer-by-layer growth by using of pulsed laser deposition techniques [8]. Experiment has shown that ordered Co cluster arrays can be developed by Pt or Ir seeding [79].

\subsection{Metals on graphene on $\mathrm{Ru}(0001)$}

$R u$ nanocluster arrays on single-layer graphene/Ru(0001). For the DA of Ru NCs during $\mathrm{Ru}$ deposition at $305 \mathrm{~K}$ on $\mathrm{G} 1 / \mathrm{Ru}(0001)$ [134-135], NCs form in the fcc region of the moiré cell in contrast to Ir on G1/Ir(111). See Fig. 20(b). Also, FF increases much more slowly only reaching FF $=0.48$ by $\theta=0.15$ ML. See Fig. 21(b). Consequently, it is clear that in this regime, $\mathrm{Ru}$ adatoms are not simply trapped within the moiré cell where they were deposited. Rather, there exists significant transport between moiré cells which is a key factor in determining the number, size, and spatial arrangement of NCs. For this system, there is a detailed analysis of not just the increase of FF and $<\mathrm{s}>$ with $\theta$, but also of correlations in the spatial arrangement of NCs. For the latter, there is clear evidence of anti-clustering from an analysis of the NC dimer and trimer populations as well the short-range order parameter [134]. For example, the population of nearest-neighbor NC dimer pairs at $\mathrm{FF}=0.48$ is slightly below that for a random distribution $(0.20$ versus 
0.23). Such behavior is expected for homogeneous nucleation and growth of NCs on unstructured substrates [78], but also applies here for DA. Evolution of the NC height distribution with $\theta$ has also been characterized and reflects a thermodynamic preference for 3D growth [134-135].

Finally, we mention a separate study of DA of Ru NCs involving deposition at $200 \mathrm{~K}$ on $\mathrm{G} 1 / \mathrm{Ru}(0001)$ [133]. Again a strong preference is found for the fcc region. This study suggested aggregation of NCs into denser groups at small scales, as well as alignment with the crystallographic directions of the substrate. However, no statistics for aggregation were presented. STM images suggest an increase in FF $\approx 0.05 \pm 0.02$ at 0.02 ML to $\mathrm{FF} \approx 0.25$ at $0.3 \mathrm{ML}$, with modest increases in the mean of both diameter and height distributions. It is not clear why this FF value is lower than that in the above study for deposition at higher $\mathrm{T}=309 \mathrm{~K}$.

Pt nanocluster arrays on single-layer graphene/Ru(0001). There are several studies of the DA of Pt NC which all reveal NC formation in the fcc region on the moiré cell [129-131,139-140]. One detailed study involving deposition at low temperatures around 140-180 K [130] reveals three different regimes as for $\operatorname{Ir} / \mathrm{G} 1 / \operatorname{Ir}(111)$ : (i) NC nucleation below $\sim 0.15 \mathrm{ML}$ where $\mathrm{FF}=0.53,0.73,0.97$, and 1 (and $<\mathrm{s}>=14,20,23$, and 30) for $\theta$ $=0.06,0.12,0.18,0.24 \mathrm{ML}$, respectively. (ii) $\mathrm{NC}$ growth for $0.15 \mathrm{ML}<\theta<0.5 \mathrm{ML}$ where almost all cells are occupied with NCs at near identical locations within the cell. The fraction of NCs with height above 1 layer increases steadily with $\theta$. (iii) $\mathrm{NC}$ coalescence regime for $\theta>0.5 \mathrm{ML}$ where clusters extend over multiple cells. Other studies explored the DA of Pt NCs by deposition at a higher temperature, $309 \mathrm{~K}$, for which typical NC arrays are shown in Fig. 20(c). This results in a much slower increase of FF with $\theta$ compared to lower-T deposition, noting that FF only reaches 0.26 by 0.15 ML [139-140]. See Fig. 21(c).

$R h$ nanocluster arrays on single-layer/Ru(0001). One study explored the DA of Rh $\mathrm{NCs}$ during $\mathrm{Rh}$ deposition at $295 \mathrm{~K}$ on G1/Ru(0001) [132]. See Fig. 20(d). NCs again form in the fcc region of the moiré cell. Also, FF increases much more slowly only reaching $\mathrm{FF}=0.24$ by $\theta=0.80 \mathrm{ML}$. See Fig. 21(d). This study also characterized the monomodal NC diameter distribution as a function of $\theta$, as well as the average cluster diameter and height versus $\theta$.

Other metal nanocluster arrays and islands on single-layer/Ru(0001). Deposition of $\mathrm{Pd}$ [132] and Co [132,141-142] on G1/Ru(0001) at $295 \mathrm{~K}$ results in NC formation. $\mathrm{Pd}$ NCs nucleate in fcc regions, but Co NCs form at both fcc and hep regions. In both cases, the density of NCs is much lower than for $\mathrm{Ru}, \mathrm{Pt}$, or $\mathrm{Rh}$, and the size of the NCs is correspondingly larger with their bases covering several moiré cells. This behavior is tied to the weaker bonding of $\mathrm{Pd}$ and $\mathrm{Co}$ to $\mathrm{G} 1 / \mathrm{Ru}(0001)$, than for the other metals [138]. Deposition of $\mathrm{Au}$ on $\mathrm{G} 1 / \mathrm{Ru}(0001)$ results in a single-layer 2D Au film rather than in 3D NCs [78,132]. Given the weak binding of Au to G1/Ru(0001), the formation and stability up to room temperature of this $2 \mathrm{D} \mathrm{Au}$ film (versus 3D islands) was unexpected (but see Ref.143). A better understanding of the physical reasons can lead to layer-by-layer metal growth in other cases.

\section{Directed assembly of metal nanoclusters on metal-supported graphene: Theory and modeling}




\subsection{Atomistic modeling and KMC simulation of directed-assembly}

Here, we describe recent atomistic-level modeling and simulation of the DA of metal NCs on metal-supported graphene [134,135,140]. First, we present a generic description of the energetics in these systems which provide input to the stochastic atomistic lattice-gas models. Specific DFT results for these systems will be described later, in Sec.6.3. We then describe the different possible origins of directed assembly.

System energetics. Atomistic modeling requires specification of the potential energy surface (PES) describing the lateral variation of the binding energy of a metal adatom to the graphene sheet, $\mathrm{E}_{\mathrm{b}}(\underline{\mathrm{x}})$, as a function of continuous lateral position, $\underline{\mathrm{x}}$, for an isolated adatom. See Fig. 23. There are well-defined local adsorption sites in each carbon ring with separation $\mathrm{a} \approx 0.25 \mathrm{~nm}$, so the PES includes a short-range highly oscillatory variation on a length scale $\sim$ a (solid curve in Fig. 23). There is also a coarse-scale modulation of the PES on the length scale of the moire cell $\mathrm{L}_{\mathrm{M}}$ which guides DA. We let $\mathrm{E}_{\mathrm{ads}}(\underline{\mathrm{x}})$ denote the smoothly, slowly varying function matching $\mathrm{E}_{\mathrm{b}}(\underline{\mathrm{x}})$ at the adsorption sites which we label by $i$. Thus, one has $\mathrm{E}_{\mathrm{b}}(\mathrm{i})=\mathrm{E}_{\mathrm{ads}}(\mathrm{i})$ (dashed green curve). Let $\mathrm{E}_{\mathrm{TS}}(\underline{\mathrm{x}})$ denote $\mathrm{a}$ smoothly varying function matching $\mathrm{E}_{\mathrm{b}}(\underline{\mathrm{x}})$ at the transition states for hopping between adjacent sites (dashed red curve). Thus, for the transition state for hopping between an initial state, $\mathrm{i}$, and a final state, $\mathrm{f}$, we adopt the reasonable linear interpolation formula, $\mathrm{E}_{\mathrm{TS}}(\mathrm{i} \rightarrow \mathrm{f}) \approx\left[\mathrm{E}_{\mathrm{TS}}(\underline{\mathrm{x}}=\mathrm{i})+\mathrm{E}_{\mathrm{TS}}(\underline{\mathrm{x}}=\mathrm{f})\right] / 2$. The key input to our modeling is the collection of activation barriers for diffusive hopping $\mathrm{i} \rightarrow \mathrm{f}$ which then satisfy

$$
\mathrm{E}_{\mathrm{act}}(\mathrm{i} \rightarrow \mathrm{f})=\mathrm{E}_{\mathrm{TS}}(\mathrm{i} \rightarrow \mathrm{f})-\mathrm{E}_{\mathrm{ads}}(\mathrm{i}) \approx\left[\mathrm{E}_{\mathrm{TS}}(\mathrm{i})+\mathrm{E}_{\mathrm{TS}}(\mathrm{f})\right] / 2-\mathrm{E}_{\mathrm{ads}}(\mathrm{i})
$$

Below, we discuss different possible forms for $\mathrm{E}_{\mathrm{ads}}(\underline{\mathrm{x}})$ and $\mathrm{E}_{\mathrm{TS}}(\underline{\mathrm{x}})$ which induce DA.

Stochastic atomistic lattice-gas modeling. This modeling includes: (i) random deposition at adsorption sites on the substrate; (ii) biased hopping between adjacent adsorption sites with activation barriers, $E_{a c t}(i \rightarrow f)$, and Arrhenius hop rates, $h(i \rightarrow f)=$ $v \exp \left[-\mathrm{E}_{\mathrm{act}}(\mathrm{i} \rightarrow \mathrm{f}) /\left(\mathrm{k}_{\mathrm{B}} \mathrm{T}\right)\right]$ for temperature $\mathrm{T}$ and Boltzmann constant $\mathrm{k}_{\mathrm{B}}$, and with a common prefactor $v=10^{13} / \mathrm{s}$. This form is consistent with detailed-balance and is controlled by the modulated PES as specified above; and (iii) irreversible nucleation and growth of NC. The model is readily adapted to treat deposition of a single type of atom or sequential (or simultaneous) deposition of two or more types. In addition, a "point island" model $[78,143]$ is utilized which tracks only NC size and composition, but not structure. Specifically, in this model, each NC occupies a single adsorption site, but carries a size label to indicate the number of atoms of various types within the NC. These point island models have proved particularly efficient and effective in elucidating the behavior of NC densities (corresponding here to the filling fraction, FF), and NC size distributions. Here, we utilize kinetic Monte Carlo (KMC) simulation to assess model predictions.

Thermodynamically (versus kinetically) directed assembly. The standard picture is that DA is driven by stronger binding of the adsorbate to the surface in the region of NC nucleation [134,137,144]. For irreversible NC formation, the nucleation rate scales like $\mathrm{h}$ $\mathrm{n}^{2}$ where $\mathrm{h}$ is the local diffusive hop rate, and $\mathrm{n} \sim \exp \left[-\mathrm{E}_{\mathrm{ads}} /\left(\mathrm{k}_{\mathrm{B}} \mathrm{T}\right)\right]$ is the local adatom density which is enhanced by lower $\mathrm{E}_{\mathrm{ads}}$ (i.e. stronger binding). We set $\mathrm{E}_{\mathrm{ads}}(\underline{\mathrm{x}})=\mathrm{E}_{\mathrm{p}}(\underline{\mathrm{x}})$, a periodically varying function with a minimum in the region of strongest binding which is expected to be: (i) fcc for $\mathrm{G} 1 / \mathrm{Ru}(0001)$ or (ii) hcp for $\mathrm{G} 1 / \operatorname{Ir}(111)$. $\mathrm{E}_{\mathrm{ads}}$ is higher (i.e., 
weaker binding) by $\Delta$ in the (i) hcp for case (i), or the fcc region for case (ii), by $\delta$ at the fcc-hcp boundary, and by $\delta+\delta^{*}$ at the atop regions. See Fig.23 corresponding to case (i).

Two simple scenarios for thermodynamically-directed assembly are: (a) $E_{a d s}=E_{p}(\underline{x})$ and $\mathrm{E}_{\mathrm{TS}}=\mathrm{E}_{\mathrm{d}}+\mathrm{E}_{\mathrm{p}}(\underline{\mathrm{x}})$ with constant, $\mathrm{E}_{\mathrm{d}}[134,139]$. This choice with

$$
E_{a c t}(i \rightarrow f)=E_{d}+\left[E_{p}(f)-E_{p}(i)\right] / 2
$$

produces strong DA since binding is much stronger in specific regions (enhancing $\mathrm{n}$ ) and the hop rate $h$ is roughly constant. (b) $E_{a d s}=E_{p}(\underline{x})$ and $E_{T S}=c$ so $E_{a c t}(i \rightarrow f)=c-E_{p}(i)$ produces weaker DA compared to (a). This follows as $\mathrm{E}_{\text {act }}$ is significantly higher and hop rates, $h$, are lower in regions of strongest binding, and this somewhat inhibits nucleation noting that the nucleation rate is proportional to the hop rate.

It is appropriate to also mention that DA can instead be kinetically-directed [134-135]. One scenario is: (c) constant $E_{a d s}$ and $E_{T S}=E_{d}+E_{p}^{\prime}(\underline{x})$ with constant $E_{d}$, so $E_{a c t}(i \rightarrow f)=E_{d}+\left[E_{p}^{\prime}(f)+E_{p}^{\prime}(i)\right] / 2$, where $E_{p}^{\prime}$ has the same form as $E_{p}$ but with distinct parameters $\Delta^{\prime}$, etc.

We expect that experimental observations for FF can be described by any of the choices (a-c), or other choices, with reasonable energetic parameters (cf. Ref. 135). However, limited DFT analysis [133,142] is more consistent with thermodynamically-directed DA. Thus, here we make the choice (a) specifically with $\Delta$ $=\delta$ and $\delta^{*}=0$ (cf. 133) adjusting $\mathrm{E}_{\mathrm{d}}$ and $\Delta$ to match the experimental behavior of FF versus $\theta$. In this case, it is reasonable to identify an effective total barrier for surface mass transport of $\mathrm{E}_{\mathrm{eff}} \approx \mathrm{E}_{\mathrm{d}}+\Delta$ (cf. Fig. 23). Below, we apply this model to elucidate the behavior for three specific metal on $\mathrm{G} 1 / \mathrm{Ru}(0001)$ systems. Model predictions for these systems for FF and $<\mathrm{s}>$ versus $\theta$ are compared with experiment in Fig. 21 (b,c,d).

\subsection{Application of modeling to specific systems}

In the modeling described below, values of key energies are primarily regarded as free parameters, and determined by fitting experimental observations. This approach has been particularly effective for simpler homoepitaxial growth systems, sometimes giving more reliable assessment of key energies than obtained from semi-empirical or DFT analysis [78]. Also for the systems of interest here, DFT analysis of energetics is limited but has recently become more prevalent (see Sec.6.3), and should in the future be more frequently utilized as input to modeling of directed assembly

$R u / G 1 / R u(0001)$. The adsorption energy for $\mathrm{Ru}$ on $\mathrm{G} 1 / \mathrm{Ru}(0001)$ of $-2.6 \mathrm{eV}$ [138] is similar to that on free-standing G1 of $-2.0 \mathrm{eV}$ [134]. Thus, one might expect that $\mathrm{E}_{\mathrm{d}}$ on $\mathrm{G} 1 / \mathrm{Ru}(0001)$ is similar to the value of $0.62 \mathrm{eV}$ on free-standing G1[133-134]. Indeed, we find that behavior for $\mathrm{Ru}$ on $\mathrm{G} 1 / \mathrm{Ru}(0001)$ is described by choosing $\left(\mathrm{E}_{\mathrm{d}}, \Delta\right)=(0.55,0.47)$ or $(0.58,0.39)$ or $(0.61,0.32)$, etc. with energies in $\mathrm{eV}$ [134-135]. If one further utilizes the DFT analysis in Ref. 133 indicating that $\Delta \approx 0.40 \mathrm{eV}$, this suggests that $\mathrm{E}_{\mathrm{d}}$ is just below $0.58 \mathrm{eV}$.

$P t / G 1 / R u(0001)$. The adsorption energy for $\mathrm{Pt}$ on $\mathrm{G} 1 / \mathrm{Ru}(0001)$ of $-2.8 \mathrm{eV}$ [138] is much stronger than that on free-standing G1 of $-1.6 \mathrm{eV}$ [139]. Thus, one expects that $\mathrm{E}_{\mathrm{d}}$ for $\mathrm{Pt}$ on $\mathrm{G} 1 / \mathrm{Ru}(0001)$ is much stronger than the value of $0.15 \mathrm{eV}$ [139] for free-standing G1. Indeed, the behavior for $\mathrm{Pt}$ on $\mathrm{G} 1 / \mathrm{Ru}(0001)$ is only described by much higher $\mathrm{E}_{\mathrm{d}}$ 
choosing $\left(\mathrm{E}_{\mathrm{d}}, \Delta\right)=(0.55,0.42)$ or $(0.58,0.35)$ or $(0.61,0.28)$, etc. with energies in $\mathrm{eV}$ [139]. These results imply a higher effective mobility for Pt than for $\mathrm{Ru}$ (due to the lower $\Delta$-values), consistent with a lower FF.

$R h / G 1 / R u(0001)$. The adsorption energy of $\mathrm{Rh}$ on $\mathrm{G} 1 / \mathrm{Ru}(0001)$ is similar to that for $\mathrm{Ru}$ [138]. However, the experimental behavior is described by choosing $\left(\mathrm{E}_{\mathrm{d}}, \Delta\right)=(0.55$, $0.36)$ or $(0.58,0.29)$ or $(0.61,0.22)$, etc. with energies in $\mathrm{eV}$, so $\Delta$ is lower than for $\mathrm{Ru}$. This implies a higher effective mobility for $\mathrm{Rh}$ than for $\mathrm{Ru}$ and $\mathrm{Pt}$ (consistent with the lower FF values for Rh).

KMC simulation of our model has provided insight into other aspects of behavior for $\mathrm{Ru}$ and $\mathrm{Pt}$ on $\mathrm{G} 1 / \mathrm{Ru}(0001)$ at $\sim 300 \mathrm{~K}$ where there is significant inter-cell transport [135-136]: (a) We find that $\mathrm{FF} \sim \theta^{1 / 3}$ consistent with irreversible NC formation. (b) NC size distributions have the form $\mathrm{P}_{\mathrm{s}} \propto \mathrm{f}(\mathrm{s} /<\mathrm{s}>)$ with "broad" width scaling like $<\mathrm{s}>$. (c) Height distributions are obtained from our point-island models by assigning size thresholds, $\mathrm{s}_{\mathrm{h} \rightarrow \mathrm{h}+1}$, for transitions from height $\mathrm{h}$ to $\mathrm{h}+1$ layer NC. To fit experiment at $\sim 300 \mathrm{~K}$, we can choose $\mathrm{s}_{\mathrm{h} \rightarrow \mathrm{h}+1}=7,25,60, \ldots(9,38,70, \ldots)$ for $\mathrm{Ru}(\mathrm{Pt})$ on $\mathrm{G} 1 / \mathrm{Ru}(0001)$. (d) Finally, the observed anti-clustering of NC distributions, which is most prominent for $\mathrm{FF} \approx 0.25-0.50$, is recovered quantitatively by our modeling.

$(R u+P t) / G 1 / R u(0001)$. Engstfeld et al. [139-140] sequentially deposited on $\mathrm{G} 1 / \mathrm{Ru}(0001)$ first less mobile $\mathrm{Ru}$ and then more mobile $\mathrm{Pt}(\mathrm{Pt} @ \mathrm{Ru}$ ) producing primarily 3D Ru core-Pt shell NCs. First depositing more mobile Pt then less mobile Ru produces many pure Ru NCs in addition to 3D Pt core-Ru shell NCs. Atomistic modeling with the parameters described above is able to recover FF both after the first and second stages of deposition. This modeling elucidates the experimentally-observed NC height distributions as shown in Fig.24 for Ru@Pt, and produces the size distributions shown in Fig. 25 [140]. Experimentally observed and simulated NC distributions for the second case are shown in Fig. 26 [139].

\subsection{DFT analysis of energetics for metals on metal-supported graphene}

DFT analysis of the energetics for metals on metal-supported graphene would provide key input for atomistic-level modeling of the type described above. However, the large moiré cell makes any such calculation computationally demanding, and the complex potential energy landscape for an adsorbed metal atom would require extensive analysis for comprehensive characterization. However, a more targeted or simplified analysis is useful to extract at least the key features of the energetics, and is being performed with increasing frequency.

The first DFT analysis aimed to explain the binding of $\operatorname{Ir}$ to G1/Ir(111), in particular to elucidate the stronger binding in hcp-type and fcc-type regions of this substrate relative to on-top regions [49]. The argument was that in these regions 3 out of $6 \mathrm{C}$ atoms in a carbon ring sit on top of substrate Ir atoms, forming covalent bonds to the substrate through hybridization of $\mathrm{C} 2 p_{z}$ orbitals and Ir $d$ orbitals. This disturbed the graphene $\pi$-bonds, thus activating the remaining $\mathrm{C}$-atoms for bonding to Ir adatom clusters.

In another early study of relevance for the above analysis of $\mathrm{Ru}$ on $\mathrm{G} 1 / \mathrm{Ru}(0001)$, Sutter et al. [133] explored the binding of $\mathrm{Ru}$ atoms and clusters (trimers) in different regions of the moiré cell. The fcc region was shown to be the preferred binding location for $\mathrm{Ru}$, the adsorption here being more stable by $\Delta \approx 0.4 \mathrm{eV}$ than in the hcp region. This result is consistent with experimental observations for nucleation of Ru NCs. They also 
showed that the $\mathrm{Ru}$ atom occupies a position in the center of the $\mathrm{C}$ ring. $\mathrm{Ru}$ trimers were shown to bind flat on the graphene sheet above $\mathrm{C}$ atoms, their strong interaction lifting the sheet.

Additional DFT analysis has also been performed for adsorption energies and potential energy surfaces (PES) of $\mathrm{Rh}$ and $\mathrm{Au}$ adatoms on $\mathrm{G} 1 / \mathrm{Ru}(0001)$. Results using the full $(12 \times 12) \mathrm{G} /(11 \times 11) \mathrm{Ru}$ moiré cell were used to determine the global diffusion barrier across the surface $(0.25 \mathrm{eV}$ for $\mathrm{Rh}$ and $0.81 \mathrm{eV}$ for $\mathrm{Au})$. These values account for the local barrier and long range modulation of the PES as described in Sec.6.1. These long range diffusion barriers were then "reconstructed" using energetics obtained from "small surface models" representing different local regions of the moiré superstructure, a process which somewhat consistently predicted $0.53 \mathrm{eV}$ for $\mathrm{Rh}$ and $0.71 \mathrm{eV}$ for $\mathrm{Au}$ [145]. With this proof-of-principle, more extensive analogous analysis was performed for eighteen 4d (Y-Ag) and 5d (La-Au) transition metal adatoms on G1/Ru(0001) [146]. Correlation between global diffusion barriers and adsorption energies were also explored with an aim of elucidating nucleation and growth of NCs on these surfaces. We would note however that analysis of nucleation processes is subtle for these directed assembly processes as indicated by our modeling in Sec.6.1 and 6.2. Thus, simple correlation with, e.g., a global diffusion barrier, might not apply.

Finally, with regard to other more comprehensive studies, Wang and Bocquet [138] compared adsorption energies form various TM metals ( $\mathrm{Ru}, \mathrm{Rh}, \mathrm{Pt}, \mathrm{Pd}, \mathrm{Au}, \mathrm{Ni}, \mathrm{Co}, \mathrm{Fe}$, $\mathrm{Cu}, \mathrm{Ag}$ ) on $\mathrm{G} 1 / \mathrm{Ru}(0001)$ with the TM-TM interaction in small TM clusters (dimers and trimers). The relative magnitude of these energies was used to assess the likely growth mode (cf. Sec.7.1). We also mention another recent and extensive DFT analysis of adsorption energies of $\mathrm{Pt}, \mathrm{Ru}, \mathrm{Ir}, \mathrm{Ti}, \mathrm{Pd}, \mathrm{Au}, \mathrm{Ag}$, and $\mathrm{Cu}$ adatoms on $\mathrm{G} 1 / \mathrm{Ru}(0001)$, compared with DFT results on freestanding G1 [147]. We remark that there are some inconsistencies with previously published results as well as with our own analysis [148].

\section{General trends in growth morphology and stability}

\subsection{Growth morphology}

From the examples discussed above, we can see that different metals on graphene exhibit distinct growth morphologies. The nanocluster (NC) growth morphology is also dependent on the substrate supporting the graphene. The different growth morphologies (i.e., 2D vs 3D) can be attributed to the different interactions between the metals and graphene. A rigorous analysis of this dependence has been formulated in terms of relevant surface and adhesion energies [149]. However, for future experimental design of metal nanostructures on graphene, it is useful if some more simply implemented general rules or guidelines that relate the fundamental interactions to the growth morphology can be established. From the standpoint of thermodynamics, we proposed that the ratio between adsorption energy of the metal adatom on graphene and the bulk binding energy of the metal, $E_{a} / E_{b}$, should play an important role in governing the dimensionality of growth (i.e., 2D or 3D) [57-59]. For a large $E_{a} / E_{b}$ value, metal adatoms are more stable if they can preserve contact with graphene, and growth will likely be $2 \mathrm{D}$, at least at sub-monolayer coverage where the metal-graphene contact is most important. On the other hand, 3D growth will take place if the ratio $E_{a} / E_{b}$ is small since in this case it is more energetically favorable for the metal adatoms to be in contact with other metal atoms rather than the graphene substrate. Note that the above analysis implicitly assumes 
that nanocluster shapes are equilibrated. If not then the Ehrlich-Schwoebel step edge barrier also plays a role in assessing $2 \mathrm{D}$ versus 3D morphology as it impacts interlayer transport. The $E_{a} / E_{b}$ values are still not available from experiment for most metal on graphene because the adsorption energy $E_{a}$ is not easily measured by experiment. On the other hand, there have been many first-principles DFT calculations of $E_{a}$ for various metals on free-standing graphene. Therefore, the $E_{a} / E_{b}$ values for metals on free-standing graphene can be used as guidelines to access the growth morphology of metal on graphene provided the interaction between graphene and substrate is weak such as in the case of $\mathrm{SiC}$ - and $\mathrm{SiO}_{2}$-supported graphene.

As shown in Table 1, most of the metals (except alkali metals and most of the REMs) on free-standing graphene have $E_{a} / E_{b}<0.5$. In particular, the $E_{a} / E_{b}$ ratios for $\mathrm{Cr}$, $\mathrm{Mn}, \mathrm{Pb}, \mathrm{Cu}, \mathrm{Ag}$, and $\mathrm{Au}$ on graphene are extremely small. According to the $E_{a} / E_{b}$ rule, metal on free-standing graphene (or on graphene which is weakly bonded to the supported substrate such as $\mathrm{SiC}$-supported graphene) with small $E_{a} / E_{b}$ ratio should favor a 3D growth mode. This prediction agrees well with experimental results of metal on $\mathrm{SiC}$ (0001)-supported graphene discussed above where 3D growth mode has been observed for most of the metals studied. According to this rule, alkali metals especially $\mathrm{K}$ and $\mathrm{Cs}$ and $\mathrm{RE}$ metals $\mathrm{Nd}$ and $\mathrm{Sm}$ on graphene should exhibit a 2D growth. Indeed, 2D growth of $\mathrm{K}$ and $\mathrm{Cs}$ on graphene supported by $\mathrm{SiC}$ has been observed by experiments.

3D metal growth on graphene is a major limitation for many graphene applications (i.e., to grow reproducible metal contacts of low resistance or to fabricate spin filters) that require the metal to follow layer-by-layer growth. On the other hand, the $E_{a} / E_{b}$ rule also points to an approach to control the growth morphology from $3 \mathrm{D}$ to $2 \mathrm{D}$ by manipulating the adsorption energy of the metals by engineering the substrate that supports the graphene. In fact, such an approach has been used in several experiments but the connection to $E_{a} / E_{b}$ has not yet been established. For example, the propensity of Ir, Pt, W and $\operatorname{Re}$ to grow in a $2 \mathrm{D}$ mode on $\operatorname{Ir}(111)$-supported graphene has been seen in the examples discussed in Section 5.1 (see Fig. 22). 2D growth on graphene has been demonstrated to be feasible with Co films deposited on graphene grown on $\operatorname{Ir}(111)$ [8] and for $\mathrm{Au}$ on $\mathrm{Ru}(0001)$ supported graphene [90]. Although the 2D growth of $\mathrm{Co} / \mathrm{G} 1 / \operatorname{Ir}(111)$ was attributed to an effective high flux rate, it would be interesting to investigate the adsorption energy of $\mathrm{Co}$ on $\mathrm{G} 1 / \operatorname{Ir}(111)$ and $\mathrm{Au}$ on $\mathrm{G} 1 / \mathrm{Ru}(0001)$.

According to the $E_{a} / E_{b}$ rule, co-deposition of metals on graphene and intercalation (which will be discussed in Sec. 10) would also provide other knobs to tune the $E_{a} / E_{b}$ values and thus the growth morphology of metal on graphene. N'Diaye et al. [106] pre-deposited a small amount of $\operatorname{Ir}$ on $\mathrm{G} 1 / \operatorname{Ir}(111)$ to control and pin the location of islands of subsequently deposited $\mathrm{Au}$ and $\mathrm{Fe}$ which would otherwise not form ordered NC arrays. Another example was discussed at the end of Sec. 6.2. The growth morphology of $\mathrm{Pt}$ and $\mathrm{Ru}$ on $\mathrm{G} 1 / \mathrm{Ru}(0001)$ is strongly dependent on the sequence of $\mathrm{Pt}$ and $\mathrm{Ru}$ depositions [139-140].

In addition to the 2D vs 3D growth morphology, the density and shape of islands on graphene are dependent on the surface diffusion barrier $E_{d}$ and the edge diffusion barrier(s) $\mathrm{E}_{\mathrm{s}}$ which controls intralayer diffusion. In standard nucleation theory [78,122], the $\mathrm{NC}$ density $\mathrm{N}$ increases as the diffusion coefficient decreases: low $\mathrm{N}$ implies fast diffusion while high $\mathrm{N}$ indicates slow diffusion, if flux, coverage, and critical size are the same. Therefore, the NC density observed by STM experiment can be correlated with the 
diffusion barriers of adatoms on graphene. We should mention that this analysis refers to "homogeneous" nucleation on perfect terraces. In practice, experimental analysis of island densities, and more generally of island size distributions, is performed by acquiring statistics from perfect regions of the graphene sheet far separated from any defects or edges. Then, the standard theory applies and produces valuable insights. The shape of the $\mathrm{NCs}$ is also related to the edge diffusion barrier $\mathrm{E}_{\mathrm{s}}$ of the metal adatoms. In general, for (surface) diffusion-mediated growth of NCs, one anticipates a Mullins-Sekerka or Diffusion-Limited Aggregation (DLA) type shape instability leading to fractal or dendritic shapes. However, this shape instability is often quenched by shape relaxation due to edge diffusion. Thus, the fractal shapes are classically attributed to limited edge diffusion $[78,122]$.

The diffusion barrier $\mathrm{E}_{\mathrm{d}}$ of many metal adatoms on free-standing graphene has been studied by first-principles calculations as shown in Table 1. In general, correlation between experimentally observed island density and calculated adatom diffusion barrier can be observed. For example, for $\mathrm{Cr}, \mathrm{Mn}, \mathrm{Cu}, \mathrm{Pb}$, and $\mathrm{Au}$ on graphene not only their $E_{a} / E_{b}$ ratios but also their diffusion barriers are extremely small resulting in relatively large NC size and small NC densities for these metals on graphene even below room temperature. On the other hand, a higher NC density and smaller NC size would be expected for other metals on graphene, especially for $\mathrm{Fe}, \mathrm{Co}, \mathrm{Ni}, \mathrm{Pt}$, and $\mathrm{Gd}$ because these adatoms have relatively large diffusion barrier. The predictions from the first-principles calculations agree well with experiments. However, there are still some exceptions. We note that both $\mathrm{Dy}$ and $E u$ have very similar $E_{a} / E_{b}$ ratio and diffusion barriers $E_{d}$, yet their growth morphologies are quite different. This result suggests that other factors (e.g., lattice distortion and elastic interactions) affect the growth morphology. Moreover, there are almost no detailed calculations extracting the edge diffusion barrier(s) $\mathrm{E}_{\mathrm{e}}$ and no attempts to relate $\mathrm{E}_{\mathrm{e}}$ to the observed growth morphology.

\subsection{Thermal stability of metal NC}

The thermal stability of the metal NCs grown on graphene is an important property that affects many applications. For example, due to its high surface area and low manufacturing cost, graphene has been used as substrate for surface-supported catalysis. Graphene-supported nanocatalysis with Pt [150-153], Pd [154], Au [155-157], and metal alloys [158-163] has been reported. The thermal stability of transition and noble metal nanostructures on graphene is crucial for the performance of the catalysis applications. Graphene also shows considerable promise as lithium reservoirs for potential application in electrochemical storage devices, such as rechargeable Li batteries. Experiments show that graphene nanosheets and oxidized graphene nanoribbons can adsorb higher amounts of $\mathrm{Li}$ than conventional graphite [164-166]. In lithium-ion batteries, the stability of cathode or anode materials is a key factor for the charging efficiency.

The classic theory of coarsening via Ostwald Ripening (OR) identifies the effective overall barrier for OR as the sum of the diffusion barrier, the formation energy (to create a terrace adatom from the NC), and any additional attachment barrier. For systems of interest, often the diffusion barrier is small, and we do not expect an additional attachment barrier. Thus, coarsening is mainly controlled by the formation energy which corresponds to the difference between the bulk binding energy and adsorption energy of the metals on graphene. Consequently, this energy difference, $E_{b}-E_{a}$ has been proposed to 
be a convenient parameter to measure the thermal stability of metal nanostructures on graphene [56-59]. For a metal with small $E_{b}-E_{a}$, coarsening of the metal islands will take place easily because atoms can easily detach from small NCs and diffuse over graphene to join other bigger NCs. On the other hand, larger $E_{b}-E_{a}$ will require higher temperature for NC coarsening and the metal NCs will be more stable.

According to this criterion and the first-principles calculation results shown in Table 1, coarsening of alkali metal and some rare earth metal ( $\mathrm{Sm}$ and $\mathrm{Eu}$ ) NCs should take place at relatively low temperatures. On the other hand, transition metals should exhibit higher thermal stability against coarsening. The stability against coarsening for the group III and IV metal NCs and other rare earth NCs on graphene is lower than that of the transition metals but should be much higher than that of the alkali metals. The $E_{b}-E_{a}$ criterion suggests that graphene supported transition metals (including $\mathrm{Pd}$ and $\mathrm{Pt}$ ) should be the most promising systems for supported catalysis because the Pd and Pt NCs on graphene should exhibit high thermal stability. For noble metals $\mathrm{Cu}, \mathrm{Ag}$, and $\mathrm{Au}$ on free-standing graphene, $\left(E_{b}-E_{a}\right)$ values are large, but the adsorption energy is very small and diffusion barriers nearly zero. Therefore, NCs composed of these noble metals on free-standing graphene may not be very robust for catalysis applications. However, adsorption of noble metals on metal-supported graphene can change their adsorption energy and diffusion barrier (particularly noting the periodic modulation induced by rumpled graphene) and thus the thermal stability.

Using STM, the thermal stability of several metals (Fe, Gd, Dy, and Eu) on graphene has been investigated. The metals were initially deposited at room temperature on graphene prepared by thermal annealing of $\mathrm{SiC}$ [90]. After characterization of the growth, the metal NCs were annealed to a higher temperature. The NC morphologies before and after annealing measured by STM are compared as shown in Fig. 27. The experimental results indicate that $\mathrm{Fe}, \mathrm{Gd}$, and Dy are very stable against coarsening, while coarsening was clearly observed for Eu after annealing to $365 \mathrm{~K}$, consistent with the predictions from the theoretical calculations since the $E_{b}-E_{a}$ value of Eu on graphene is small (see Table 1 and Fig. 27). In another experiment, it was also shown that $\mathrm{Pd}$ nanoparticles on graphene are very stable against aggregation above $800^{\circ} \mathrm{C}$ [167] which also is consistent with results for $E_{b}-E_{a}$ from DFT calculations. Co NCs on graphene are found to be stable up to $650 \mathrm{C}^{\circ}$ before intercalation taking place at higher temperature [72]. Recently, it has been shown that $\mathrm{Ni}$ and $\mathrm{Mn}$ nanoparticles have relatively higher thermal stability, up to 800 and $600 \mathrm{C}^{\circ}$ respectively [69]. All these experimental studies indicate that transition metals on graphene require a higher temperature to activate aggregation or desorption, which is considered as a necessary feature of good surface catalyst and hydrogen storage materials. The prediction of thermal stability of Pt and Ir on graphene from $E_{b}-E_{a}$ is also supported by experiment. Fig. 28 shows the annealing sequence of Pt cluster arrays on graphene on $\operatorname{Ir}(111)$ up to $650 \mathrm{~K}$. The Pt cluster superlattice remains intact up to $400 \mathrm{~K}$.

\section{Electronic and Magnetic properties}

Another interesting issue for metals on graphene is how adsorption of the metal affects the electronic and magnetic properties of graphene, as well as of the metal. The electronic and magnetic properties of various metals on free-standing graphene have been 
well studied by first-principles calculations as shown in Table 1. In Fig. 29, we also show the electronic density-of-states (DOS) of some typical metals adsorbed on graphene. Most alkali metals on graphene donate their outer shell electron to graphene and raise the Fermi level of graphene above the Dirac point while keeping the intrinsic band structure of graphene intact. It can be seen from Fig. 29 that the Dirac point and the DOS of graphene below the Dirac point are well preserved upon $\mathrm{Na}$ and $\mathrm{K}$ adsorption, but the Fermi level shifts up $\sim 0.85 \mathrm{eV}$ away from the Dirac point due to electron transfer from the alkali-metal adatoms to the graphene. In the case of Al and In adsorption, the Dirac point and the DOS of graphene below the Dirac point are also well preserved, although there is a small distortion due to some degree of covalent bonding. The electron transfer from the $\mathrm{p}$ orbitals of $\mathrm{Al}$ and In adatoms to graphene shifts the Fermi level up $0.90 \mathrm{eV}$ away from the Dirac point. However, the transferred electrons occupy the spin-up and spin-down subbands above the Dirac point symmetrically and induce no magnetic moments. Transition metals on graphene exhibit strong covalent interactions between the metal adatom and carbon atoms of graphene and significantly modify the electronic structure of graphene. An important common character in the DOS shown in Fig. 29 for 3d-transition-metal adatoms on graphene is the strong hybridization between $\mathrm{d}$ states of the adatoms and the $\mathrm{p}_{\mathrm{z}}$ state of graphene. The $\mathrm{d}$ valence states of the 3d-transition-metal adatoms are well below the Fermi level and interact strongly with graphene, and destroy the Dirac point in the electronic structure of pure graphene. The occupation of the spin-up and spin-down states is very uneven for Fe and $\mathrm{V}$ adatoms on graphene, suggesting large magnetic moments. For $\mathrm{Co}$ and $\mathrm{Ni}$ on graphene, the occupation of the spin-polarized d states is almost symmetric, resulting in a much smaller magnetic moment. In comparison with alkali- and group-III metal adatom adsorption, the interaction between the $3 \mathrm{~d}$-transition-metal adatoms and graphene is much stronger.

For transition metal adatoms and nanoclusters that carry magnetic moments, binding to graphene presents a chemical route to localized magnetic moments in graphene and may facilitate development of future graphene-based spintronic and microelectronic devices. Recently, scanning tunneling microscopy, x-ray adsorption spectroscopy, and $\mathrm{x}$-ray magnetic circular dichroism (XMCD) techniques have been used to investigate the adsorption behavior of TM adatoms on graphene [79-85]. The XMCD proves Fe and Co adatoms to be paramagnetic with high spin configuration, and $\mathrm{Ni}$ adatoms exhibit a nonmagnetic ground state [83] as one can see from Fig. 30. It is worth noting that the spin configuration is also substrate-dependent $[80,168]$. Cobalt atoms on $\mathrm{G} 1 / \mathrm{Ru}(0001)$ feature a large magnetic moment which indicates high spin state. By contrast, the magnetic moment is strongly reduced due to hybridization with $\mathrm{G} 1 / \operatorname{Ir}(111)$ [80-81]. Another experiment [168] also reports similar observations that the ground spin state is substrate dependent for Fe and Co adatoms on graphene supported by highly-oriented pyrolitic graphite and few layer graphene, respectively [83].

In addition to isolated metal adatoms, the magnetic properties of metal clusters on graphene [169-178] have also attracted much more attention recently. Metal clusters on graphene introduce magnetic moment in graphene [175-176] which can be used in ferromagnetic spin injectors. As discussed in Sec. 2.2, formation of $3 d$ transition metal clusters on graphene has been observed in several experiments [79-85]. Theoretical calculations suggested that clusters of Mn [69], Fe [70,74], Co [71-73], Ni [70,73] on graphene are energetically favorable [175-176]. However, the magnetic moments of most of the transition metal clusters on graphene are reduced from the values in the corresponding free-standing clusters due to charge redistribution caused by interaction 
with graphene substrate [175-176]. Mn clusters on graphene are exceptional. Using DFT calculations it was shown that a $\mathrm{Mn}_{7}$ cluster on graphene exhibits a magnetic moment enhancement. The cluster on graphene has a moment of $6.3 \mu_{\mathrm{B}}$ which is larger than $5.0 \mu_{\mathrm{B}}$ in an isolated $\mathrm{Mn}_{7}$ cluster by $26 \%$ [176]. The spin-polarized electron density distribution in $\mathrm{Mn}_{7}$ on graphene has also been examined as one can see in Fig. 31. The figure shows that the magnetic moment is mainly from the $\mathrm{Mn}_{7}$ cluster (Fig. 31(b)). The total net magnetic moment of the $\mathrm{Mn}_{7}$ cluster is about $6.0 \mu_{\mathrm{B}}$. In addition, $\mathrm{Mn}_{7}$ adsorption induces a magnetic moment of $0.3 \mu_{\mathrm{B}}$ in the graphene layer. Indeed, Fig. 31 shows that the carbon atoms beneath the Mn cluster exhibit noticeable magnetic moments. There are spin-up states next to the Mn atoms and spin-down charge densities in the next shell, based on the 2D contour plot shown in Fig. 31(c). The integration of spin-polarized charge density on all carbon atoms is positive. Therefore, there is a net magnetic moment from graphene which contributes to the enhancement of the magnetic moment in the $\mathrm{Mn}_{7} /$ graphene system. The behavior of the magnetic moment of Mn clusters on graphene [176] is in contrast to other transition metal clusters on graphene.

\section{Metals on graphite: Comparison to graphene}

\subsection{General comments}

Graphite and graphene are similar yet different. Whereas graphite is a semi-metal, having a small conduction band, graphene is a zero-bandgap semiconductor [179]. Graphite has only a third of the charge density of graphene [180], and its surface energy is $17 \%$ higher $\left(54.8 \mathrm{~mJ} / \mathrm{m}^{2}\right.$ vs. $46.7 \mathrm{~mJ} / \mathrm{m}^{2}$, respectively) [181]. From a chemical bonding point of view, van der Waals forces exist between carbon sheets in graphite but are obviously lacking for isolated graphene sheets. For all of these reasons and more, one expects adsorption of metals on graphite and graphene to be at least somewhat different.

In terms of energetic parameters, such as the adsorption energy and the diffusion barrier for metals on these two carbonaceous substrates, there are only a few metals for which comparisons can be made. All of the comparisons are based on DFT, and most are for transition metals $(\mathrm{Cr}, \mathrm{Pt}, \mathrm{Cu}, \mathrm{Ag}, \mathrm{Au})$. These values have been reviewed and compiled recently [182]. Comparison between the best available estimates of adsorption energies is also reported here in Table 2. For this limited range of metals, the adsorption energy of a metal atom on graphene is consistently and significantly lower than that on graphite, while the diffusion barrier is lower than or comparable to that on graphite.

Notably, differences between metal adsorption on graphene and graphite are, in a sense, being approached by the experimental comparisons of metal bonding to n-layer graphene that were reviewed in Section 3. Metal bonding depends strongly on the number of carbon layers, at least in the few-layer regime. However, there appear to be no experimental comparisons between true graphite and graphene with regard to metal adsorption and nucleation. This is an area that is still in the very early stages of exploration.

\subsection{Case study: Cu deposition on graphite}

Despite extensive studies of the formation of metal NCs by deposition on graphite, a detailed and rigorous connection of the clusters characteristics to the underlying nucleation process has rarely been provided [71,183-185]. Here, we just describe one recent detailed analysis for $\mathrm{Cu}$ deposition on HOPG where the default deposition process utilized an e-beam heater. Fig. 32 presents STM images for deposition at room temperature revealing 
the formation of 3D Cu NCs on broad terraces of HOPG, together with a more extensive decoration of steps on the substrate. Quantitative analysis of the mean island or NC density, and the island size distribution from such data is shown in Fig.33. Separate DFT analysis indicated a $\mathrm{Cu}$ diffusion barrier of $\mathrm{E}_{\mathrm{d}}=0.020 \mathrm{eV}$ and an adsorption energy of $\mathrm{E}_{\mathrm{a}}=$ $0.589 \mathrm{eV}$ for this system. However this $\mathrm{E}_{\mathrm{d}}$ would imply an $\mathrm{NC}$ density at least an order of magnitude lower than the experimental value assuming homogeneous nucleation corresponding to irreversible island formation with a critical size $i=1$ (and even lower value allowing reversibility in this process). Considering various modifications to the simplest nucleation picture (adding an extra barrier for aggregation and nucleation, suppression of island growth above a threshold size, initiation NC nucleation via a place-exchange event often labeled as $\mathrm{i}=0$ ) can improve some aspects of the model agreement with experiment, but none can match all of the mean island density, the linear increase with coverage, and the monotonically decreasing island size distribution shown in Fig.33.

The resolution of the difficulty in model NC formation came from the realization that a small fraction, $\mathrm{p} \approx 5 \times 10^{-4}$, of ions in the deposition source were responsible for ion-mediated heterogeneous nucleation. Specifically, each such ion induces localized damage of the HOPG surface which acts as a nucleation center for $\mathrm{Cu} \mathrm{NC}$ formation. This picture was confirmed by performing additional experiments without crucible bias and filament current to eliminate the presence of ions, showing a lack of NC formation on terraces. Simulation and analytic treatment of the model of ion-mediated nucleation described all aspects of the experimental observations as indicated in Fig.33. The linear increase in NC density with coverage is immediately clear, as is the generation of a random distribution of NCs on the surface. Simulations confirm the monotonically decreasing nature of the NC size distribution. An additional analysis considered the inhibition of nucleation as a result of the diminution of the portion of the surface not covered by the footprint of the 3D NCs. It was shown that a suitably refined Johnson-Mehl-Avrami-Komogorov formulation capturing this inhibition could recover experimental observations. See also Fig. 33.

\section{Intercalation for supported graphene systems and for graphite}

Surface-tailored intercalation of metals in layered compounds offers a versatile way to modify the surface properties of these materials. It has the potential to form two-dimensional metal layers that are chemically and mechanically protected, spatially localized, and functionally tailored. The combination of intercalated surface islands plus the modified overlayer could be useful in magnetic storage devices, micro- or nano-mechanical systems, and plasmonics.

There are striking examples showing that intercalation can change the surface properties of graphene. Inhomogeneous graphene substrates with mixed areas of different intercalation have been prepared for graphene grown on $\mathrm{SiC}(0001)$ [186]. With Ge used as intercalant, it was possible to generate three types of regions: pristine, n-doped (1-layer $\mathrm{Ge}$ ), and p-doped (2-layer $\mathrm{Ge}$ ) areas that have potential use as in-plane transistors [63,64,186-187]. Superconductivity in Ca-intercalated multilayer epitaxial graphene films on $\mathrm{SiC}$ has also been reported [188]. Transition metal [71-72] intercalation (e.g., Co on G1/Ir(111)) was also shown to have dramatic effects on the magnetic properties of graphene [189]. In a following section, we give examples showing that nucleation and growth of metals on graphene is also strongly affected by intercalation. 
Another promising property is the ability of the overlying carbon layer to shield and pacify any underlying metal [190]. For instance, surfaces of $\mathrm{Cu}$ and $\mathrm{Ni}$ coated by monolayer graphene are protected from oxidation, even under quite challenging conditions (elevated temperature in air [191] or aqueous electrochemical environments [192-193].) Several reports also indicate that intercalated metals are oxidation-resistant [53,194-195]. Remarkably, Co oxide nanoparticles on top of graphene/SiC(0001) actually undergo de-oxidation, and the metallic Co intercalates, when heated in vacuum. Subsequently, the intercalated Co is not susceptible to re-oxidation [53].

For layered carbon, there are essentially two classes of intercalation systems. One class is based on bulk compounds of graphite, which are well-known and diverse; here, the intercalant occupies the galleries between carbon sheets [196-199]. The other is based on supported graphene [16], where the intercalant is at or near the graphene-support interface, often modifying the electronic properties of the graphene blanket while leaving its structural integrity intact.

Interesting and active areas of inquiry are described in more detail below, for both classes of intercalation systems they include the way in which intercalation changes surface properties as well as and the driving force, mechanism, and kinetics of intercalation. The spatial density and distribution of the intercalant is also of interest.

\subsection{Effect of intercalation on surface metal growth}

While experiments on exfoliated graphene of various thicknesses from single to multilayers have shown that the island density can depend dramatically on the carbon layer thickness for $\mathrm{Cs}$ [37] and for $\mathrm{Au}[16,17]$, recent experiments also show that metal intercalation [35,186,200-202] provides a way to effectively modify the properties of graphene and tune the adatom bonding. It has been shown that adsorption of metal atoms can be manipulated by the insertion of metallic layers between graphene and the supporting substrate [202]. By preparing a graphene sample on $\operatorname{Ir}(111)$ such that intercalated and non-intercalated regions coexist (i.e. partial intercalation), Schumacher et al. [201-202] showed that nucleation of additional metal (Eu or Cs) is concentrated on top of the pristine areas, while the island density on the intercalated regions is essentially zero.

A key question is mass transport, i.e., how atoms deposited uniformly on graphene populate different areas depending on the local intercalation of the area. In a study by Michely et al., strong selectivity for adsorption of $\mathrm{Eu}$ and $\mathrm{Cs}$ on pristine G1/Ir(111), compared to regions intercalated with $\mathrm{Eu}$ or $\mathrm{Cs}$, was attributed to the adsorption energy difference between the pristine and modified areas [202]. Nevertheless, it is also necessary to address the question of mass transport, since the deposition process initially distributes adatoms uniformly on intercalated and pristine areas. The metal adatoms must be able to transfer very efficiently to the areas where islands nucleate.

Very recently, we proposed a mechanism that leads to selective adatom mass transport on partially intercalated graphene through the electric field induced by inhomogeneous electron doping [203]. Using first-principles calculations, we showed that Eu and Cs intercalation can induce highly n-doped graphene patches by charge transfer to graphene. This redistribution of the electrons makes the electrostatic potential lower in the intercalated graphene areas and thus induces a strong electric field across the boundary between the intercalated and non-intercalated domains. This field provides significant driving force for biased diffusion of the positively charged metal adatoms toward the 
non-intercalated domains and can account for the unusual nucleation observed exclusively on these areas.

\subsection{Driving force and mechanism for intercalation and the intercalant distribution}

In supported graphene, the driving force for intercalation involves interaction with the support, as well as with the upper carbon layer. Reactive supports have included single crystals of all of the late transition metals, such as $\mathrm{Cu}(111), \mathrm{Ni}(111), \mathrm{Ru}(0001), \operatorname{Ir}(111)$; and of some carbides, notably $\mathrm{SiC}(001)$. Elemental intercalants in this class include hydrogen, oxygen, fluorine, silicon, germanium, indium, and a variety of metals spanning the alkali metals, alkaline earths, transition metals, and rare earths.

In bulk graphite, intercalation is driven by charge transfer to or from the amphoteric carbon sheets, which is balanced against the strain introduced by the intercalant and the disruption of the van der Waals interaction between sheets. The presence of strain is evident in the fact that a quantity called the staging index (the number of carbon layers separating layers of intercalant along the c-axis) can take values as high as 10. Effectively, this is because the elastic interaction between adjacent carbon sheets is repulsive $[197,198]$. Intercalation compounds of graphite are generally stabilized by small ionic radius and low ionization potential, reflecting the competing influences of strain and charge transfer.

The mechanism for intercalation involves complex diffusion processes along and between layers, and the stable states depend sensitively upon van der Waals interactions. The microscopic mechanisms and dynamics of these processes are not well understood. Several mechanisms for spontaneous surface intercalation under supported graphene have been considered in the literature, some justified more fully than others: (1) Diffusion from edges of graphene flakes toward the interior [204-207]; (2) Penetration at nanoscale features such as domain boundaries or nanoscale cracks formed by large compressive forces (wrinkles) [186,200,204,208]; (3) Intercalation though localized or point-like defects [209,210]; (4) Place-exchange between an adatom and a $\mathrm{C}$ atom in the atomically-perfect surface, followed by self-healing of the $\mathrm{C}$ sheet at high temperatures [206,209-213]; and (5) Formation of an intermediate carbide [214].

Inspection of the literature cited above indicates that the mechanism for metal intercalation depends strongly on the chemical nature of the metals and the structure of the carbon surface. For instance, Jin et al. [205] reported that $\mathrm{Ni}$ and $\mathrm{Pb}$ follow much different intercalation pathways (place exchange and step entry, respectively) for $\mathrm{G} 1 / \mathrm{Ru}(001)$ and attributed this to the difference in interaction strength of each metal with the carbon layer. Lahiri et al. reported formation of an intermediate carbide during intercalation of $\mathrm{Ni}$ but not $\mathrm{Cu}$, for graphene supported on $\mathrm{Ni}(111)$ [214,215]. Huang et al. [211] found that the rare earth Ce was unusual, in that it intercalated at $300 \mathrm{~K}$, in contrast to 6 other elements studied, Pt, Pd, Ni, Co, $\mathrm{Au}$, and $\mathrm{In}$, which all required heating to $500-800 \mathrm{~K}$, on $\mathrm{G} 1 / \mathrm{Ru}(001)$. It should be noted that by contrast, Schumacher et al. found that the rare earth Eu intercalates under G1/Ir(111) only at elevated temperatures [202].

There are at least two recent examples that illustrate the importance and versatility of the atomic arrangement of the intercalant. First is the case of $\mathrm{Ge}$ on graphene/SiC $(001)$, mentioned earlier, where buried $\mathrm{Ge}$ can be one- or two-layers thick, depending on annealing conditions, resulting in the overlying carbon sheet being $\mathrm{n}$ - or p-doped, respectively $[63,64,186,187]$. Second, Petrov et al. [200] have shown that Cs intercalates 
beneath $\mathrm{G} 1 / \operatorname{Ir}(111)$, in three phases with different densities, depending on experimental conditions. In the same vein, for bulk graphite it is well known that intercalation compounds can exhibit complex phase equilibria in which the density and structure of intercalants vary significantly [196-198].

\section{Summary}

In this article, we have reviewed recent experimental and computational studies of the interactions, growth morphologies, and stability of various metals on different types of graphene, i.e. graphene grown on $\mathrm{Si}$-terminated $\mathrm{SiC}$, graphene on $\mathrm{SiO}_{2}$, and graphene grown on metals. We should also note limited studies of metal growth on graphene supported on other substrates, e.g., Co deposited on $\mathrm{G} 1 / \mathrm{Zn}(0001)$ at room temperature and annealed to $350 \mathrm{C}^{\circ}$ [216].

For metals on graphene on the $\mathrm{SiC}$ substrate, experimental studies using physical vapor deposition and STM show that $\mathrm{Gd}$, $\mathrm{Dy}, \mathrm{Fe}, \mathrm{Eu}$, and $\mathrm{Pb}$ have very different growth morphologies and thermal stability although they all follow a 3D growth mode. $\mathrm{Pb}$ can form large crystalline NCs on graphene with multiple 10 layers heights even at very low temperature. Eu on graphene also forms large $\mathrm{NCs}$ and annealing at relatively low temperatures (below 400K) transforms the large NCs into an almost continuous film with thickness equal to the deposited amount, $\sim 3$ layers, covering the graphene fully. On the other hand, Gd, Dy, and Fe on graphene tend to have higher NC densities, indicating higher diffusion barriers generally associated with stronger interaction with graphene. In particular, Gd forms fractal-like 3-D NCs over a wide range of coverage and temperature. Most interestingly, the growth of Fe on graphene deviates from the prediction of classical nucleation theory. The density of Fe NCs does not reach saturation but grows almost linearly with coverage, and the NCs are essentially unchanged after annealing. This indicates the presence of repulsive interactions between the Fe adatoms which suppress monomer aggregation to preexisting NCs.

For metal nanostructures on few-layer graphene (FLG) supported on $\mathrm{SiO}_{2} / \mathrm{Si}$, experimental studies indicate the importance of electrostatic interactions in determining film and nanoparticle morphology. Many experimental studies have focused on $\mathrm{Au}$ films and NCs with particular emphasis on elucidating the dependence of the morphology on the number of graphene layers. Some interest in these systems is also driven by applications to Surface-Enhanced Raman Spectroscopy (SERS) [217]. A significant number of studies also explore Ag films, focusing on the variation of key features with the number of graphene layers. Various other metals have been considered, with a rather detailed analysis of the filamentary morphologies of $\mathrm{Yb}$ again associated with electrostatic effects.

For metal nanostructures on metal-supported monolayer graphene, the rumpled structure of graphene supported on several single-crystal transition metal surfaces provides a natural, periodically-templated substrate with the potential for directed-assembly of metal NCs at specific locations. Indeed, it is often observed that NCs nucleate and remain in a specific region of the Moiré cell, thereby creating a periodic array of NCs in the regime where all cells are populated. While the basic features of nucleation in the case of significant inter-Moire cell transport are similar to those on an unstructured substrate, the detailed dependence on the various energetic parameters characterizing the interaction of metal adatoms with the rumpled graphene sheet is less clear. There is reason to believe that a simple mean-field treatment of nucleation might not be adequate. For this reason, we 
have implemented atomistic simulations of the nucleation process which by comparison with experiment can more reliably assess these various energetic parameters. We have extended previous modeling to compare energetic parameters for $\mathrm{Ru}, \mathrm{Pt}$, and $\mathrm{Rh}$ on $\mathrm{G} 1 / \mathrm{Ru}(0001$ ) (where the filling factor, FF, or island density decreases, and thus the effective surface mobility increases, from $\mathrm{Ru}$ to $\mathrm{Pt}$ to $\mathrm{Rh}$ ). The behavior of FF versus coverage is naturally compared with the classic case of Ir on G1/Ir(111) where there is negligible inter-moiré cell transport. We have also discussed the extension of experiment and modeling to the case of co-deposition and directed-assembly of bimetallic NCs.

First-principles calculations on the adsorption properties of metal adatom on graphene have provided very useful insights into the growth morphology and thermal stability of metal nanostructures. In particular, the calculations suggest that the 3-D growth mode observed for most metals can be predicted from $\mathrm{E}_{\mathrm{a}} / \mathrm{E}_{\mathrm{b}}$, the ratio between the adsorption energy and the bulk binding energy of the metals. Small $\mathrm{E}_{\mathrm{a}} / \mathrm{E}_{\mathrm{b}}$ ratios favor 3-D morphology and vice versa. In order to form good metal/graphene interfaces through layer-by-layer growth (an essential requirement for good metal contacts in graphene devices), a metal with strong interaction with graphene may be needed. The calculations also show that metals forming large $\mathrm{NCs}$ on graphene (e.g., $\mathrm{Pb}$ and $\mathrm{Eu}$ ) have relatively small diffusion barriers, consistent with classical nucleation theory. The calculations also indicate that metal adsorption can induce substantial changes in the structural, electronic and magnetic properties of graphene which may also affect the growth morphology and stability of the metal NCs, but the detailed mechanisms are still not fully understood.

We note that metal nucleation and growth on graphene and on graphite exhibit quantitatively different behavior. Experiments on exfoliated graphene of variable thickness (ranging from single to multilayer graphene) have shown that the nucleated NC density decreases monotonically with graphene layer thickness. This dependence can be related to the decreasing contribution of the repulsive interactions within the charged metal NCs for growth on multilayer graphene, which favors larger NC size and lower NC density with increasing layer thickness. However, controlling the graphene thickness may provide an interesting avenue to tune metal nucleation so the metal morphology can meet the requirements of various applications. These areas are still in the early stages of investigation.

Finally, we show that intercalation offers an interesting avenue to tune metal nucleation so that the metal growth morphology on graphene can meet the requirements of various applications. These issues are still in the early stages of investigation [218].

\section{Acknowledgements}

Work at Ames Laboratory was supported by the US Department of Energy, Basic Energy Sciences, Division of Materials Science and Engineering, including a grant of computer time at the National Energy Research Scientific Computing Centre (NERSC) in Berkeley, CA under Contract No. DE-AC02-07CH11358. YH and JE were supported for the analysis of metal NCs on metal-supported graphene by NSF Grant CHE-1111500 and DMR-1504593. AKE and RJB were supported by the Baden-Württemburg-Stiftung via the Competence Network "Functional Nanostructures" and by the DFG via the Research Group 1376 (Be 1201/18-1). AKE acknowledges a fellowship from the "Fond national de la recherche" Luxembourg (PHD09-13). Xiaojie Liu also acknowledges the support by 
the National Natural Science Foundation of China under Grant No. 11204013and the China Postdoctoral Science Foundation under Grant No. 2013 T60056.

\section{References}

[1] K.S. Novoselov, A.K. Geim, S.V. Morozov, D. Jiang, Y. Zhang, S.V. Dubonoset, I.V. Grigorieva, A.A. Firsov, Electric field effect in atomically thin carbon films, Science 306 (2004) 666-669.

[2] A.K. Geim, Graphene: Status and Prospects. Science 324 (2009) 1530-1534.

[3] Y. Zhang, Y.-W. Tan, H.L. Stormer, and P. Kim, Experimental observation of the quantum hall effect and Berry's phase in graphene, Nature 438 (2005) 201-204.

[4] R. Zan, U. Bangert, Q. Ramasse, K.S. Novoselov, Metal-Graphene Interaction Studied via Atomic Resolutions Scanning Transmission Electron Microscopy, Nano Letters 11 (2011) 1087-1092.

[5] I. Gierz, C. Riedl, U. Starke, C.R. Ast, K. Kern, Atomic Hole Doping of Graphene, Nano Letters 8 (2008) 4603-4607.

[6] G. Giovannetti, P.A., Khomyakov, G. Brocks, V.M. Karpan, J. van den Brink, and P.J. Kelly, Doping Graphene with Metal Contacts, Phys. Rev. Lett. 101 (2008) 026803.

[7] V.M. Karpan, G. Giovannetti, P.A. Khomyakov, M. Talanana, A.A. Starikov, M. Zwierzycki, J. van den Brink, G. Brocks, P.J. Kelly,Graphite and Graphene as Perfect Spin Filters, Phys. Rev. Lett. 99 (2007) 176602.

[8] C. Vo-Van, Z. Kassir-Bodon, H. Yang, J. Coraux, J. Vogel, S. Pizzini, P. Bayle-Guillemaud, M. Chshiev, L. Ranno, V. Guisset, P. David, V. Salvador, and O. Fruchart, Ultrathin epitaxial cobalt films on graphene for spintronic investigations and applications, New J. Phys. 12 (2010) 103040.

[9] B. Uchoa, T.G. Rappoport, and A.H. Castro Neto, Kondo Quantum Criticality of Magnetic Adatoms in Graphene, Phys. Rev. Lett. 106 (2011) 016801.

[10] H. Sevinçli, M. Topsakal, E. Durgun, S. Ciraci, Electronic and magnetic properties of $3 \mathrm{~d}$ transition-metal atom adsorbed graphene and graphene nanoribbons, Phys. Rev. B 77 (2008) 195434.

[11] J.L. McChesney, A. Bostwick, T. Ohta, T. Seyller, K. Horn, J. González, and E. Rotenberg, Extended van Hove Singularity and Superconducting Instability in Doped Graphene, Phys. Rev. Lett. 104 (2010) 136803.

[12] S. Barraza-Lopez, M. Vanevi , M. Kindermann, and M.Y. Chou, Effects of Metallic Contacts on Electron Transport through Graphene, Phys. Rev. Lett. 104 (2010) 076807.

[13] M. Hupalo, E.H. Conrad, and M.C. Tringides, Growth mechanism for epitaxial graphene on vicinal $6 \mathrm{H}-\mathrm{SiC}(0001)$ surfaces: A scanning tunneling microscopy study, Phys. Rev. B 80 (2009) 041401(R).

[14] J. Hass, W.A. De Heer, E.H. Conrad, The growth and morphology of epitaxial multilayer graphene, J. Phys.:Conden. Matter 20 (2008) 323202.

[15] M. Hupalo, X. Liu, C.Z. Wang, W.C. Lu, Y.X. Yao, K.M. Ho, and M.C. Tringides, Metal nanostructure formation on graphene: Weak versus strong bonding, Adv. Mater. 23 (2011) 2082-2087.

[16] Z. Luo, L. A. Somers, Y. Dan, Thomas Ly, N. J. Kybert, E. J. Mele, and A. T. Charlie Johnson, Size-Selective Nanoparticle Growth on Few-Layer Graphene Films, Nano Letters 10 (2010) 777-781. 
[17] H. Zhou, C. Qiu, Z. Liu, H. Yang, L. Hu, J. Liu, H. Yang, C. Gu, and L. Sun, Thickness-Dependent Morphologies of Gold on N-layer Graphenes, J. Am. Chem. Soc. 132 (2010) 944-946.

[18] L. A. Somers, N. A. Zimbovskaya, A. T. Johnson, and E. J. Mele, Nanoparticle shape selection by repulsive interactions: Metal islands on few-layer graphene, Phys. Rev. B 82 (2010) 115430.

[19] J. Lee, K. S. Novoselov, and H. Suk Shin, Interaction between Metal and Graphene: Dependence on the Layer Number of Graphene, ACS Nano 5 (2011) 608-612.

[20] H. Zhou, C. Qiu, F. Yu, H. Yang, M. Chen, L. Hu, and L. Sun, Thickness-Dependent Morphologies and Surface-Enhanced Raman Scattering of Ag Deposited on n-Layer Graphenes, J. Phys. Chem. C 115 (2011) 11348-11354.

[21] H.-Q. Zhou, H.-C. Yang, C.-Y. Qiu, Z. Liu, F. Yu, L.-J. Hu, X.-X. Xia, H.-F. Yang, C.-Z. Gu, and L.-F. Sun, Aggregation of ferromagnetic and paramagnetic atoms at edges of graphenes and graphite, Chin. Phys. B 20 (2011) 026803.

[22] H.-Q. Zhou, F. Yu, H.-C. Yang, M.-J. Chen, G. Wang, L.-F.Sun, High-throughput thickness determination of n-layer graphenes via gold deposition, Chem. Phys. Lett. 518 (2011) 76-80.

[23] H. Zhou, F. Yu, H. Yang, C. Qiu, M. Chen, L. Hu, Y. Guo, H. Yang, C. Gu and L. Sun, Layer-dependent morphologies and charge transfer of Pd on n-layer graphenes, Chem. Commun. 47 (2011) 9408-9410.

[24] C.-W. Huang, H.-Y. Lin, C.-H. Huang, R.-J. Shiue, W.-H. Wang, C.-Y. Liu1, and H.-C. Chui, Layer-dependent morphologies of silver on n-layer graphene, Nanoscale Research Letters 7 (2012) 618.

[25] L. Liu, Z. Chen, L. Wang, E. Polyakova (Stolyarova), T. Taniguchi, K. Watanabe, J. Hone, G. W. Flynn, and L. E. Brus, Slow Gold Adatom Diffusion on Graphene: Effect of Silicon Dioxide and Hexagonal Boron Nitride Substrates, J. Phys. Chem. B 117 (2013) 4305-4312.

[26] H. Zhou, F. Yu, M. Chen, C. Qiu, H. Yang, G. Wang, T. Yu, L. Sun, The transformation of a gold film on few-layer graphene to produce either hexagonal or triangular nanoparticles during annealing, Carbon 52 (2013) 379-387.

[27] C. Qiu, H. Zhou, B. Cao, L. Sun, T. Yu, Raman spectroscopy of morphology-controlled deposition of Au on graphene, Carbon 59 (2013) 487-494.

[28] S. Uk Yu, B. Park, Y. Cho, S. Hyun, J. Kon Kim, and K. S. Kim, Simultaneous Visualization of Graphene Grain Boundaries and Wrinkles with Structural Information by Gold Deposition, ACS Nano 8 (2014) 8662-8668.

[29] S.-H. Lee, E.-H. Kwak, G.-H. Jeong, Dewetting behavior of electron-beam-deposited $\mathrm{Au}$ thin films on various substrates: graphenes, quartz, and $\mathrm{SiO}_{2}$ wafers, Appl. Phys. A 118 (2015) 389-396.

[30] Y. Qin, J. Han, G. Guo, Y. Du, Z. Li, Y. Song, L. Pi, X. Wang, X. Wan, M. Han and F. Song, Enhanced quantum coherence in graphene caused by Pd cluster deposition, Appl. Phys. Lett. 106 (2015) 023108.

[31] T.P. Hardcastle, C.R. Seabourne, R. Zan, R.M.D. Brydson, U. Bangert, Q.M. Ramasse, K.S. Novoselov, A.J. Scott, Mobile metal adatoms on single layer, bilayer, and trilayer graphene: An ab initio DFT study with van der Waals corrections correlated with electron microscopy data, Phys. Rev. B 87 (2013) 195430.

[32] E.L. Evans, O.P. Bahl, J.M. Thomas, The decoration of, and epitaxial growth of gold on, graphite surfaces, Carbon 5 (1967) 587-588. 
[33] T. Filleter, K. V. Emtsev, Th. Seyller and R. Bennewitz, Local work function measurements of epitaxial graphene, Appl. Phys. Lett. 93 (2008) 133117.

[34] S. S. Datta, D. R. Strachan, E. J. Mele and A. T. Charlie Johnson, Surface Potentials and Layer Charge Distributions in Few-Layer Graphene Films, Nano Letters 9 (2009) 7-11.

[35] M. Batzill, The surface science of graphene: Metal interface, CVD synthesis, nanoribbons, chemical modification, and defect, Surf. Sci. Rep. 67 (2012) 83-115.

[36] X. Li, W. Cai, J. An, S. Kim, J. Nah, D. Yang, R. Piner, A. Velamakanni, I. Jung, E. Tutur, S.K. Banjeree, Sanjay, L. Colombo, R.S. Ruoff, Large-Area Synthesis of High-Quality and Uniform Graphene Films on Copper Foils, Science 324 (2009) 1312-1314.

[37] S. Nie, J.M. Wofford, N.C. Bartelt, O.D. Dubon, K.F. McCarty, Origin of the mosaicity in graphene grown on Cu(111), Phys. Rev. B 84 (2011) 155425.

[38] J. Lahari, Y. Lin, P. Bozkurt, I.I. Oleynik, M. Batzill, An extended defect in graphene as a metallic wire, Nature Nanotechnology 5 (2010) 326-329.

[39] Y.S. Dedkov, M. Fonin, U. Rüdiger, C. Laubschat, Rashba effect in the graphene/Ni(111) system, Phys. Rev. Lett. 100 (2008) 107602.

[40] A. Varykhalov, J. Sánchez-Barriga, A.M. Biswas, E. Vescovo, A. Rybkin, D. Marchenko, O. Rader, Electronic and magnetic properties of quasifreestanding graphene on Ni, Phys. Rev. Lett. 101 (2008) 157601.

[41] J.M. Wofford, E. Starodub, A.L. Walter, S. Nie, A. Bostwick, N.C. Bartelt, K. Thürmer, E. Rotenberg, K.F. McCarty, O.D. Dubon, Extraordinary epitaxial alignment of graphene islands on Au(111), New J. Phys.14 (2012) 053008.

[42] S. Marchini, S. Günther, J. Wintterlin, Scanning tunneling microscopy of graphene on Ru(0001), Phys. Rev. B 76 (2007) 075429.

[43] A.L. Vázquez de Parga, F. Calleja, B. Borca, M.C. Passeggi, J.J. Hinarejos, F. Guinea, R. Miranda, Periodically Rippled Graphene: Growth and Spatially Resolved Electronic Structure, Phys. Rev. Lett. 100 (2008) 056807.

[44] B. Wang, S. Gunther, J. Wintterlin, M.-L.Bocquet, Periodicity, work function and reactivity of graphene on $\mathrm{Ru}(0001)$ from first principles, New J. Phys. 12 (2010) 043401. [45] Y. Pan, H. Zhang, D. Shi, J. Sun, S. Du, F. Liu, H.-J. Gao, Highly Ordered, Millimeter-Scale, Continuous, Single-Crystalline Graphene Monolayer Formed on $\mathrm{Ru}(0001)$,Adv. Mater. 21 (2009) 2777-2780.

[46] W. Moritz, B. Wang, M.-L.Bocquet, T. Brugger, T. Greber, J. Wintterlin, S. Günther, Structure Determination of the Coincidence Phase of Graphene on Ru(0001), Phys. Rev. Lett. 104 (2010) 136102.

[47] B. Borca, S. Barja, M. Garnica, M. Minniti, A. Politano, J.M. Rodriguez-García, J.J. Hinarejos, D. Farías, A.L. Vázquez de Parga, R. Miranda, Electronic and geometric corrugation of periodically rippled, self-nanostructured graphene epitaxially grown on Ru(0001),New J. Phys. 12 (2010) 093018.

[48] D. Stradi, S. Barja, C. Díaz, M. Garnica, B. Borca, J.J. Hinarejos, D. Sánchez-Portal, M. Alcamí, A. Arnau, A.L. Vázquez de Parga, R. Miranda, F. Martín, Role of Dispersion Forces in the Structure of Graphene Monolayers on Ru Surfaces, Phys. Rev. Lett. 106 (2011) 186102.

[49] A.T. N'Diaye, S. Bleikamp, P.J. Feibelman, T. Michely, Two-Dimensional Ir Cluster Lattice on a Graphene Moiré on Ir(111), Phys. Rev. Lett. 97 (2006) 215501.

[50] J. Coraux, A.T. N'Diaye, C. Busse, T. Michely, Structural Coherency of Graphene on $\operatorname{Ir}(111)$, Nano Letters 8 (2008) 565-570. 
[51] J. Coraux, T.N. Plasa, C. Busse, T. Michely, Structure of epitaxial graphene on Ir(111), New J. Phys. 10 (2008) 043033.

[52] C. Virojanadara, S. Watcharinyanon, A.A. Zakharov, and L.I. Johansson, Epitaxial graphene on 6H-SiC and Li intercalation, Phys. Rev. B 82 (2010) 205402.

[53] A. Sandin, T. Jayasekera, J.E. Rowe, K.W. Kim, M.B. Nardelli, and D.B. Dougherty, Multiple coexisting intercalation structures of sodium in epitaxial graphene-SiC interfaces, Phys. Rev. B 85 (2012) 125410.

[54] C.-L. Song, B. Sun, Y.-L. Wang, Y.-P. Jiang, L. Wang, K. He, X. Chen, P. Zhang, X.-C. Ma, Q.-K. Xue, Charge-Transfer-Induced Cesium Superlattices on Graphene, Phys. Rev. Lett. 108 (2012) 156803.

[55] J. Renard, M.B. Lundeberg, J.A. Folk, and Y. Pennec, Real-Time Imaging of K atoms on Graphite: Interactions and Diffusion, Phys. Rev. Lett. 106 (2011) 156101.

[56] K.T. Chan, J. B.Neaton, and M.L. Cohen, First-principles study of metal adatom adsorption on graphene, Phys. Rev. B 77 (2008) 235430.

[57] X. Liu, C.Z. Wang, Y.X. Yao, W.C. Lu, M. Hupalo, M.C. Tringides, and K.M. Ho, Bonding and charge transfer by metal adatom adsorption on graphene, Phys. Rev. B 83 (2011) 235411.

[58] X. Liu, C.Z. Wang, M. Hupalo, H.-Q. Lin, K.M. Ho, M.C. Tringides, Metals on Graphene: Interactions, Growth Morphology, and Thermal Stability, Crystals 3 (2013) 79-111.

[59] X. Liu, C.Z. Wang, M. Hupalo, W.C. Lu, M.C. Tringides, Y.X. Yao, and K.M. Ho, Metals on graphene: correlation between adatom adsorption behavior and growth morphology, Phys. Chem. Chem. Phys. 14 (2012) 9157-9166.

[60] X. Fan, W.T. Zheng, J.-L. Kuo, Adsorption and Diffusion of Li on Pristine and Defective Graphene, ACS Appl. Mater. Interfaces, 4 (2012) 2432-2438.

[61] K.-H. Jin, S.-.M. Choi, S.-H. Jhi, Crossover in the adsorption properties of alkali metals on graphene, Phys. Rev. B 82 (2010) 033414.

[62] C. Uthaisar V. Barone, J. E. Peralta, Lithium adsorption on zigzag graphene nanoribbons, J. Appl. Phys. 106 (2009) 113715.

[63] K. Rytkönen, J.Akola, M. Manninen, Density functional study of alkali-metal atoms and monolayers on graphite (0001), Phys. Rev. B 75 (2007) 075401.

[64] X. Fan, W.T. Zheng, J.-L. Kuo, D.J. Singh, Adsorption of Single Li and the Formation of Small Li Clusters on Graphene for the Anode of Lithium-Ion Batteries, ACS Appl. Mater. Interfaces, 5 (2013), 7793-7797.

[65] M. Khantha, N.A. Cordero, L.M. Molina, J.A. Alonso, L.A. Girifalco, Interaction of lithium with graphene: An ab initio study, Phys. Rev. B 70 (2004) 125422.

[66] A. Lugo-Solis, I. Vasiliev, Ab initio study of K adsorption on graphene and carbon nanotubes: Role of long-range ionic forces, Phys. Rev. B 76 (2007) 235431.

[67] Y.X. Yao, C.Z. Wang, K.M. Ho, Chemical bonding analysis for solid-state systems using intrinsic oriented quasiatomic minimal-basis-set orbitals, Phys. Rev. B 81 (2010) 235119.

[68] X. Liu, C.-Z. Wang, Hai-Qing Lin, and Kai-Ming Ho, Charge oscillation and interaction between potassium adatoms on graphene by first-principles calculations, Phys. Rev. B 91 (2015) 035415.

[69] T. Gao, Y. Gao, C. Chang, Y. Chen, M. Liu, S. Xie, K. He, X. Ma, Y. Zhang, Z. Liu, Atomic-Scale Morphology and Electronic Structure of Manganese Atomic Layers Underneath Epitaxial Graphene on SiC(0001), ACS Nano 6 (2012) 6562-6568. 
[70] S.M. Binz, M. Hupalo, X. Liu, C.Z. Wang, W.C. Lu, P.A. Thiel, K.M. Ho, E.H. Conrad, M.C. Tringides, High Island Densities and Long Range Repulsive Interactions: Fe on Epitaxial Graphene, Phys. Rev. Lett. 109 (2012) 026103

[71] S.W. Poon, A.T.S. Wee, E.S. Tok, Anomalous scaling behavior of cobalt cluster size distributions on graphite, epitaxial graphene and carbon-rich $(6 \sqrt{3} \times 6 \sqrt{ } 3)$ R $30^{\circ}$, Surf. Sci. 606 (2012) 1586-1593.

[72] L. Henrique de Lima, R. Lander. A. de Siervo, Patterning Quasi-Periodic Co 2D-Clusters underneath Graphene on SiC(0001), Chem. Mater. 26 (2014) 4172-4177.

[73] Z.-J. Wang, Q. Fu, Z. Zhang, X. Bao, Growth and characterization of Au, Ni and Au-Ni nanoclusters on 6H-SiC(0001) carbon nanomesh, Surf. Sci. 606 (2012) 1313-1322.

[74] Z.-J. Wang, A. Dong, M. Wei, Q. Fu, X. Bao, Graphene as a surfactant for metal growth on solid surfaces: Fe on graphene/SiC(0001), Appl. Phys. Lett. 104 (2014) 181604.

[75] Y. Mao, J. Yuan, J. Zhong, Density functional calculation of transition metal adatom adsorption on graphene, J. Phys.:Condens. Matter 20 (2008) 115209.

[76] H. Johll, H.C. Kang, Density functional theory of Fe, Co, and Ni adatoms and dimmers adsorbed on graphene, Phys. Rev. B 79 (2009) 245416.

[77] C. Cao, M. Wu, J. Jiang, H.P. Cheng, Transition metal adatom and dimer adsorbed on graphene: Induced magnetization and electronic structures, Phys. Rev. B 81 (2010) 205424.

[78] J.W. Evans, P.A. Thiel and M.C. Bartelt, Morphological evolution during epitaxial thin film growth: Formation of 2D islands and 3D mounds, Surf. Sci. Rep. 61 (2006) $1-128$.

[79] X. Liu, C.Z. Wang, M. Hupalo, W.C. Lu, P.A. Thiel, K.M. Ho, and M.C. Tringides, $\mathrm{Fe}-\mathrm{Fe}$ adatom interaction and growth morphology on graphene, Phys. Rev. B 84 (2011) 235446.

[80] C. Vo-Van, S. Schumacher, J. Coraux, V. Sessi, O. Fruchart, B.B. Brookes, P. Ohresser, T. Michely, Magnetism of cobalt nanoclusters on graphene on iridium, Appl. Phys. Lett. 99 (2011) 142504.

[81] F. Donati, L. Gragnaniello, A. Cavallin, F.D. Natterer, Q. Dubout, M. Pivetta, F. Patthey, J. Dreiser, C. Piamonteze, S. Rusponi, H. Brune, Tailoring the Magnetism of Co Atoms on Graphene through Substrate Hybridization, Phys. Rev. Lett. 113 (2014) 177201 .

[82] F. Donati, Q. Dubout, G. Autès, F. Patthey, F. Calleja, P. Gambardella, O.V. Yazuev. H. Brune, Magnetic Moment and Anisotropy of Individual Co Atoms on Graphene, Phys. Rev. Lett 111 (2013) 236801.

[83] T. Eelbo, M. Wa niowska, P. Thakur, M. Gyamfi, B. Sachs, T.O. Wehling, S. Forti, U. Starke, C. Tieg, A.I. Lichtenstein, R. Wiesendanger, Adatoms and Clusters of 3d Transition Metals on Graphene: Electronic and Magnetic Configurations, Phys. Rev. Lett. 110 (2013) 136804.

[84] T. Eelbo, M. Wa niowska, M. Gyamfi, S. Forti, U. Starke, R. Wiesendanger, Influence of the degree of decoupling of graphene on the properties of transition metal adatoms, Phys. Rev. B 87 (2013) 205443.

[85] M. Gyamfi, T. Eelbo, M. Wa niowska, T.O. Wehling, S. Forti, U. Starke, A.I. Lichtenstein, M.I. Katsnelson, R. Wiesendanger, Orbital selective coupling between Ni adatoms and graphene Dirac electrons, Phys. Rev. B 85 (2012) 161406 (R). 
[86] Y. Wang, V.W. Brar, A.V. Shytov, Q. Wu, W. Regan, H.-Z. Tsai, A. Zettl, L.S. Levitov, M.F. Crommie, Mapping Dirac quasiparticles near a single Coulomb impurity on graphene, Nature Phys. 8 (2012) 653-657.

[87] X. Liu, C.Z. Wang, M. Hupalo, Y.X. Yao, M.C. Tringides, W.C. Lu, and K.M. Ho, Adsorption and growth morphology of rare-earth metals on graphene studied by ab initio calculations and scanning tunneling microscopy, Phys. Rev. B 82 (2010) 245408.

[88] M. Hupalo, S. Binz, and M.C. Tringides, Strong metal-adatom-substrate interaction of Gd and Fe with graphene, J. Phys.: Condens. Matter 23 (2011) 045005.

[89] M.T. Hershberger, M. Hupalo, P.A. Thiel and M.C. Tringides, Growth of fcc(111) Dy multi-height islands on 6H-SiC(0001) graphene, J. Phys.: Condens. Matter 25(2013) 225005.

[90] X. Liu, M. Hupalo, C.Z. Wang, W.C. Lu, P.A. Thiel, K.M. Ho, and M.C. Tringides, Growth morphology and thermal stability of metal islands on graphene, Phys. Rev. B 86 (2012) 081414(R).

[91] D.F. Förster, T.O. Wehling, S. Schumacher, A. Rosch, T. Michely, Phase coexistence of clusters and islands: Europium on graphene, New J. Phys. 14 (2012) 023022.

[92] T. Michely, M. Hohage, M. Bott, G. Comsa, Inversion of growth speed anisotropy in two dimensions, Phys. Rev. Lett. 70 (1993) 3943-3946.

[93] J. Kotaczkiewicz, E. Bauer, The adsorption of Eu, Gd and Tb on the W(110) surface, Surf. Sci. 175 (1986) 487-507.

[94] H. Li, D. Tian, J. Quinn, Y.S. Li, S.C. Wu, F. Jona, Structural and electronic properties of ultrathin films of Gd, Tb, Dy, Ho, and Er, Phys. Rev. B 45 (1992) 3853.

[95] V.K. Kaul, B.M.S. Bist, O.N. Srivastava, The effect of thickness on the electrical resistivity of dysprosium films, Thin Solid Films 30 (1975) 65-72.

[96] R. Zan, U. Bangert, Q. Ramasse, K.S. Novoselov, Evolution of Gold Nanostructures on Graphene, Small, 7 (2011) 2868-2872.

[97] K. Yagyu, T. Tajiri, A. Kohno, K. Takahashi, H. Tochihara, H. Tomokage, T. Suzuki, Fabrication of a single layer graphene by copper intercalation on a $\mathrm{SiC}(0001)$ surface, Appl. Phys. Lett. 104 (2014) 053115.

[98] Z. Wang, Q. Fu, X. Bao, Effect of Substrate Surface Reconstruction on Interaction with Adsorbates: Pt on 6H-SiC(0001), Langmuir, 26 (2010) 7227-7232.

[99] C. Xia, L.I. Johansson, A.A. Zakharov, L. Hultman, C. Virojanadara, Effects of Al on epitaxial graphene grown on $6 \mathrm{H}-\mathrm{SiC}(0001)$, Materials Research Express 1 (2014) 015606.

[100] M.Amft, B.Sanyal, O.Eriksson, N.V.Skorodumova, Small gold clusters on graphene, their mobility and clustering: A DFT study, J. Phys.:Condens. Matter 23 (2011) 205301.

[101] Y. Tang, Z. Yang, X. Dai, Noble metals induced magnetic properties of graphene, J. Magnetism and Magnetic Materials, 323 (2011) 2441-2447.

[102] W. Gao, J.E. Mueller, J. Anton, Q. Jiang, T. Jacob, Nickel Cluster Growth on Defect Sites of Graphene: A Computational Study, Angew. Chem. Int. Ed, 52 (2013) 14237-14241.

[103] Y. Tang, Z. Yang, X. Dai, Tapping of metal atoms in the defects on graphene, J. Chem. Phys. 135 (2011) 224704.

[104] I. Fampiou, A. Ramasubramaniam, Binding of Pt Nanoclusters to Point Defects in Graphene: Adsorption, Morphology, and Electronic Structure, J. Phys. Chem. C 116 (2012) 6543-6555. 
[105] A.T. N'Diaya, T. Gerber, C. Busse, J. Myslive ek, J. Coraux, T. Michely, A versatile fabrication method for cluster superlattices, New J. Phys. 11 (2009) 103045.

[106] A.T. N'Diaya, S. Bleikamp, P.J. Feibelman, T. Michely, Two-Dimensional Ir Clusters Lattice on a Graphene Moiré on Ir(111), Phys. Rev. Lett. 101 (2008) 219904.

[107] T. Gerber, J. Knudsen, P.J. Feibelman, E. Grånäs, P. Stratmann, K. Schulte, J.N. Andersen, T. Michely, CO-Induced Smoluchowski Ripening of Pt Cluster Arrays on the Graphene/Ir(111) Moiré, ACS Nano 7 (2013) 2020-2031.

[108] D. Franz, S. Runte, C. Busse, S. Schumacher, T. Gerber, T. Michely, M. Mantilla, V. Kilic, J. Zegenhagen, A. Stierle, Atomic Structure and Crystalline Order of Graphene-Supported Ir Nanoparticle Lattices, Phys. Rev. Lett. 110 (2013) 065503.

[109] Q.-Q. Zhuo, Q. Wang, Y.-P. Zhang, D. Zhang, Q.-L. Li, C.-H. Gao, Y.-Q. Sun, L. Ding, Q.-J. Sun, S.-D. Wang, J. Zhong, X.-H. Sun, S.-T. Lee, Transfer-Free Synthesis of Doped and Patterned Graphene Films, ACS Nano 9 (2015) 594-601.

[110] M. Kosaka, S. Takano, K. Hasegawa, S. Noda, Direct synthesis of few- and multi-layer graphene films on dielectric substrates by "etching-precipitation" method, Carbon 82 (2015) 254-263.

[111] G. Wang, Y. Zhao, Y. Deng, M. Chen, L. Tao, X. Fan, W. Huang, H. Yang and L. Sun, Synthesizing graphenes directly on $\mathrm{SiO} / \mathrm{Si}$ in open environments by a dual flame method, RCS Adv. 5 (2015) 16691-16695.

[112] Y.S. Kim, K. Joo, S.-K. Jerng, J.H. Lee, E. Yoon and S.-H. Chun, Direct growth of patterned graphene on $\mathrm{SiO} 2$ substrates without the use of catalysts or lithography, Nanoscale 6 (2014) 10100-10105.

[113] R. Zhang, Z.S. Wang, Z.D. Zhang, Z.G. Dai, L.L. Wang, H. Li, L. Zhou, Y.X. Shang, J. He, D.J. Fu, and J.R. Liu, Direct graphene synthesis on $\mathrm{SiO}_{2} / \mathrm{Si}$ substrate by ion implantation, Appl. Phys. Lett. 102 (2013) 193102.

[114] S.C. Xu, B.Y. Man, S.Z. Jiang, C.S. Chen, C. Yang, M. Liu, X.G. Gao, Z.C. Sun, and C. Zhang, Direct synthesis of graphene on $\mathrm{SiO}_{2}$ substrates by chemical vapor deposition, Cryst. Eng. Comm. 15 (2013) 1840-1844.

[115] H. Bi, S. Sun, F. Huang, X. Xie and M. Jiang, Direct growth of few-layer graphene films on $\mathrm{SiO}_{2}$ substrates and their photovoltaic applications, J. Mat. Chem. 22 (2012) 411-416.

[116] Z. Peng, Z. Yan, Z. Sun, and J.M. Tour, Direct Growth of Bilayer Graphene on $\mathrm{SiO} 2$ Substrates by Carbon Diffusion through Nickel, ACS Nano 5 (2011) 8241-8247.

[117] S.-J. Byun, H. Lim, G.-Y. Shin, T.-H. Han, S. Ho Oh, J.-H. Ahn, H. C. Choi, and T.-W. Lee, Graphenes Converted from Polymers, J. Phys. Chem. Letters 2 (2011) 493-497.

[118] W.-G. Luo, H.-F. Wang, K.-M. Cai, W.-P. Han, P.-H. Tan, P.-A. Hu, K.-Y. Wang, Synthesis of Homogenous Bilayer Graphene on Industrial Cu Foil, Chin. Phys. Lett. 31 (2014) 067202.

[119] I.V. Antonova, S.V. Golod, R.A. Soots, Comparison of various methods for transferring graphene and few layer graphene grown by chemical vapor deposition to an insulating $\mathrm{SiO}_{2} / \mathrm{Si}$ substrate, Semicond. 48 (2014) 804-808.

[120] Y.K. Koh, M.-H. Bae, D.G. Cahill, and E. Pop, Reliably Counting Atomic Planes of Few-Layer Graphene (n > 4), ACS Nano 5 (2011) 269-274.

[121] C.H. Lui, Z. Li, K.F. Mak, E. Cappelluti and T.F. Heinz, Observation of an electrically tunable band gap in trilayer graphene, Nat. Phys. 7 (2011) 944-747.

[122] J.A.Venables, G.D.T. Spiller, and M. Hanbucken, Nucleation and growth of thin films, Rep. Prog.Phys.47 (1984) 399-459. 
[123] A.B. Preobrajenski, M.L. Ng, A.S. Vinogradov, M. Martensson, Controlling graphene corrugation on lattice-mismatched substates, Phys. Rev. B 78 (2008) 073401.

[124] M. Sicot, S. Bouvron, O. Zander, U. Rudiger, Y.S. Dedkov, M. Fonin, Nucleation and growth of nickel nanoclusters on graphene Moiré on Rh(111), Appl. Phys. Lett. 96 (2010) 093115.

[125] P. Sutter, J.T. Sadowski, E. Sutter, Graphene on Pt(111): Growth and substrate interaction, Phys. Rev. B 80 (2009) 245411.

[126] B. Wang, X. Ma, M. Caffio, R. Schaub, W.X. Li, Size-Selective Carbon Nanoclusters as Precursors to the Growth of Epitaxial Graphene, Nano Letters 11 (2011) 424-430.

[127] E. Minniussi, M. Pozzo, A. Baraldi, E. Vesselli, R.R. Zhan, G. Comelli, T.O. Mentes, M.A. Nino, A. Locatelli, S. Lizzit, D. Alfe, Thermal Stability of Corrugated Epitaxial Graphene Grown on Re(0001), Phys. Rev. Lett. 106 (2011) 216101.

[128] D. Eom, D. Prezzi, K.T. Kim, H. Zhao, M. Lefenfeld, S. Xiao, C. Nuckolis, M.S. Hybertson, T.F. Heinz, G.W. Flynn, Structure and Electronic Properties of Graphene Nanoislands on Co(0001), Nano Letters 9 (2009) 2844-2848.

[129] Y. Pan, M. Gao, L. Huang, F. Liu, H.-J. Gao, Directed self-assembly of mono dispersed platinum nanoclusters on graphene Moiré template, Appl. Phys. Lett. 95 (2009) 093106.

[130] K. Donner, P. Jakob, Structural properties and site specific interactions of Pt with the graphene/Ru(0001) moiré overlayer, J. Chem. Phys. 131 (2009) 164701.

[131] H. Zhang, Q. Fu, Y. Cui, D.-L. Tan, X.-H. Bao, Fabrication of metal nanoclusters on graphene grown on $\mathrm{Ru}(0001)$, Chin. Sci. Bull. 54 (2009) 2446-2450.

[132] Z. Zhou, F. Gao, D.W. Goodman, Deposition of metal clusters on single-layer graphene/Ru(0001): Factors that govern cluster growth, Surf. Sci. 604 (2010) L31-L38.

[133] E. Sutter, P. Albrecht, B. Wang, M.L. Bocquet, L. Wu, Y. Zhu, P. Sutter, Arrays of $\mathrm{Ru}$ nanoclusters with narrow size distribution template by monolayer graphene on $\mathrm{Ru}$, Surf. Sci. 605 (2011) 1676-1684.

[134] A. Engstfeld, H. Hoster, R.J. Behm, L. Roelofs, X. Liu, C.-Z. Wang, Y. Han, J.W. Evans, Directed assembly of Ru nanoclusters on $\mathrm{Ru}(0001)$-supported graphene: STM studies and atomistic modeling, Phys. Rev. B86 (2012) 085442.

[135] Y. Han, A.K. Engstfeld, C.Z. Wang, L.D. Roelofs, R.J. Behm, J.W. Evans, Atomistic modeling of $\mathrm{Ru}$ nanocluster formation on graphene/ $\mathrm{Ru}(0001)$ : Thermodynamically versus kinetically directed-assembly, MRS Symp. Proc. 1498 (MRS, Pittsburgh, 2013). DOI: 10.1557/opl.2013.106.

[136] B. Wang, M.-L. Bocquet, S. Marchini, S. Gunther, J. Wintterlin, Chemical origin of a graphene moiré overlayer on Ru(0001),Phys. Chem. Chem. Phys. 10 (2008) 3530-3534. [137] R. Kern, G. Le Lay, J.J. Metois, in Current Topics in Materials Science, Vol. 3, E.Kaldis, editor (North Holland, Amsterdam, 1979).

[138] B. Wang, M.-L. Bocquet, Monolayer Graphene and h-BN on Metal Substrates as Versatile Templates for Metallic Nanoclusters, J. Phys. Chem. Lett. 2 (2011) 2341-2345. [139] Y. Han, A.K. Engstfeld, R.J. Behm, J.W. Evans, Atomistic modeling of the directed-assembly of bimetallic Pt-Ru nanoclusters on $\mathrm{Ru}(0001)$-supported monolayer graphene, J. Chem. Phys. 138 (2013) 134703.

[140] A.K. Engstfeld, S. Beckford, C.D. Lorenz, R.J. Behm, Growth of PtRu clusters on $\mathrm{Ru}(0001)$-supported monolayer graphene films, Chem. Phys. Chem. 13 (2012) 3313-3319. 
[141] Q. Liao, H.J. Zhang, K. Wu, H.Y. Li, S.N. Bao, P. He, Nucleation and growth of mono dispersed cobalt nanoclusters on graphene moiré on $\mathrm{Ru}(0001)$,Nanotechnology 22 (2011) 125303.

[142] O.V. Yazyev, A. Pasquarello, Metal adatoms on graphene and hexagonal boron nitride: Towards rational design of self-assembly templates, Phys. Rev. B 82 (2010) 045407.

[143] Y. Xu, L. Semidey-Flecha, L. Liu, Z. Zhou, D.W. Goodman, Exploring the structure and chemical activity of 2-D gold islands on graphene moiré/Ru(0001), Faraday Disc. 152 (2011) 267-276.

[144] X. Niu, R. Vardavas, R.E. Caflisch, C. Ratsch, Level set simulation of directed self-assembly during epitaxial growth, Phys. Rev. B 74 (2006) 193403.

[145] L. Semidey-Flecha, D. Teng, B.F. Habenicht, D.S. Sholl, Y. Xu, Adsorption and diffusion of the $\mathrm{Rh}$ and $\mathrm{Au}$ adatom on graphene moiré/Ru(0001), J. Chem. Phys. 138 (2013) 184710.

[146] B.F. Habenicht, D. Teng, L. Semidey-Flecha, D.S. Sholl, Y. Xu, Adsorption and diffusion of $4 \mathrm{~d}$ and $5 \mathrm{~d}$ transition metal adatoms on graphene/Ru(0001) and the implications for cluster nucleation, Top. Catal. 57 (2014) 69-79.

[147] L.Z. Zhang, S.X. Du , J.T. Sun, L. Huang, L. Meng, W.Y. Xu , L.D. Pan, Y. Pan, Y.L. Wang, W.A. Hofer and H.-J. Gao, Growth mechanism of metal clusters on a graphene/Ru(0001) template, Adv. Mater. Inter. 1 (2014) 1300104.

[148] Y. Han and J.W. Evans, in preparation.

[149] C. Kittel, Introduction to Solid State Physics, John Wiley \& Son, Inc, 6th edn, p. 55.

[150] Y. Tang, Z. Yang, X. Dai, A theoretical simulation on the catalytic oxidation of CO on Pt/graphene, Phys. Chem. Chem. Phys. 14 (2012) 16566-16572.

[151] G. Kim, Y. Kawazoe, K.-R. Lee, Controlled Catalytic Properties of Platinum Clusters on Strained Graphene, J. Phys. Chem. Lett. 3 (2012) 1989-1996.

[152] R. Siburian, T. Kondo, J. Nakamura, Size Control to a Sub-Nanometer Scale in Platinum Catalysts on Graphene, J. Phys. Chem. C 117 (2013) 3635-3645.

[153] E.J. Yoo, T. Okata, T. Akita, M. Kohyama, J. Nakamura, I. Honma, Enhanced Electrocatalytic Activity of Pt Subnanoclusters on Graphene Nanosheet Surface, Nano Letters 9 (2009) 2255-2259.

[154] X. Liu, L. Li, C. Meng, Y. Han, Palladium Nanoparticles/Defective Graphene Composites as Oxygen Reduction Electrocatalysts: A First-Principles Study, J. Phys. Chem. C 116 (2012) 2710-2719.

[155] M. Zhou, A. Zhang, Z. Dai, C. Zhang, Y.P. Feng, Greatly enhanced adsorption and catalytic activity of Au and Pt clusters on defective graphene, J. Chem. Phys. 132 (2010) 194704.

[156] G. Che, S.J. Li, Y. Su, V. Wang, H. Mizuseki, Y.Kawazoe, Improved Stability and Catalytic Properties of $\mathrm{Au}_{16}$ Cluster Supported on Graphene, J. Phys. Chem. C 115 (2011) 20168-20174.

[157] M. Zhou, A. Zhang, Z. Dai, C. Zhang, Y.P. Feng, Greatly enhanced adsorption and catalytic activity of Au and Pt clusters on defective graphene, J. Chem. Phys. 132 (2010) 194704.

[158] W. Ji, C. Zhang, F. Li, P. Li, P. Wang, M. Ren, M. Yuan, First-principles study of small Pd-Au alloy clusters on graphene, RSC, Adv. 4 (2014) 55781.

[159] W. Zhang, D. Cheng, J. Zhu, Theoretical study of CO catalytic oxidation on free and defective graphene-supported Au-Pd bimetallic clusters, RSC Adv. 4 (2014) 42554. 
[160] D.W. Yuan, C. Liu, Z.R. Liu, Structures and catalytic properties of $\operatorname{Pd}_{m} \operatorname{Au}_{n}(m+n=7)$ bimetallic clusters supported on graphene by first-principles studies, Phys. Lett. A 378 (2014) 408-415.

[161] L. Dong, R.R.S. Gari, Z. Li, M.M. Craig, S. Hou, Graphene-supported platinum and platinum-ruthenium nanoparticles with high electrocatalytic activity for methanol and ethanol oxidation, Carbon 48 (2010) 871-787.

[162] S. Zhang, Y. Shao, H. Liao, J. Liu, I.A. Aksay, G. Yin, Y. Lin, Graphene decorated with PtAu Alloy Nanoparticles: Facile Synthesis and Promising Application for Formic Acid Oxidation, Chem. Mater. 23 (2011) 1079-1081.

[163] R. Wang, Z. Wu, C. Chen, Z. Qin, H. Zhu, G. Wang, H. Wang, C. Wu, W. Dong, W. Fan, J. Wang, Graphene-supported Au-Pd bimetallic nanoparticles with excellent catalytic performance in selective oxidation of methanol to methyl formate, Chem. Commun. 49 (2013) 8250.

[164] G. Wang, X. Shen, J. Yao, J. Park, Graphene nanosheets for enhanced lithium storage in lithium ion batteries, Carbon, 47 (2009) 2049-2053.

[165] D. Pan, S. Wang, B. Zhao, M. Wu, H. Zhang, Y. Wang, Z. Jiao, Li Storage Properties of Disordered Graphene Nanosheets, Chem. Mater. 21 (2009) 3136-3142.

[166] T. Bhardwaj, A. Antic, B. Pavan, V. Barone, B. D. Fahlman, Enhanced Electrochemical Lithium Storage by Graphene Nanoribbons, J. Am. Chem. Soc. 132 (2010) 12556-12558.

[167] Z. Jin, D. Nackashi, W. Lu, C. Kittrell, J.M. Tour, Decoration, Migration, and Aggregation of Palladium Nanoparticles on Graphene Sheet, Chem. Mater. 22 (2010) 5695-5699.

[168] V. Sessi, S. Stepanow, A.N. Rudenko, S. Krotzky, K. Kern, F. Hiebel, P. Mallet, J.-Y. Veuillen, O. Šipr, J. Honolka, N.B. Brookes, Single 3D transition metal atoms on multi-layer graphene systems: electronic configurations, bonding mechanisms and role of the substrate, New J. Phys. 16 (2014) 062001.

[169] H. Valencia, A. Gil, G. Frapper, Trends in the Adsorption of 3d Transition Metal Atoms onto Graphene and Nanotube Surfaces: A DFT Study and Molecular Orbital Analysis, J. Phys. Chem. C 114 (2010) 14141-14153.

[170] T.O. Wehling, A.I. Lichtenstein, M.I. Katsnelson, Transition-metal adatoms on graphene: Influence of local Coulomb interactions on chemical bonding and magnetic moments, Phys. Rev. B 84 (2011) 235110.

[171] A.N. Rudenko, F.J. Keil, M.I. Katsnelson, A.I. Lichtenstein, Adsorption of cobalt on graphene: Electron correlation effects from a quantum chemical perspective, Phys. Rev. B 86 (2012) 075422.

[172] Y. Virgus, W. Purwanto, H. Krakauer, S. Zhang, Ab initio many-body study of cobalt adatoms adsorbed on graphene, Phys. Rev. B 86 (2012) 241406(R).

[173] D. Jacob, G. Kotliar, Orbital selective and tunable Kondo effect of magnetic adatoms on graphene: Correlated electronic structure calculations, Phys. Rev. B 82 (2010) 085423.

[174] Y. Virgus, W. Purwanto, H. Krakauer, S. Zhang, Stability, Energeitcs, and Magnetic States of Cobalt Adatoms on Graphene, Phys. Rev. Lett. 113 (2014) 175502.

[175] X. Liu, C.-Z.Wang, M. Hupalo, H.-Q. Lin, K.-M. Ho, and M. C. Tringides, Growth mode and magnetic properties of Fe on graphene, Phys. Rev. B 90 (2014) 155444.

[176] X. Liu, C.-Z. Wang, H.-Q. Lin, and K.-M. Ho, Enhance magnetic moment for $\mathrm{Mn}_{7}$ cluster on graphene, J Phys. Chem. C 118 (2014) 19123-19128. 
[177] R.C. Longo, J. Carrete, J. Ferrer, L.J. Gallego, Structural, magnetic, and electronic properties of $\mathrm{Ni}_{\mathrm{n}}$ and $\mathrm{Fe}_{\mathrm{n}}$ nanostructures $(\mathrm{n}=1-4)$ adsorbed on zigzag nanoribbons, Phys. Rev. B81 (2010) 115418.

[178] C.D. Porter, D. Stroud, Clustering and magnetic anisotropy of Fe adatoms on graphene, Phys. Rev. B85(2012) 235452.

[179] P. Xu, Y. Yang, S.D. Barber, M.L. Ackerman, J.K. Schoelz, I.A. Kornev, S. Barraza-Lopez, L. Bellaiche, and P.M. Thibado, Giant surface charge density of graphene resolved from scanning tunneling microscopy and first-principles theory, Phys. Rev. B 84 (2011) 161409.

[180] A.K. Geim and K.S. Novoselov, The rise of graphene, Nature Materials 6 (2007) 183-191.

[181] S. Wang, Y. Zhang, N. Abidi, and L. Cabrales, Wettability and Surface Free Energy of Graphene Film, Langmuir 25 (2009)11078-11081.

[182]D. Appy, H. Lei, C.-Z.Wang, M.C. Tringides, D.-J. Liu, J.W. Evans, and P.A. Thiel, Transition metals on the (0001) surface of graphite: Fundamental aspects of adsorption, diffusion, and morphology, Prog. Surf. Sci. 89 (2014) 219-238.

[183] R. Anton and I. Schneidereit, In situ TEM investigations of dendritic growth of Au particles on HOPG, Phys. Rev. B 58 (1998) 13874.

[184] S.W. Poon, J.S. Panb and E.S. Tok, Nucleation and growth of cobalt nanostructures on highly oriented pyrolytic graphite, Phys. Chem. Chem. Phys. 8 (2006) 3326-3334.

[185] D. Appy, H. Lei, Y. Han, C.-Z. Wang, M.C. Tringides, D. Shao, E.J. Kwolek, J.W. Evans, and P.A. Thiel, Determining whether metals nucleate homogeneously on graphite: A case study with copper, Phys. Rev. B 90 (2014) 195406.

[186] K.V. Emtsev, A.A. Zakharov, C. Coletti, S. Forti and U. Starke, Ambipolar doping in quasifree epitaxial graphene on $\mathrm{SiC}(0001)$ controlled by Ge intercalation, Phys. Rev. B 84 (2011) 125423.

[187] J. Baringhaus, A. Stohr, S. Forti, S.A. Krasnikov, A.A. Zakharov, U. Starke and C. Tegenkamp, Bipolar gating of epitaxial graphene by intercalation of Ge, Appl. Phys. Lett. 104 (2014) 261602.

[188] K. Li, X. Feng, W.H. Zhang, Y.B. Ou, L.L. Chen, K. He, L.L. Wang, L.W. Guo, G.D. Liu, Q.K. Xue and X.C. Ma, Superconductivity in Ca-intercalated epitaxial graphene on silicon carbide, Appl. Phys. Lett. 103 (2013) 062601.

[189] R. Decker, J. Brede, N. Atodiresei, V. Caciuc, S. Bluegel and R. Wiesendanger, Atomic-scale magnetism of cobalt-intercalated graphene, Phys. Rev. B 87 (2013) 041403 (R).

[190] E. Sutter, P. Albrecht, F.E. Camino and P. Sutter, Monolayer graphene as ultimate chemical passivation layer for arbitrarily shaped metal surfaces, Carbon 48 (2010) 4414-4420.

[191] S. Chen, L. Brown, M. Levendorf, W. Cai, S.-Y. Ju, J. Edgeworth, X. Li, C.W. Magnuson, A. Velamakanni, R.D. Piner, J. Kang, J. Park and R.S. Ruoff, Oxidation Resistance of Graphene-Coated $\mathrm{Cu}$ and Cu/Ni Alloy, ACS Nano 5 (2011) 1321-1327.

[192] D. Prasai, J.C. Tuberquia, R.R. Harl, G.K. Jennings and K.I. Bolotin, Graphene: Corrosion-Inhibiting Coating, ACS Nano 6 (2012) 1102-1108.

[193] N.T. Kirkland, T. Schiller, N. Medhekar and N. Birbilis, Exploring graphene as a corrosion protection barrier, Corrosion Science 56 (2012) 1-4.

[194] M. Topsakal, H. Sahin and S. Ciraci, Graphene coatings: An efficient protection from oxidation, Phy. Rev. B 85 (2012) 155445. 
[195] Y.S. Dedkov, M. Fonin, U. Rudiger and C. Laubschat, Graphene-protected iron layer on Ni(111), Appl. Phys. Lett. 93 (2008) 022509.

[196] M. S. Dresselhaus and G. Dresselhaus, Intercalation compounds of graphite, Adv. Physics 51 (2002) 1-186.

[197] T. Enoki, M. Suzuki and M. Endo, Graphite Intercalation Compounds and Applications, Oxford University Press, Oxford (2003).

[198] J.C. Chacon-Torres, L. Wirtz and T. Pichler, Raman spectroscopy of graphite intercalation compounds: Charge transfer, strain, and electron-phonon coupling in graphene layers, Physica Status Solidi B-Basic Solid State Physics 251 (2014) 2337-2355.

[199] C.E. Giusca, S.J. Spencer, A.G. Shard, R. Yakimova and O. Kazakova, Exploring graphene formation on the C-terminated face of $\mathrm{SiC}$ by structural, chemical and electrical methods, Carbon 69 (2014) 221-229.

[200] M. Petrovic, I.S. Rakic, S. Runte, C. Busse, J.T. Sadowski, P. Lazic, I. Pletikosic, Z.H. Pan, M. Milun, P. Pervan, N. Atodiresei, R. Brako, D. Sokcevic, T. Valla, T. Michely and M. Kralj, The mechanism of caesium intercalation of graphene, Nature Communications 4 (2013) 2772.

[201] S. Schumacher, D.F. Foerster, M. Roesner, T.O. Wehling and T. Michely, Strain in Epitaxial Graphene Visualized by Intercalation, Phys. Rev. Lett. 110 (2013) 086111.

[202] S. Schumacher, T.O. Wehling, P. Lazic, S. Runte, D.F. Foerster, C. Busse, M. Petrovic, M. Kralj, S. Bluegel, N. Atodiresei, V. Caciuc and T. Michely, The Backside of Graphene: Manipulating Adsorption by Intercalation, Nano Letters 13 (2013) 5013-5019.

[203] X. Liu, C.-Z. Wang, M. Hupalo, H.-Q. Lin, K.-M. Ho, P.A. Thiel, and M.C. Tringides, to be published.

[204] S. Vlaic, A. Kimouche, J. Coraux, B. Santos, A. Locatelli and N. Rougemaille, Cobalt intercalation at the graphene/iridium(111) interface: Influence of rotational domains, wrinkles, and atomic steps, Appl. Phy. Lett. 104 (2014) 101602.

[205] L. Jin, Q. Fu, Y. Yang and X.H. Bao, A comparative study of intercalation mechanism at graphene/Ru(0001) interface, Surf. Sci. 617 (2013) 81-86.

[206] N.R. Gall, E.V. Rut'kov and A.Y. Tontegode, Efficiency of the intercalation of aluminum atoms under a monolayer and submonolayer two-dimensional graphite film on a metal, Semiconductors 36 (2002) 276-281.

[207] E.V. Rut'kov and N.R. Gall, Role of edge atoms of graphene islands on metals in nucleation, growth, alkali metal intercalation, Physics of the Solid State 51 (2009) 1738-1743 .

[208] S. Schumacher, F. Huttmann, M. Petrovic, C. Witt, D.F. Forster, C. Vo-Van, J. Coraux, A.J. Martinez-Galera, V. Sessi, I. Vergara, R. Ruckamp, M. Gruninger, N. Schleheck, F.M.Z. Heringdorf, P. Ohresser, M. Kralj, T.O. Wehling and T. Michely, Europium underneath graphene on $\operatorname{Ir}(111)$ : Intercalation mechanism, magnetism, and band structure, Phys. Rev. B 90 (2014) 235437.

[209] M. Sicot, P. Leicht, A. Zusan, S. Bouvron, O. Zander, M. Weser, Y. S. Dedkov, K. Horn and M. Fonin, Size-Selected Epitaxial Nanoislands Underneath Graphene Moire on Rh(111), ACS Nano 6 (2012) 151-158.

[210] Y. Zhang, Y.F. Zhang, D.L. Ma, Q.Q. Ji, W. Fang, J.P. Shi, T. Gao, M.X. Liu, Y.B. Gao, Y.B. Chen, L.M. Xu and Z.F. Liu, Mn atomic layers under inert covers of graphene and hexagonal boron nitride prepared on Rh(111), Nano Research 6 (2013) 887-896. 
[211] L. Huang, Y. Pan, M. Gao, W. Xu, Y. Que, H. Zhou, Y. Wang, S. Du and H.-J. Gao, Intercalation of metal islands and films at the interface of epitaxially grown graphene and $\mathrm{Ru}(0001)$, Appl. Phys. Lett. 99 (2011) 163107.

[212] D.W. Boukhvalov and M.I. Katsnelson, Destruction of graphene by metal adatoms, Appl. Phys. Lett. 95 (2009) 023109.

[213] Y. Cui, J.F. Gao, L. Jin, J.J. Zhao, D.L. Tan, Q. Fu and X.H. Bao, An Exchange Intercalation Mechanism for the Formation of a Two-Dimensional $\mathrm{Si}$ Structure Underneath Graphene, Nano Research 5 (2012) 352-360.

[214] J. Lahiri and M. Batzill, Graphene destruction by metal-carbide formation: An approach for patterning of metal-supported graphene, Appl. Phys. Lett. 97 (2010) 023102.

[215] J. Lahiri, T.S. Miller, A.J. Ross, L. Adamska, I.I. Oleynik and M. Batzill, Graphene growth and stability at nickel surfaces, New J. Phys. 13 (2011) 025001.

[216] W. Luo, W.H. Doh, Y.T. Law, F. Aweke, A. Ksiazek-Sobieszek, A. Sobieszek, L. Salamacha, K. Skrzypiec, F.L. Normand, A. Machocki, and S. Zafeiratos, Single-Layer Graphene as an Effective Mediator of the Metal-Support Interaction, J. Phys. Chem. Lett. 5 (2014) 1837-1844.

[217] J. Lee, S. Shim, B. Kim, and H. Suk Shin, Surface-Enhanced Raman Scattering of Single- and Few-Layer Graphene by the Deposition of Gold Nanoparticles, Chem. Eur. J 17 (2011) 2381-2387.

[218] S. Sarkar, M.L. Moser, X. Tian, X. Zhang, Y.F. Al-Hadeethi, and R. C. Haddon, Metals on Graphene and Carbon Nanotube Surfaces: From Mobile Atoms to Atomtronics to Bulk Metals to Clusters and Catalysts, Chem. Mater. 2014, 26, 184-195. 


\section{Tables}

Table 1. The adsorption energy $\left(E_{a}, \mathrm{eV}\right)$, diffusion barrier $\left(E_{d}, \mathrm{eV}\right)$, charge transfer ( $\Delta q$, electron), bond order $(\xi)$, dipole moment ( $\mathrm{P}$, Debye), magnetic moment of the adatom/graphene system $\left(\mu, \mu_{\mathrm{B}}\right)$, magnetic moment of isolated atoms $\left(\mu_{0}, \mu_{\mathrm{B}}\right)$, in-plane distortion $\left(\mathrm{d}_{/ /}, \AA\right)$, ratio of adsorption energy to bulk binding energy $\left(E_{a} / E_{b}\right.$, where $E_{b}$ are from Ref. 149), and energy difference between bulk binding energy and adsorption energy $\left(E_{b}-E_{a}, \mathrm{eV}\right)$.

\begin{tabular}{|c|c|c|c|c|c|c|c|c|c|c|c|}
\hline Adatom & Site & $E_{a}$ & $E_{d}$ & $\Delta q$ & $\xi$ & P & $\mu$ & $\mu_{0}$ & $\mathrm{~d}_{/ /}$ & $E_{\mathrm{a}} / E_{b}$ & $E_{b}-E_{\mathrm{a}}$ \\
\hline $\mathrm{Li}$ & $\mathrm{H}$ & 1.2562 & 0.2941 & 1.00 & -0.12 & 3.36 & 0.00 & 1.00 & 0.0178 & 0.7707 & 0.3738 \\
\hline $\mathrm{Na}$ & $\mathrm{H}$ & 0.6716 & 0.1302 & 1.00 & -0.06 & 5.43 & 0.00 & 1.00 & 0.0153 & 0.6034 & 0.4414 \\
\hline K & $\mathrm{H}$ & 1.0880 & 0.0641 & 1.00 & -0.14 & 6.84 & 0.00 & 1.00 & 0.0124 & 1.1649 & -0.1540 \\
\hline Cs & $\mathrm{H}$ & 1.4445 & 0.0342 & $0.90^{a}$ & . & 7.80 & 0.00 & 1.00 & 0.0047 & 1.7966 & -0.6405 \\
\hline $\mathrm{Mg}$ & $\mathrm{H}$ & 0.0279 & 0.0002 & 0.05 & 0.00 & 0.15 & 0.00 & 0.00 & 0.0004 & 0.0185 & 1.4821 \\
\hline $\mathrm{Ca}$ & $\mathrm{H}$ & 0.4704 & 0.1414 & 1.05 & -0.10 & 1.73 & 0.00 & 0.00 & 0.0162 & 0.2557 & 1.3696 \\
\hline $\mathrm{Al}$ & $\mathrm{H}$ & 1.0740 & 0.1414 & 0.88 & 0.41 & 1.14 & 0.00 & 1.00 & 0.0188 & 0.3168 & 2.3160 \\
\hline In & $\mathrm{H}$ & 0.8113 & 0.1088 & 0.68 & 0.46 & 3.08 & 0.00 & 1.00 & 0.0172 & 0.3219 & 1.7087 \\
\hline $\mathrm{Pb}$ & $\mathrm{T}$ & 0.2294 & 0.0011 & 0.50 & 0.72 & 0.86 & 1.74 & 2.00 & 0.0081 & 0.1130 & 1.8007 \\
\hline V & $\mathrm{H}$ & 1.1016 & 0.1961 & 0.57 & 1.43 & 2.17 & 4.50 & 3.00 & 0.0138 & 0.2075 & 4.2084 \\
\hline $\mathrm{Cr}$ & B & 0.1874 & 0.0058 & 0.48 & 0.55 & 0.09 & 5.64 & 6.00 & 0.0066 & 0.0457 & 3.9126 \\
\hline $\mathrm{Mn}$ & $\mathrm{H}$ & 0.1590 & 0.0683 & 0.57 & 0.52 & 0.12 & 5.85 & 5.00 & 0.0096 & 0.0545 & 2.7610 \\
\hline $\mathrm{Fe}$ & $\mathrm{H}$ & 0.8980 & 0.5841 & 1.49 & 1.78 & 2.10 & 1.99 & 4.00 & 0.0213 & 0.2098 & 3.3820 \\
\hline Co & $\mathrm{H}$ & 1.2257 & 0.4151 & 0.97 & 2.02 & 1.61 & 1.00 & 3.00 & 0.0195 & 0.2792 & 3.1643 \\
\hline $\mathrm{Ni}$ & $\mathrm{H}$ & 1.5346 & 0.2167 & 1.23 & 1.47 & 1.15 & 0.00 & 2.00 & 0.0170 & 0.3456 & 2.9054 \\
\hline $\mathrm{Pd}$ & B & 1.0756 & 0.0417 & 0.64 & 1.16 & 1.32 & 0.00 & 0.00 & 0.0116 & 0.2765 & 2.8144 \\
\hline $\mathrm{Pt}$ & B & 1.5516 & 0.1878 & 0.52 & 1.95 & 0.46 & 0.00 & 2.00 & 0.0220 & 0.2657 & 4.2884 \\
\hline $\mathrm{Cu}$ & $\mathrm{T}$ & 0.2273 & 0.0037 & 0.62 & 0.30 & 0.79 & 0.97 & 1.00 & 0.0133 & 0.0913 & 2.2627 \\
\hline $\mathrm{Ag}$ & B & 0.0212 & 0.0038 & 0.01 & 0.00 & 0.39 & 1.00 & 1.00 & 0.0005 & 0.0072 & 2.9288 \\
\hline $\mathrm{Au}$ & $\mathrm{T}$ & 0.0964 & 0.0074 & -0.28 & 0.38 & 1.41 & 1.00 & 1.00 & 0.0095 & 0.0253 & 3.7136 \\
\hline $\mathrm{Nd}$ & $\mathrm{H}$ & 1.9776 & 0.4396 & 0.67 & 1.73 & 3.73 & 2.26 & 1.00 & 0.0132 & 0.5817 & 1.4224 \\
\hline Sm & $\mathrm{H}$ & 1.8165 & 0.3724 & 0.67 & 1.72 & 3.25 & 2.25 & 1.00 & 0.0139 & 0.8488 & 0.3235 \\
\hline $\mathrm{Eu}$ & $\mathrm{H}$ & 0.9975 & 0.1285 & 0.81 & 0.66 & 1.81 & 0.98 & 0.00 & 0.0074 & 0.5363 & 0.8625 \\
\hline $\mathrm{Gd}$ & $\mathrm{H}$ & 1.6607 & 0.3098 & 0.70 & 1.62 & 2.78 & 2.22 & 1.00 & 0.0144 & 0.4011 & 2.4793 \\
\hline Dy & $\mathrm{H}$ & 1.5259 & 0.1773 & 0.73 & 1.75 & 2.47 & 2.19 & 1.00 & 0.0147 & 0.5019 & 1.5141 \\
\hline $\mathrm{Yb}$ & $\mathrm{H}$ & 0.4244 & 0.1496 & 0.85 & 0.10 & 1.61 & 1.01 & 0.00 & 0.0082 & 0.2653 & 1.1756 \\
\hline
\end{tabular}

${ }^{a}$ Charge transfer obtained by Bader analysis; others obtained from QUABMOs methods [67].

Table 2. Comparison between the best available values of: $\mathrm{E}_{\mathrm{a}} / \mathrm{graphene}$ in $\mathrm{eV}$ (Ref. [57]), $\mathrm{E}_{\mathrm{a}} /$ graphite in eV (Ref. [182] except for Cr from Ref. [32]), and $\mathrm{E}_{\mathrm{a}} /$ metals in $\mathrm{eV}$ (from Ref. [148] and Da-Jiang Liu, unpublished). All values come from DFT calculations. All calculations for $\mathrm{E}_{\mathrm{a}} / \mathrm{graphite}$ include dispersion force correction.

\begin{tabular}{cccc}
\hline Adatom & Graphene & Graphite & Metals \\
\hline $\mathrm{Cr}$ & 0.187 & 0.832 & 3.41 \\
$\mathrm{Pt}$ & 1.552 & 1.869 & 4.50 \\
$\mathrm{Cu}$ & 0.227 & 0.512 & 2.27 \\
$\mathrm{Ag}$ & 0.021 & 0.282 & 1.91 \\
$\mathrm{Au}$ & 0.096 & 0.495 & 2.32 \\
\hline
\end{tabular}

Figures 


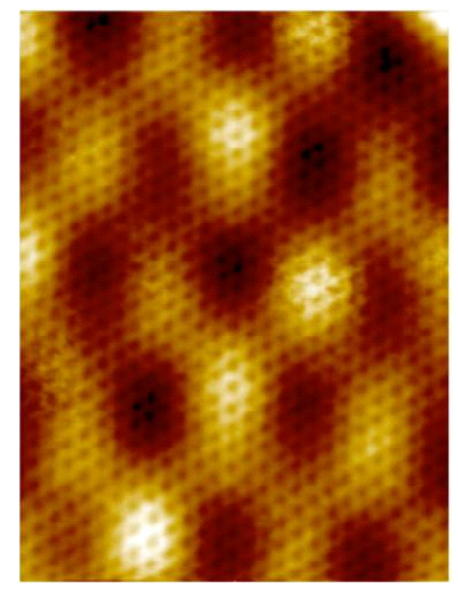

Fig.1. STM image $\left(5.56 \times 7.31 \mathrm{~nm}^{2}\right)$ of the clean graphene lattice supported on Si-terminated SiC (0001) showing corrugation spots due to the $6 \sqrt{ } 3$ reconstruction. The periodicity of the graphene lattice is $0.25 \mathrm{~nm}$ and the periodicity of the corrugation is $1.84 \mathrm{~nm}$ which is close to the size of the $6 \times 6$ supercell that is commonly observed with STM (depending on tunneling voltage). Adapted from Ref. [13]. Copyright @ 2009 American Physical Society.
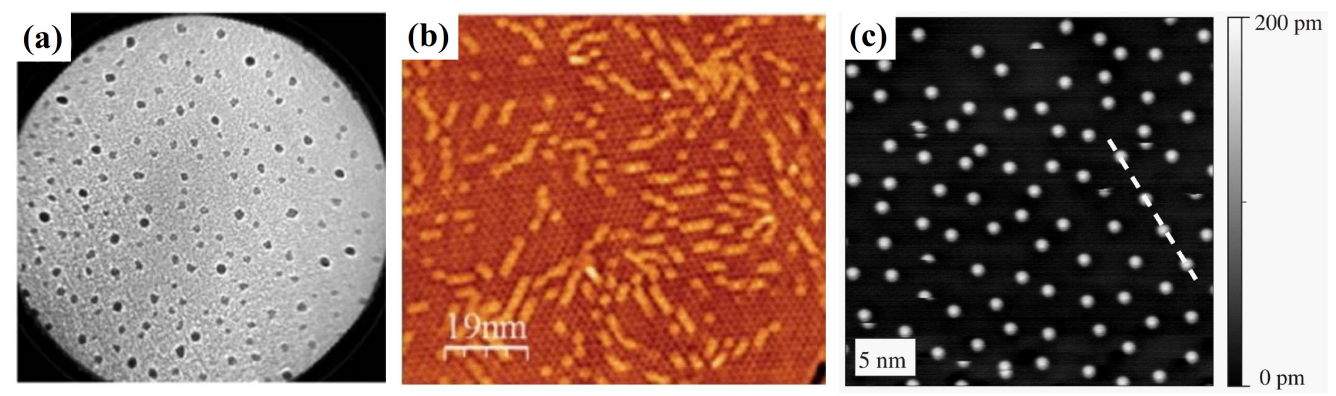

Fig. 2. (a) LEEM image of Li deposition on graphene/SiC(0001). The field of view is 50 $\mu \mathrm{m}$ and the electron energy is $\mathrm{E}_{\mathrm{vac}}-1.7 \mathrm{eV}$. Adapted from Ref. [52]. Copyright @ 2010 American Physical Society. (b) STM image of Low-coverage Na chains (V=-2.18 V, 62 pA) on graphene/SiC(0001). Adapted from Ref. [53]. Copyright @ 2012 American Physical Society. (c) Topographic image of the graphite surface after the deposition of potassium, showing isolated potassium atoms. The tunneling bias and current are $-2 \mathrm{~V}$ and 5pA. Adapted from Ref. [55]. Copyright @ 2011 American Physical Society.
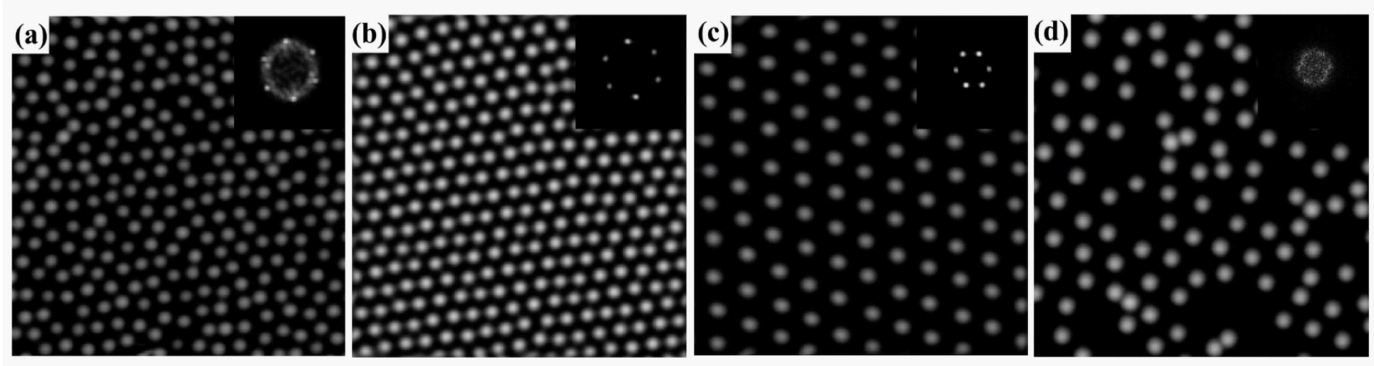
Fig. 3. STM images $\left(30 \times 30 \mathrm{~nm}^{2}\right)$ of Cs on graphene. (a) 1 ML Cs deposition on monolayer graphene. (b) 1 ML Cs deposition on bilayer graphene. (c) 1/3 ML Cs deposition on bilayer graphene. (d) 1/3 ML Cs deposition on six layers graphene. Insets display the corresponding power spectra obtained from Fourier transform of the STM images. Adapted from Ref. [54]. Copyright@ 2012 American Physical Society.

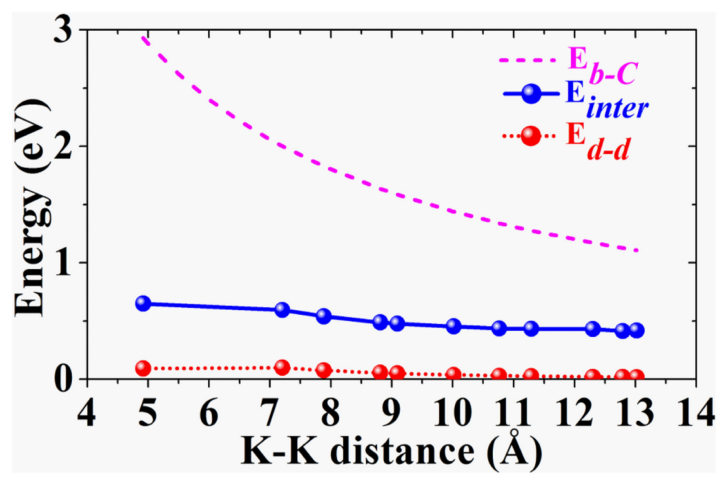

Fig. 4. Interaction energy $\mathrm{E}_{\text {inter }}$ (blue solid circles and blue solid line) of $\mathrm{K}-\mathrm{K}$ adatoms on graphene as a function of K-K separation. The electric dipole-dipole interaction $\mathrm{E}_{d-d}$ (red solid circles and red dot line) and bare Coulomb interaction $\mathrm{E}_{b-c}$ (pink dashed line), respectively, are also shown for comparison. Adapted from Ref. [68]. Copyright @ 2015 American Physical Society.
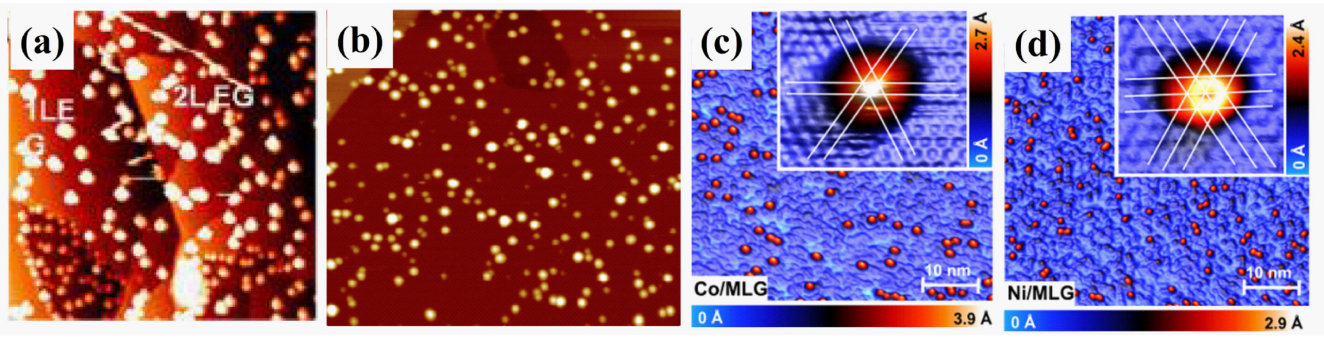

Fig. 5. (a) Mn on graphene: STM image $\left(150 \times 150 \mathrm{~nm}^{2}\right),-0.12 \mathrm{~V}, 0.37 \mathrm{nA}$. Adapted from Ref. [69]. Copyright @ 2012 American Chemical Society. (b) Fe on EG/SiC(0001): STM image $\left(250 \times 220 \mathrm{~nm}^{2}\right), \theta=0.64 \mathrm{ML}, \mathrm{F}=0.054 \mathrm{ML} / \mathrm{min}, \mathrm{RT}$. The NC density is $\mathrm{N}=$ $3.9 \times 10^{-3} \mathrm{NCs} / \mathrm{nm}^{2}$. Adapted from Ref. [88]. Copyright@2011 IOP Publishing Ltd Printed in the UK \& the USA. (c) Co on graphene. The inset exhibits top site adsorption. The tunneling parameters are $\mathrm{U}=0.4(-0.5) \mathrm{V}$ and $\mathrm{I}=0.025(0.3) \mathrm{nA}$. Adapted from Ref. [83]. Copyright@2013 American Physical Society. (d) Ni on graphene. The inset reveals hollow site adsorption. The tunneling parameters are $\mathrm{U}=0.4(-0.1) \mathrm{V}$ and $\mathrm{I}=0.1(0.15) \mathrm{nA}$. Adapted from Ref. [83]. Copyright @ 2013 American Physical Society. 

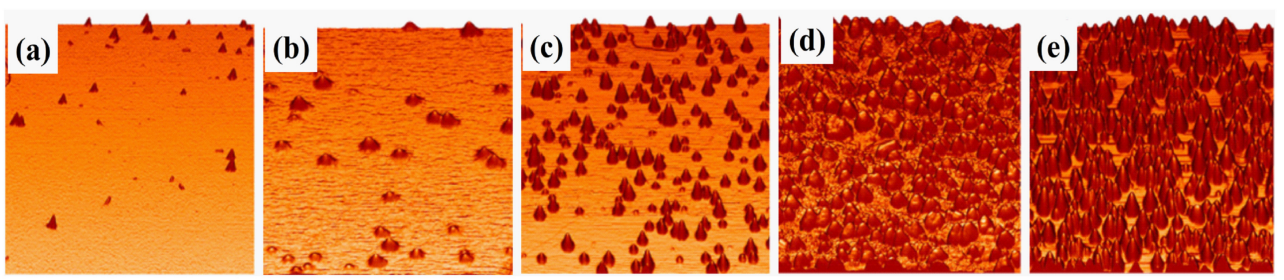

Fig. 6. Fe on graphene. (a) $0.003 \mathrm{ML}$, (b) $0.16 \mathrm{ML}$, (c) $0.65 \mathrm{ML}$, (d) $0.87 \mathrm{ML}$, and (e) 2.3 ML. Small NCs in each image show the new NCs that have just nucleated. All images are $200 \times 200 \mathrm{~nm}^{2}$ except (b) which is $150 \times 150 \mathrm{~nm}^{2}$. Adapted from Ref. [70]. Copyright @ 2012 American Physical Society. 

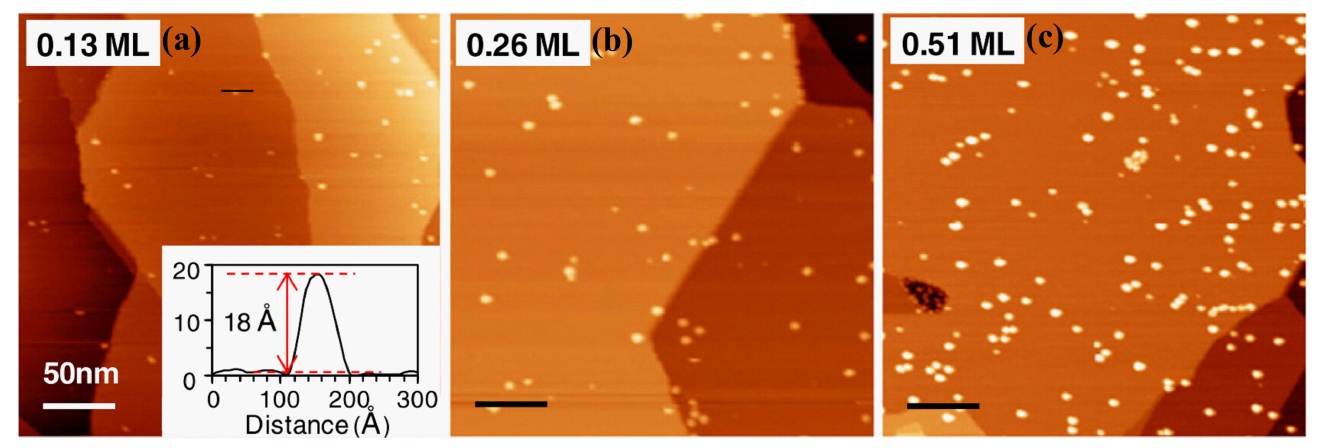

Fig.7. STM images at the Co coverage of (a) $0.13 \mathrm{ML}$, (b) $0.26 \mathrm{ML}$, and (c) $0.51 \mathrm{ML}$ showing morphologies of $\mathrm{Co}$ on $\mathrm{EG} / 6 \sqrt{3} / \mathrm{SiC}$ at room-temperature. Co flux used is $(4.3 \pm 0.2) \times 10^{-3} \mathrm{ML} / \mathrm{s}$. Line profile in (a) showing the cross-section of one of the Co clusters is given as inset. The sample biases used are between -1.3 and $-1.5 \mathrm{~V}$ for images. Adapted from Ref. [71]. Copyright @ 2012 Elsevier B.V. All rights reserved.

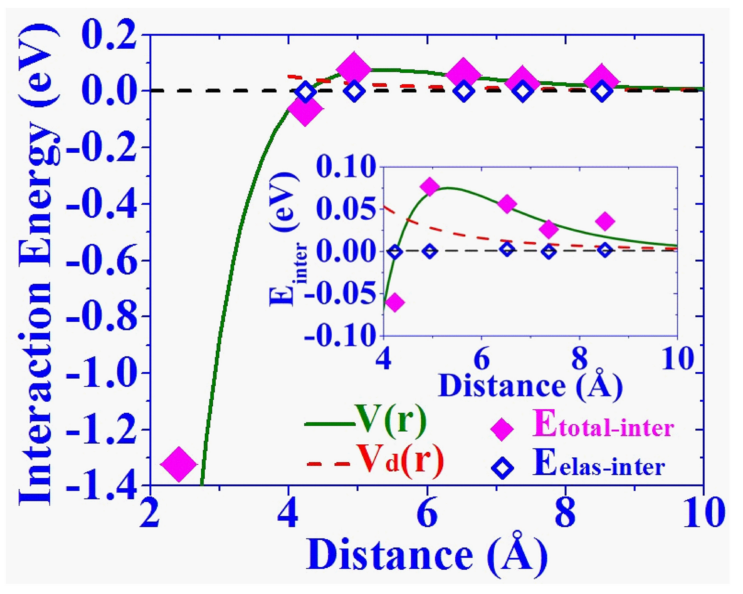

Fig. 8. Interaction energy $\mathrm{E}_{\text {total-inter }}$ (solid diamond) for pairs of Fe adatoms on graphene as a function of Fe-Fe separation $r$ from first-principles DFT calculations. V(r) (solid green line) is fitted to the total interaction energy from the DFT calculations. $V_{d}(r)$ (dashed red line)) is the dipole-dipole interaction energy. The elastic interaction energies from DFT calculations are also shown (open diamond). Adapted from Ref. [79]. Copyright@2011 American Physical Society. 

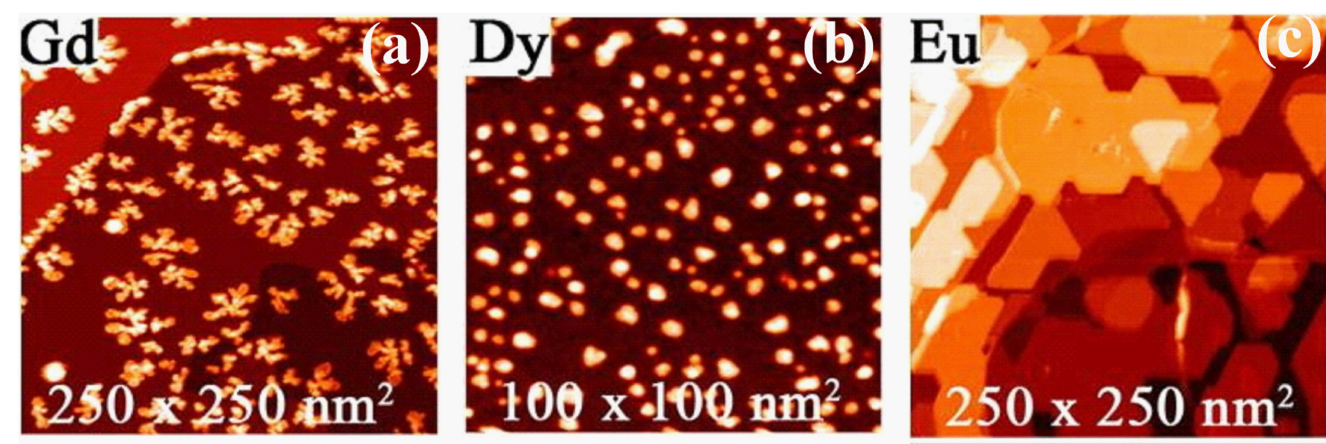

Fig. 9. Growth morphologies of RE on graphene/SiC. (a) High NC density and fractal-shape islands were observed for Gd (Adapted from Ref. [88]. Copyright @ 2011 IOP Publishing Ltd Printed in the UK \& the USA.). (b) High NC density was also observed for Dy. (Adapted from Ref. [89]. Copyright @2013 IOP Publishing Ltd Printed in the UK \& the USA.). (c) layer-by-layer growth was observed for Eu. (Adapted from Ref. [87]. Copyright@ 2010 American Physical Society).
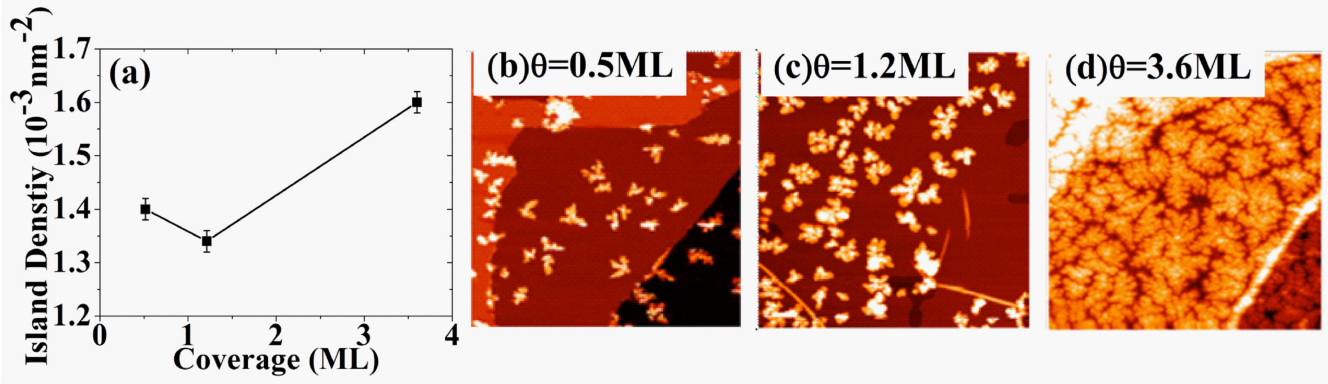

Fig. 10. (a) Practically constant island density vs coverage at room temperature for Gd on graphene with $\mathrm{F}=0.12 \mathrm{ML} / \mathrm{min}$. The Corresponding images are shown in (b)-(d). (b) $210 \times 210 \mathrm{~nm}^{2}, 0.5 \mathrm{ML}$ (c) $250 \times 250 \mathrm{~nm}^{2}, 1.2 \mathrm{ML}$ (d) $250 \times 250 \mathrm{~nm}^{2}, 3.6 \mathrm{ML}$. Adapted from Ref. [88]. Copyright @ 2011 WILEY-VCH Verlag GmbH \& Co. KGaA, Weinheim. 

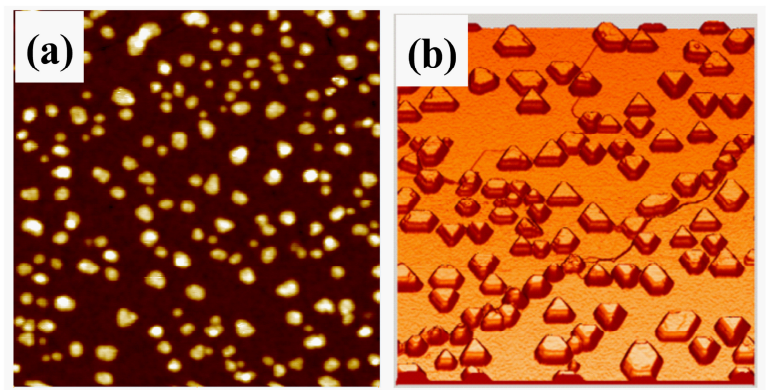

Fig. 11. (a) The nucleated island density after $0.4 \mathrm{ML}$ of Dy has been deposited at room temperature over an area of $1000 \times 1000 \AA^{2}$. The island density is $2 \times 10^{-4}$ islands per $\AA^{2}$ indicating slow diffusion. Adapted from Ref. [15] Copyright @ 2011 WILEY-VCH Verlag GmbH \& Co. KGaA, Weinheim. (b) Dy NCs grown on epitaxial graphene at $660 \mathrm{~K}$ after continuous deposition, with 1.36 ML. The corresponding area is $250 \times 240 \mathrm{~nm}^{2}$. The majority of the NCs have triangular shapes with equal number of NCs pointing in opposite directions suggesting they are fcc(111) NCs. Adapted from Ref. [89]. Copyright@2013 IOP Publishing Ltd Printed in the UK \& the USA.
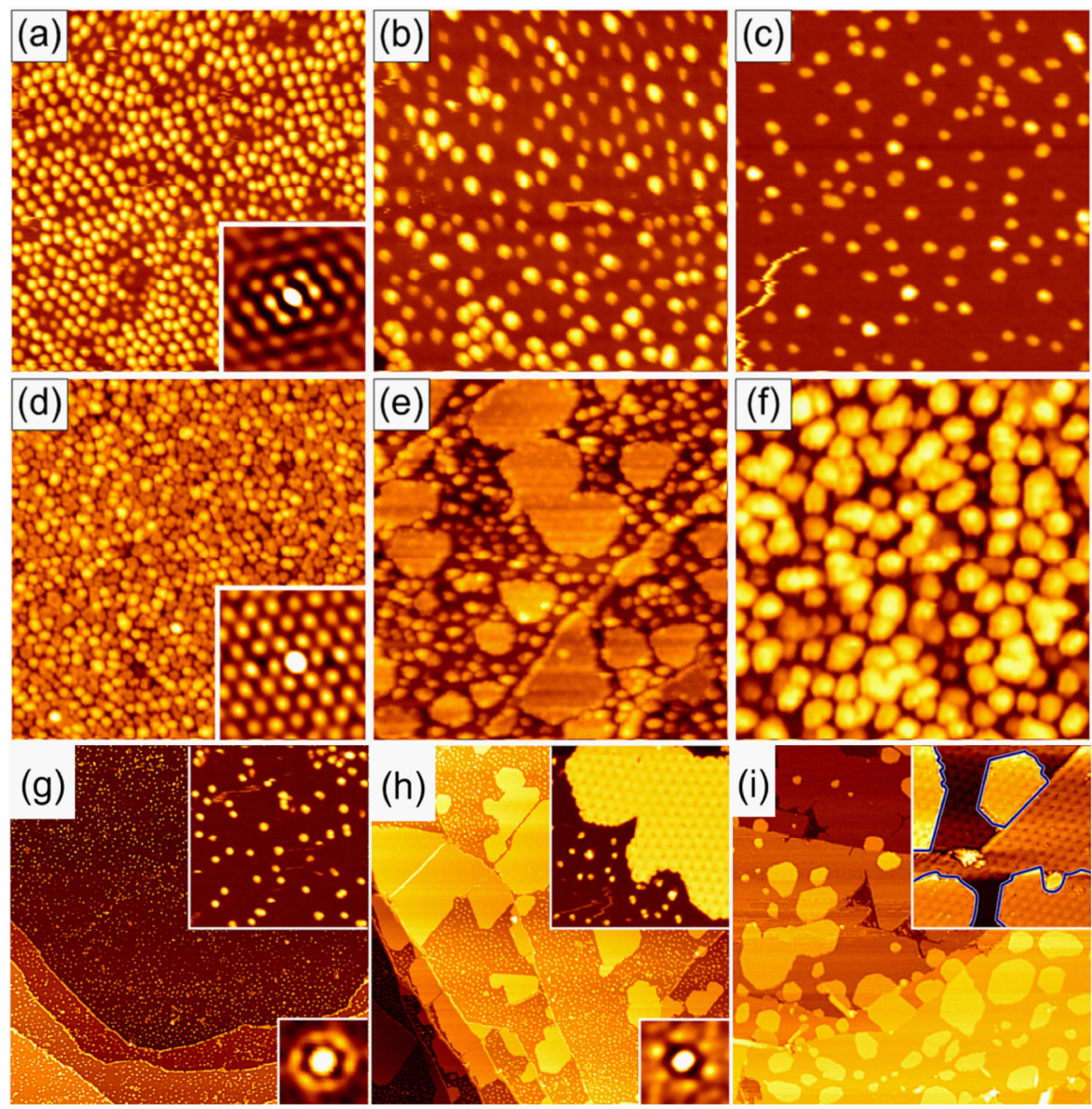

Fig. 12. (a)-(f) STM images of Eu on graphene supported by $\operatorname{Ir}(111)$ substrate at $35 \mathrm{~K}$. (a) $0.008 \mathrm{ML}$, Eu monomers and dimers. (b) 0.018 ML, Eu monomers and dimers. (c) 0.033 
ML, Eu monomers and dimers. (d) 0.05ML, Eu dimers, trimers and tetramers. (e) 0.15 ML, $\mathrm{Eu}_{10}$ cluster. (f) $0.25 \mathrm{ML}, \mathrm{Eu}_{24}$ cluster. (g)-(i) STM images of Eu on graphene supported by $\operatorname{Ir}(111)$ substrate at room temperature, $320 \times 320 \mathrm{~nm}^{2}$ (insets: $40 \times 40 \mathrm{~nm}^{2}$ ). (g) $0.12 \mathrm{ML}$. Inset at the lower right: $2 \mathrm{D}$ self-correlation of a $60 \times 60 \mathrm{~nm}^{2}$ image part covered by clusters only. (h) 0.40 ML. The inset if filtered to enhance the moiré contrast. Adapted from Ref. [91]. Copyright @ IOP Publishing Ltd and Deutsche Physikalische Gesellschaft.
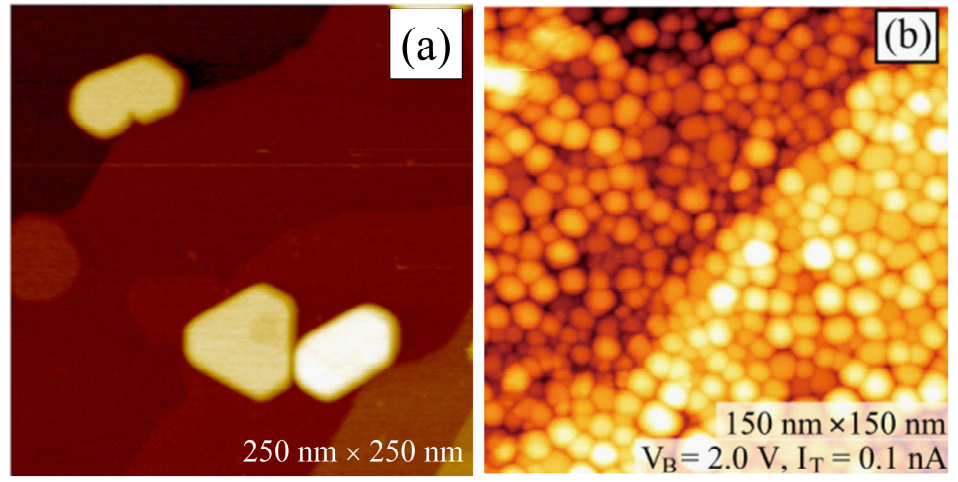

Fig. 13. (a) $\mathrm{Pb}$ on graphene: $250 \times 250 \mathrm{~nm}^{2}$ area with only 3 large $\mathrm{Pb}$ islands the deposition temperature is $40 \mathrm{~K}$ and then annealed to room temperature, deposited amount 0.8 ML. The NCs have heights of 8-, 6-, 7- layers from left to right. Adapted from Ref. [15]. Copyright @ 2011 WILEY-VCH Verlag GmbH \& Co. KGaA, Weinheim. (b) Cu on ZLG (zero layer graphene) after $\mathrm{Cu}$ deposition with the amount of $12 \AA$. Adapted from Ref. [97]. Copyright @2014 AIP Publishing LLC. 

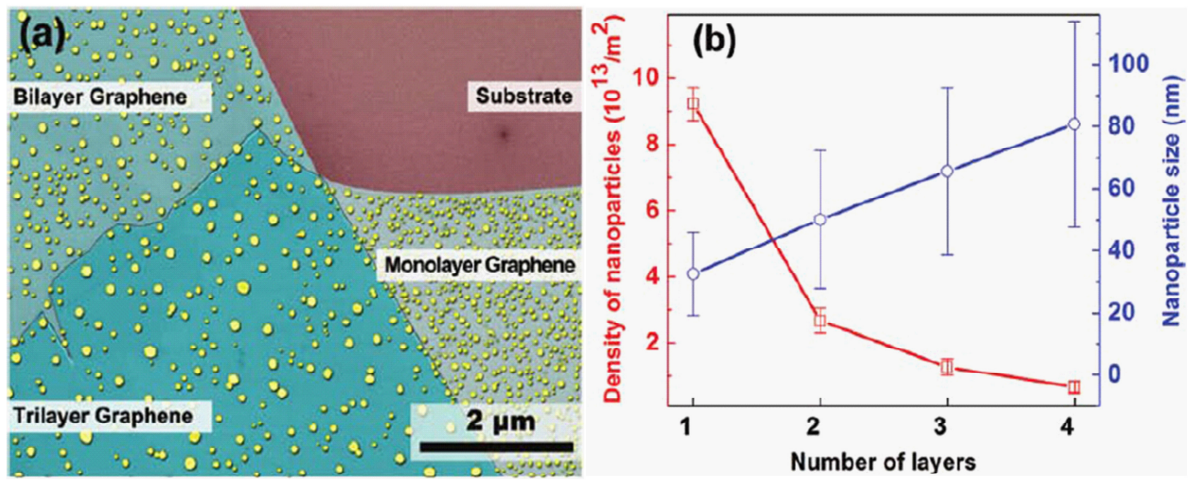

Fig. 14. Morphologies, size, and density of Au NPs on n-layer graphene after annealing at $1535 \mathrm{~K}$ in vacuum for $30 \mathrm{~s}$. No Au NPs are found in the substrate. (a) Au NPs on 1-layer, 2-layer, and 3-layer graphene, respectively. (b) Statistics of the size and density of Au NPs on n-layer graphene. Adapted from Ref. [17]. Copyright @ 2009 American Chemical Society.
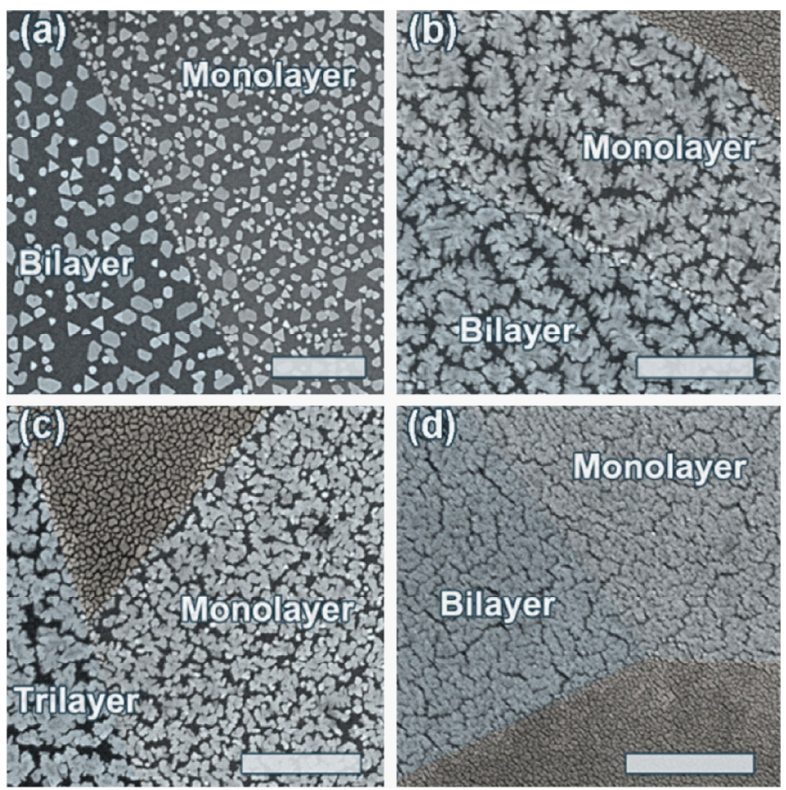

Fig. 15. Typical SEM images of various Au nanostructures formed by deposition of Au on n-layer graphene. Scale bar: $500 \mathrm{~nm}$. (a) Polygons. (b) Dendrites. (c) Irregular islands. (d) Dense clusters. Adapted from Ref. [27]. Copyright @ 2013 Elsevier B.V. All rights reserved. 

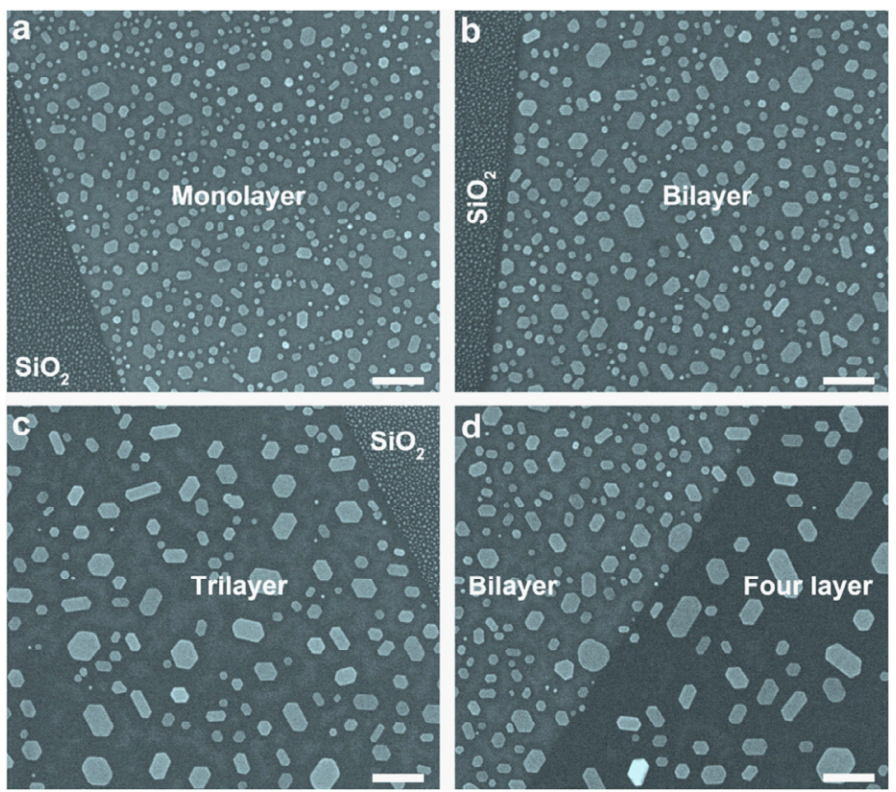

Fig. 16. SEM images showing the transformation of Au nanostructures on graphene into 6-sided polygonal Au NPs after annealing at $975 \mathrm{~K}(\mathrm{a}, \mathrm{c})$ and also to triangular Au NPs annealing at $1075 \mathrm{~K}(\mathrm{~b}, \mathrm{~d})$ for $2 \mathrm{~h}$. Film thickness: $2.0 \mathrm{~nm}$. Scale bar: $200 \mathrm{~nm}$. Adapted from Ref. [26]. Copyright @2013 Elsevier B.V. All rights reserved.
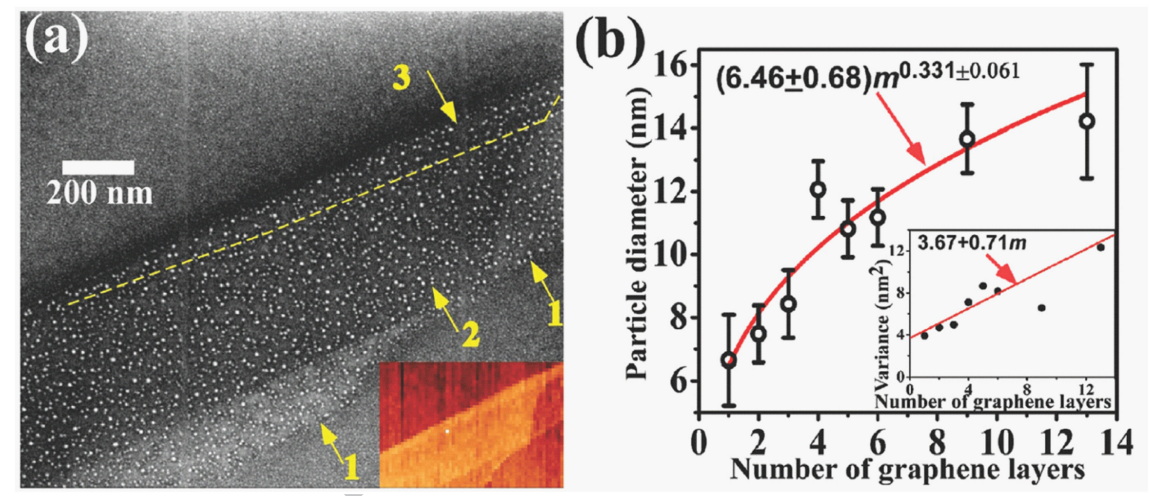

Fig. 17. (a) HRSEM images of Au NPs prepared on n-layer graphene on a $\mathrm{SiO}_{2} / \mathrm{Si}$ substrate. The numbers and arrow indicate the number of graphene layers. Inset in (a) is an AFM image of the same region before nanoparticle growth. (b) Average diameter of Au NPs as a function of $n$. The red line is a power law fit suggested by theory. Inset in (b) is the corresponding dependence of the variance of the diameter distribution; the curve shows the theoretically predicated linear dependence. Adapted from Ref. [16]. Copyright @ 2010 American Chemical Society. 

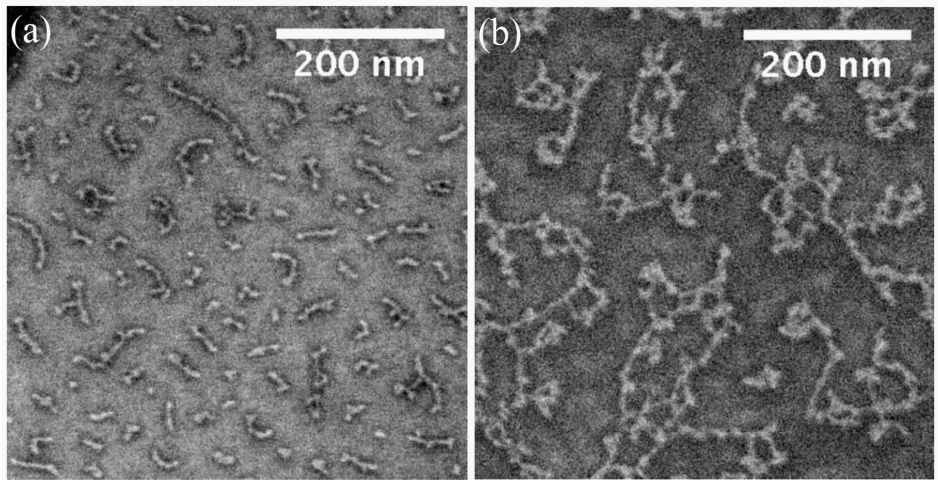

Fig. 18. SEM image of Yb NPs formed after annealing on graphene. The metal atoms condense to form anisotropic filamentary structures. The left panel is for an average coverage of $0.12 \mathrm{~nm}$ and the right is for an average coverage of $0.5 \mathrm{~nm}$. Adapted from Ref. [18]. Copyright @2010 American Physical Society. 

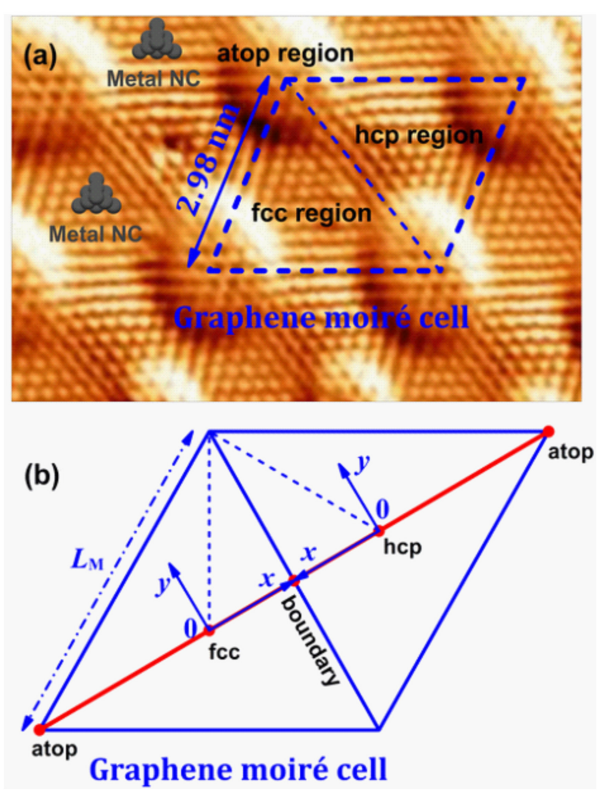

Fig. 19. Schematics showing: (a) periodically-modulated G1-Ru(0001) from an STM image from Ref. [139] indicating one moiré cell and also including superimposed NCs in the fcc region; (b) the coordinate system used to describe the surface energetics in our modeling. Copyright @ 2013 American Institute of Physics.

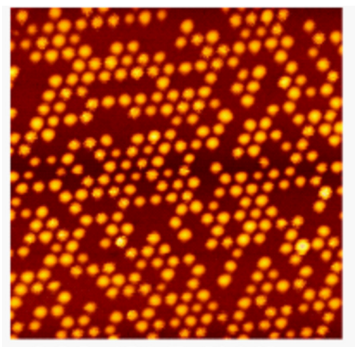

(a) $\operatorname{Ir} / G 1 / \operatorname{Ir}(111)$

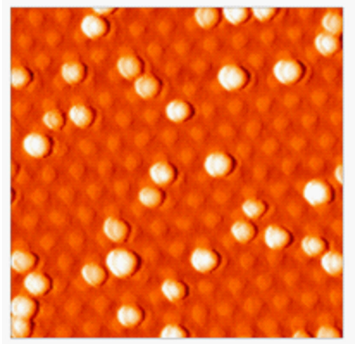

(c) Pt/G1/Ru(0001)

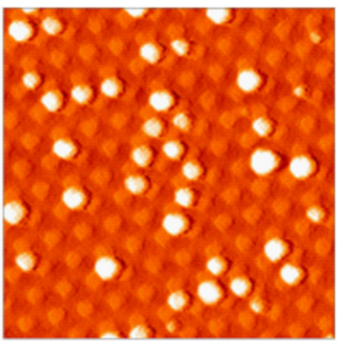

(b) Ru/G1/Ru(0001)

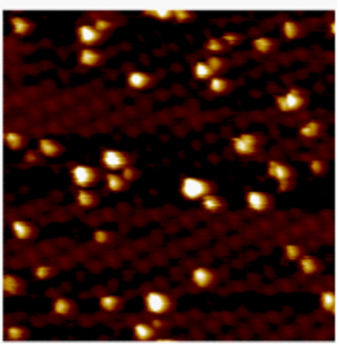

(d) $\mathrm{Rh} / \mathrm{G1} / \mathrm{Ru}(0001)$

Fig. 20. (a) $0.03 \mathrm{ML}$ Ir on $\mathrm{G} 1 / \mathrm{Ir}(111)$ at $350 \mathrm{~K}$ with $\mathrm{FF} \approx 0.65$. Image size: $55 \times 55 \mathrm{~nm}^{2}$. Adapted from Ref. [49] Copyright @ 2006 American Physical Society. (b) 0.05 ML Ru on $\mathrm{G} 1 / \mathrm{Ru}(0001)$ at $305 \mathrm{~K}$ with $\mathrm{FF} \approx 0.29$. Image size: $35 \times 35 \mathrm{~nm}^{2}$.Adapted from Ref. [139] Copyright@2013 American Institute of Physics. (c) $0.05 \mathrm{ML} \mathrm{Pt}$ on G1/Ru(0001) at $309 \mathrm{~K}$ with FF $\approx 0.20$. Image size: $35 \times 35 \mathrm{~nm}^{2}$. Adapted from Ref. [139] Copyright @ 2013 American Institute of Physics. (d) $0.05 \mathrm{ML}$ Rh on G1/Ru(0001) at $295 \mathrm{~K}$ with $\mathrm{FF} \approx 0.13$. Image size: $50 \times 50 \mathrm{~nm}^{2}$. Adapted from Ref. [132] Copyright @ 2010 Elsevier B.V. All 
rights reserved. Note that more complete periodic NC arrays are formed for higher coverages in these systems.
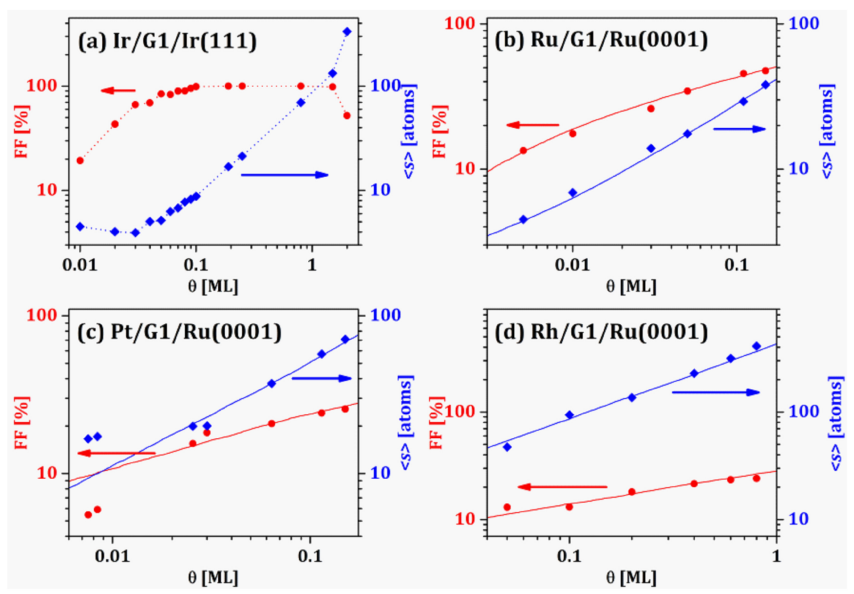

Fig. 21. (a) Ir on $\mathrm{G} 1 / \mathrm{Ir}(111)$ at $350 \mathrm{~K}$ with $\mathrm{F} \approx 0.2 \mathrm{ML} / \mathrm{min}$. The $\theta \approx 2 \mathrm{ML}$ data point reflects coalescence effects. Adapted from Ref. [49] Copyright @ 2006 American Physical Society. (b) $\mathrm{Ru}$ on $\mathrm{G} 1 / \mathrm{Ru}(0001)$ at $305 \mathrm{~K}$ with $\mathrm{F} \approx 0.01-0.1 \mathrm{ML} / \mathrm{min}$. Adapted from Ref. [135] Copyright @ 2013 Materials Research Society. (c) Pt on G1/Ru(0001) at $309 \mathrm{~K}$ with $\mathrm{F} \approx 0.03 \mathrm{ML} / \mathrm{min}$. Adapted from Ref. [135] Copyright @ 2013 Materials Research Society. (d) $\mathrm{Rh}$ on $\mathrm{G} 1 / \mathrm{Ru}(0001)$ at $295 \mathrm{~K}$ simulated for $\mathrm{F} \approx 0.03 \mathrm{ML} / \mathrm{min}$. Adapted from Ref. [132] Copyright @ 2010 Elsevier B.V. All rights reserved. Experimental data is shown by symbols. Smooth curves for (b-d) are produced by our KMC modeling.
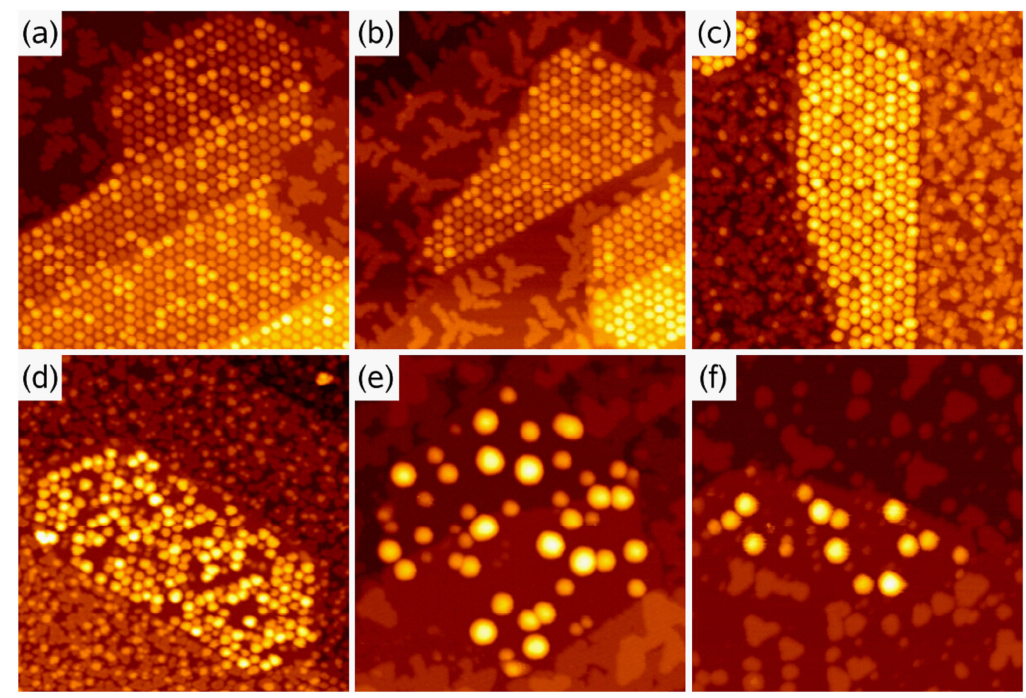

Fig. 22. STM images of Ir, Pt, W, Re, Fe, Au on graphene at room temperature. (a) Ir/G1/Ir(111), 0.20ML. (b) Pt/G1/Ir(111), 0.25ML. (c) W/G1/Ir(111), 0.44ML. (d) Re/G1/Ir(111), 0.53ML. (e) Fe/G1/Ir(111), 0.77ML. (f) Au/G1/Ir(111), 0.25ML. Adapted from Ref. [105]. Copyright @ IOP Publishing Ltd and Deutsche Physikalische Gesellschaft. 


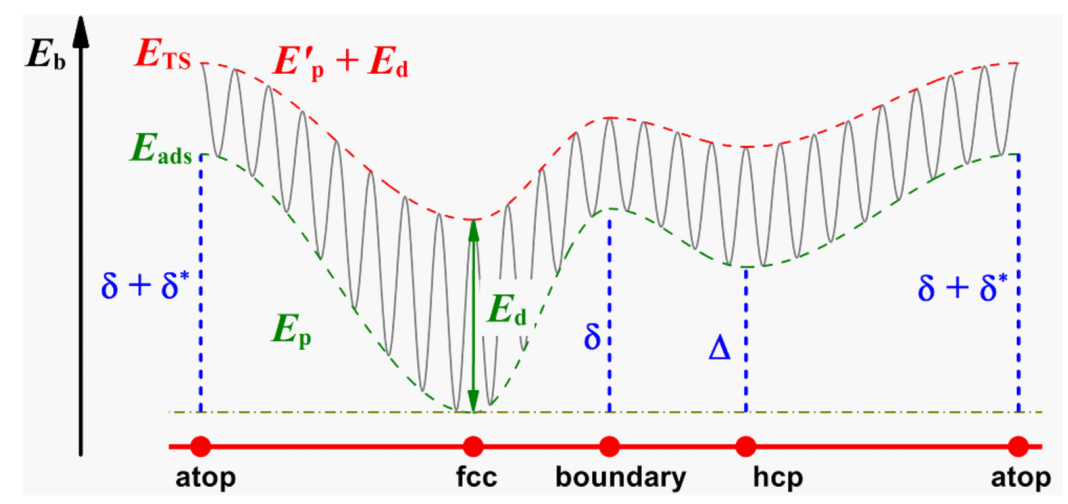

Fig. 23. 1D schematic of the binding energy variation for metal adatoms across the moiré cell. Fine-scale variation of the binding energy, $E_{b}$ : highly oscillatory thin curve where local minima are adsorption sites. Coarse variation of the adsorption energy (green dashed curve) $E_{\text {ads }}=\delta \sin ^{2}\left(\pi \sqrt{3} x / L_{\mathrm{M}}\right)+\delta^{*} \sin ^{2}\left(\pi y / L_{\mathrm{M}}\right)$ in the triangle corresponding to $1 / 6$ of the fcc half moiré cell with $x$ - and $y$-axes shown in Fig. 19(b), and by $E_{\text {ads }}=\Delta+(\delta-\Delta) \sin ^{2}\left(\pi \sqrt{3} x / L_{\mathrm{M}}\right)+\delta^{*} \sin ^{2}\left(\pi y / L_{\mathrm{M}}\right)$ in $1 / 6$ of the hep half moiré cell. Coarse variation of TS energy, $E_{\mathrm{TS}}$ (red dashed curve) with form analogous to $\mathrm{E}_{\text {ads. }}$ Modeling sets $\mathrm{E}_{\mathrm{p}}^{\prime}=\mathrm{E}_{\mathrm{p}}$ with $\Delta=\delta$ and $\delta^{*}=0$, so $\mathrm{E}_{\mathrm{ads}}$ and $\mathrm{E}_{\mathrm{TS}}$ dashed curves are flat throughout the hcp region. Adapted from Ref. [139]. Copyright @ 2013 American Institute of Physics. 

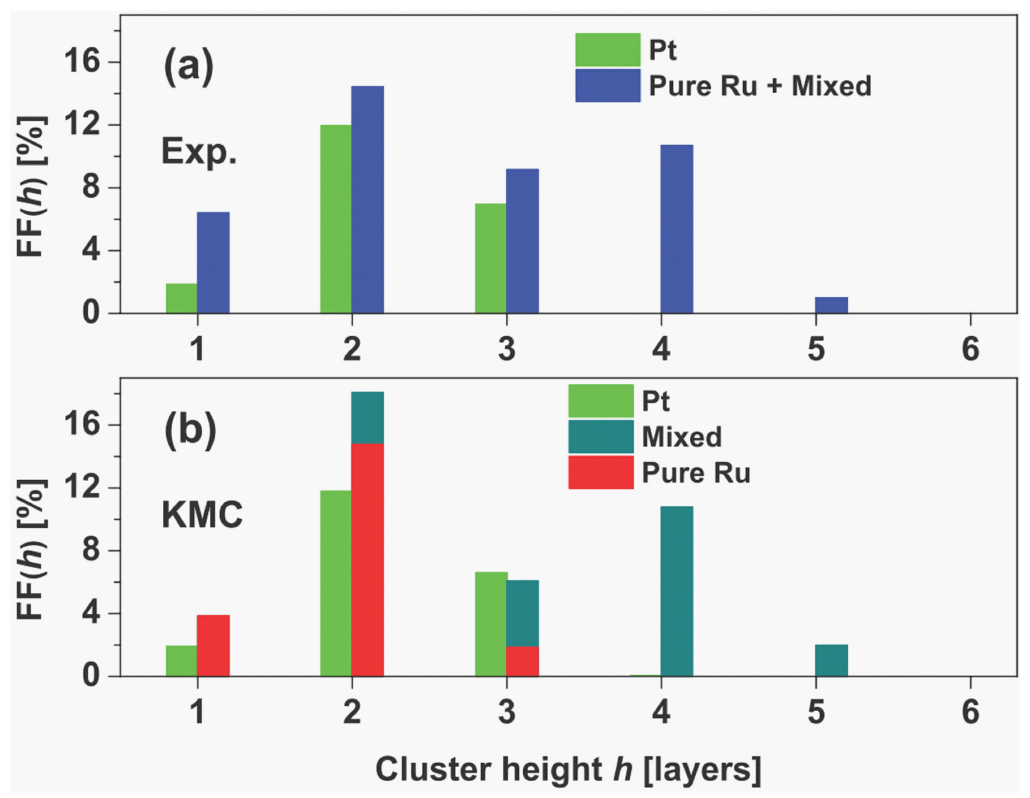

Fig. 24. NC height distributions FF(h): (a) Experimental height distribution after Pt deposition and after Ru@Pt deposition. (b) Corresponding KMC results where in bars for $\mathrm{Ru} @ \mathrm{Pt}$ indicate separate contributions from mixed and pure Ru NCs. Here X@Y means Y then X. Adapted from Ref. [139]. Copyright @ 2013 American Institute of Physics.
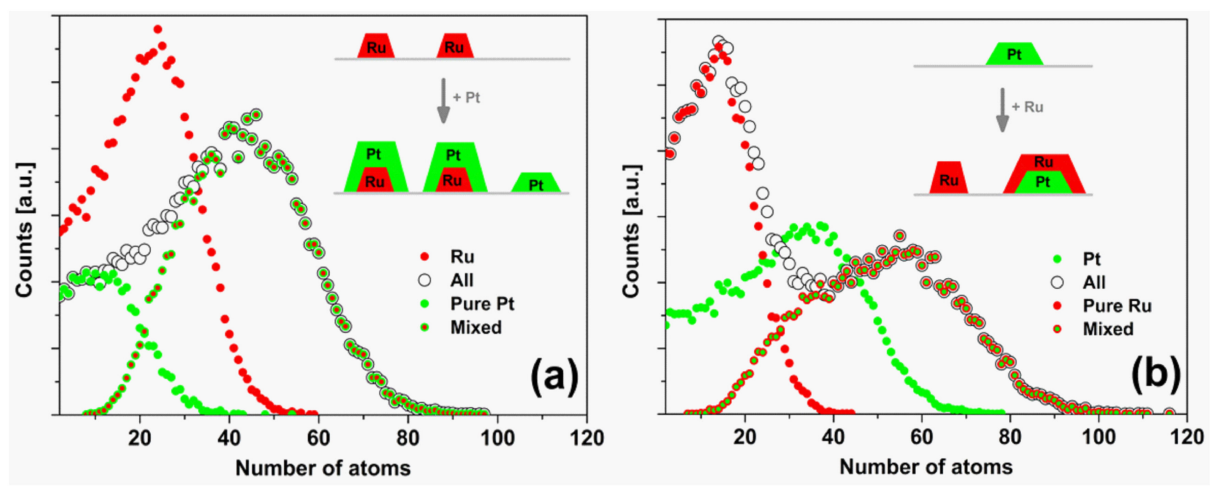

Fig. 25. Predictions for size distributions for various types of NCs following sequential co-deposition of Pt and Ru on G1/Ru(0001) at 300-305 K. (a) 0.05 ML Ru then 0.06 ML Pt. (b) 0.05 ML Pt then 0.06 ML Ru. Adapted from Ref. [139]. Copyright @ 2013 American Institute of Physics. 


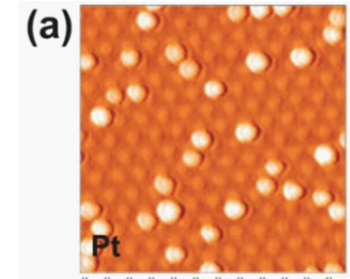

(c)

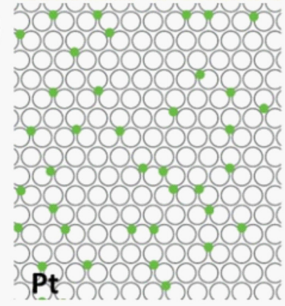

(b)

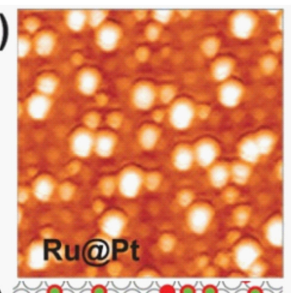

(d)

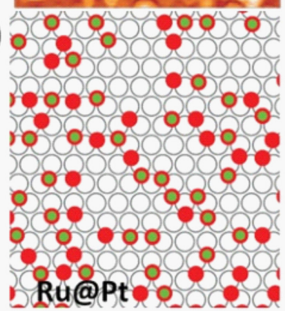

Fig. 26. STM images of NC distributions for Pt deposition, (a), followed by Ru deposition $\mathrm{Ru} @ \mathrm{Pt}$, (b), under conditions described in the text. Image size: $35 \times 35 \mathrm{~nm}^{2}$. KMC simulation of NC distributions for Pt deposition, (c), followed by Ru deposition Ru @Pt, (d), under conditions described in the text. Image size: $89 \times 53 \mathrm{~nm}^{2}$. Pure Pt $(\mathrm{Ru}) \mathrm{NCs}$ are green (red), and mixed NCs have a green core and red ring. Adapted from Ref. [139]. Copyright@2013 American Institute of Physics.
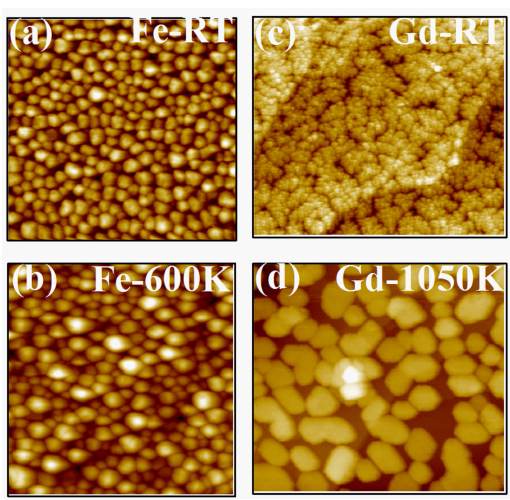
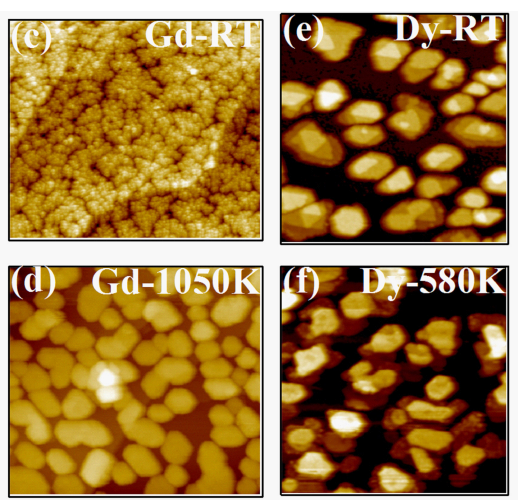
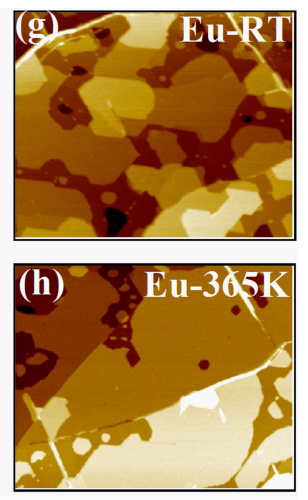

Fig.27. (a) Fe/Graphene: $250 \times 250 \mathrm{~nm}^{2}$, RT, $3 \mathrm{ML}, \mathrm{F}=0.25 \mathrm{ML} / \mathrm{min}, \mathrm{N}=5.1 \times 10^{-3}$ $\mathrm{NCs} / \mathrm{nm}^{2}$; (b) Annealing at $600 \mathrm{~K}$ for $20 \mathrm{~min}$ from (a) does not change NC density significantly, only the shape of individual NCs equilibrates and their average height increases; (c) Gd/Graphene: $250 \times 215 \mathrm{~nm}^{2}$, RT, 4ML, F = 0.1ML/min; (d) Fractal morphology is stable up to $800 \mathrm{~K}$, but transition to crystalline shapes occurs at higher temperature, as shown in (d) where previous film was annealed at $1050 \mathrm{~K}$ for $5 \mathrm{~min}$; (e) Dy/Graphene: $100 \times 100 \mathrm{~nm}^{2}$, RT, $2.0 \mathrm{ML}, 0.22 \mathrm{ML} / \mathrm{min}, \mathrm{N}=6 \times 10^{-3} \mathrm{NCs} / \mathrm{nm}^{2}$; (f) annealing of (e) to $580 \mathrm{~K}$ transforms multiple level NCs with 4-7 Dy layers exposed into more uniform height shaped NCs and some of them coalesce, indicating lower thermal stability of Dy nanoparticles on graphene, as compared with Fe and Gd; (g) Eu/Graphene: $250 \times 200 \mathrm{~nm}^{2}$, RT, 2.6ML, $0.16 \mathrm{ML} / \mathrm{min}$; (h) Annealing of the Eu film from (g) at $365 \mathrm{~K}$ for 15 min transforms the lager islands into almost continuous film with thickness of 3 ML. Adapted from Ref. [90]. Copyright @ 2012 American Physical Society. 

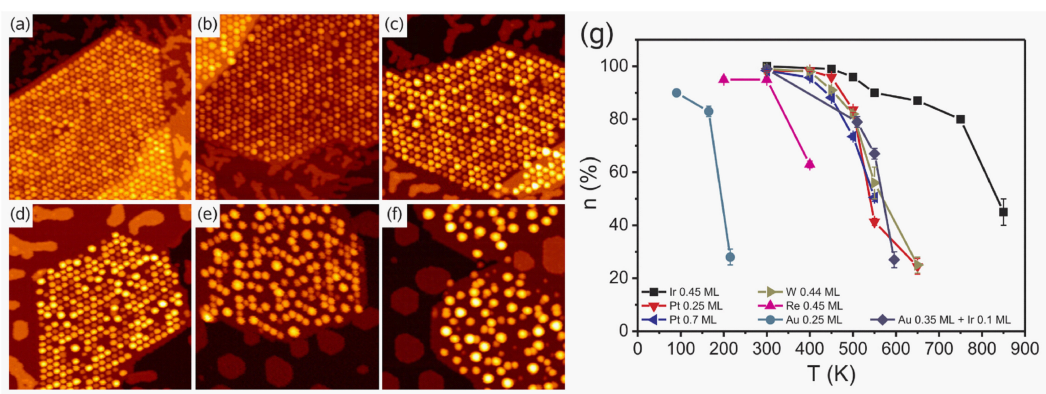

Fig. 28. Annealing STM images (i.e., $70 \times 70 \mathrm{~nm}^{2}$ ) of Pt cluster superlattice on graphene on $\operatorname{Ir}(111)$ with $0.25 \mathrm{ML}$ at (a) $300 \mathrm{~K}$. (b) $400 \mathrm{~K}$. (c) $450 \mathrm{~K}$. (d) $500 \mathrm{~K}$. (e) $550 \mathrm{~K}$. (f) $650 \mathrm{~K}$. (g) Occupation probability $\mathrm{n}$ (or filling fraction, FF) of moiré unit cell $\mathrm{s}$ with clusters as a function of annealing temperature T. (h) Arrhenius plot of cluster hopping rate $v(\mathrm{~T})$. Adapted from Ref. [105]. Copyright @ IOP Publishing Ltd and Deutsche Physikalische Gesellschaft. 

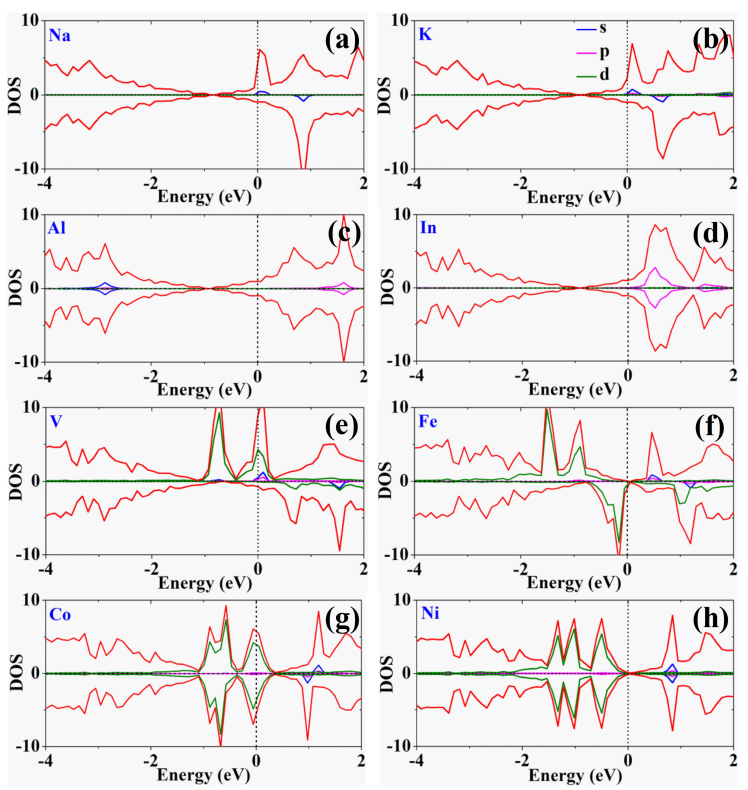

FIG. 29. Spin polarized electronic density of states (DOS) of adatom-graphene systems. The two spin polarizations are shown above and below the horizontal axis. The projected local partial DOS of the adatoms with the $s$ (blue thick solid line), $p$ (pink dashed line), and $d$ (green dash-dot line) characters respectively are also shown. Adapted from Ref. [57]. Copyright@2011 American Physical Society.
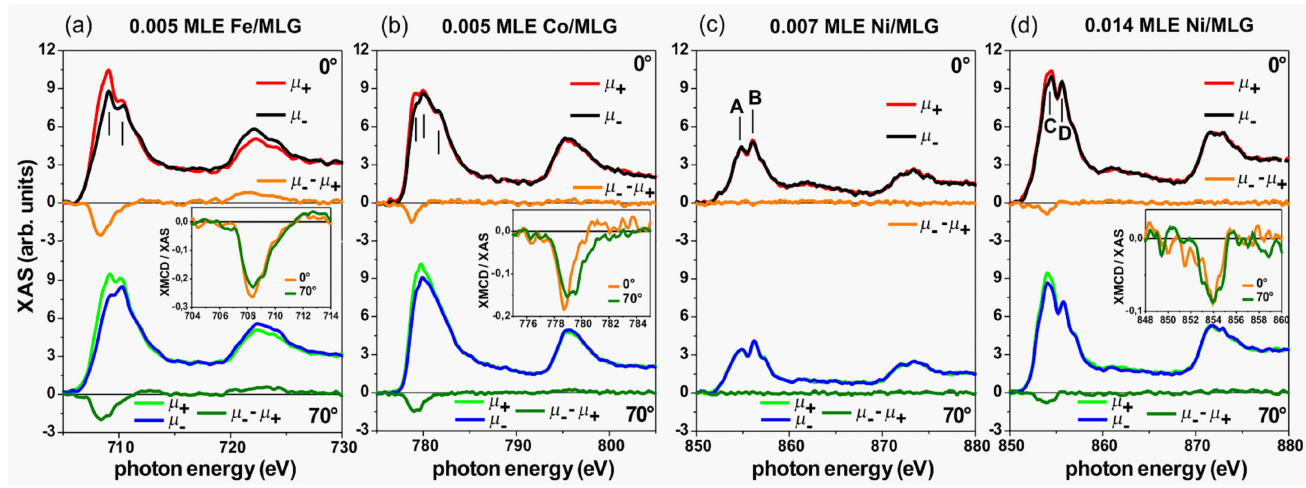

Fig. 30. XAS and XMCD measurements for (a) Fe (0.005 monolayer equivalent (MLE)), (b) Co (0.005 MLE), (c) Ni adatoms (0.007 MLE), and (d) higher Ni coverage (0.014 MLE) at $\mathrm{T}=10 \mathrm{~K}, \mathrm{~B}=5 \mathrm{~T}$. The upper and lower panels display the $\mathrm{L}_{3,2}$ edges of XAS and XMCD spectra achieved with parallel $\left(_{+}\right)$and antiparallel (.) alignment between the helicity of the incident beam and the magnetic field B, obtained at normal (0) and grazing (70) incident angle. The spectra have been normalized with respect to the incident beam intensity and the $\mathrm{L}_{3}$ preedge intensity. The insets in (a), (b), and (d) show the XMCD divided by the averaged XAS intensity for 0 and 70, respectively. Adapted from Ref. [84]. Copyright @ 2013 American Physical Society. 


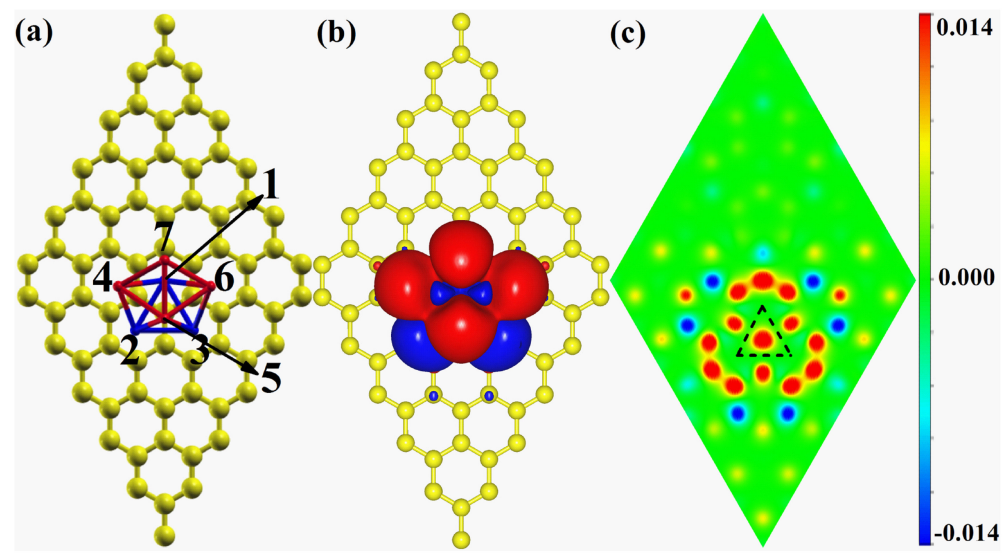

Fig. 31. Spin-polarized electron density difference (i.e., $\left.\Delta \rho(r)=\rho_{u p}(r)-\rho_{d w}(r)\right)$ of $M n_{7}$ on graphene. (a) The most stable geometry of $\mathrm{Mn}_{7}$ cluster on graphene with $\mathrm{Mn}$ atom number labeled. (b) 3D spin-polarized $\Delta \rho(\mathrm{r})$ of $\mathrm{Mn}_{7}$ cluster on graphene. (c) 2D contour plot of spin-polarized $\Delta \rho(r)$ on the graphene layer. Dashed triangle represents the position of the first three Mn atoms. Red color indicates spin-up electron density, and blue color indicates spin-down electron density. Adapted from Ref. [176]. Copyright @ 2014 American Chemical Society.
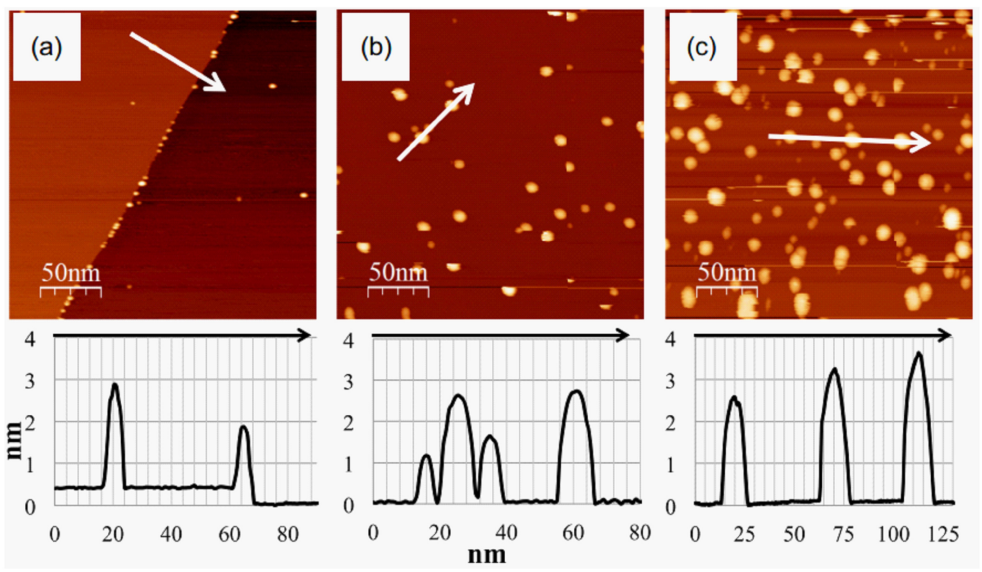

Fig. 32. (Color online) STM images and line profiles of $\mathrm{Cu}$ clusters on HOPG terraces, 250 $\times 250 \mathrm{~nm}^{2},-0.8$ to $-1.0 \mathrm{~V}, 0.1 \mathrm{nA}$. (a) $0.003 \mathrm{ML}$, (b) $0.10 \mathrm{ML}$, and (c) $0.21 \mathrm{ML}$. Adapted from Ref . [185]. Copyright @ 2014 American Physical Society. 

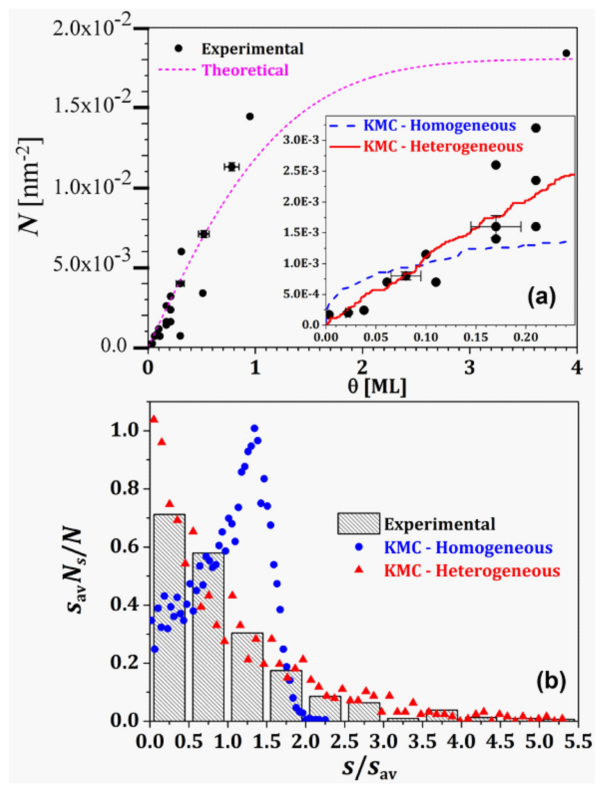

Fig. 33. (Color online) (a) $\mathrm{N}$ vs coverage $(\theta)$ for experiment (dots), and refined Johnson-Mehl-Avrami-Kolmogorov theory (dashed line). Inset: KMC results for homogeneous (dashed line) and heterogeneous (solid line) nucleation. Error bars reflect statistical uncertainty based on sample size. Ion flux variation is reflected in scatter. (b) Scaled island size distribution for 0.1 ML from experiment (bars, 632 islands total), and from KMC simulations for homogeneous nucleation (blue circles, 5164 islands) and heterogeneous nucleation (red triangles, 1261 islands). Adapted from Ref. [185]. Copyright @ 2014 American Physical Society. 